\title{
Ynamide-Mediated Intermolecular Esterification
}

\author{
Xuewei Wang ${ }^{\dagger \S}$, Yang Yang ${ }^{\dagger \S}$, Yongli Zhao ${ }^{\dagger}$, Sheng Wang ${ }^{\dagger}$, Wenchang $\mathrm{Hu}^{\dagger}$, Jinmei \\ $\mathrm{Li}^{\dagger}$, Zihao Wang ${ }^{\dagger}$, Fengling Yang ${ }^{\star *}$, Junfeng Zhao ${ }^{\dagger *}$ \\ ${ }^{\dagger}$ College of Chemistry and Chemical Engineering, Jiangxi Normal University, Nanchang, 330022, P. R. \\ China \\ "Henan University of Urban Construction, Pingdingshan, 467036, Henan, P. R. China \\ *E-mail: zhaojf@jxnu.edu.cn; yangfl@hncj.edu.cn \\ ${ }^{\S}$ Xuewei Wang and Yang Yang contributed equally.
}

\section{Table of Contents}

NMR Spectra 


\section{NMR Spectra}

${ }^{1} \mathrm{H}-\mathrm{NMR}\left(400 \mathrm{MHz}\right.$ ) spectrum of compound 3aa in $\mathrm{CDCl}_{3}$.

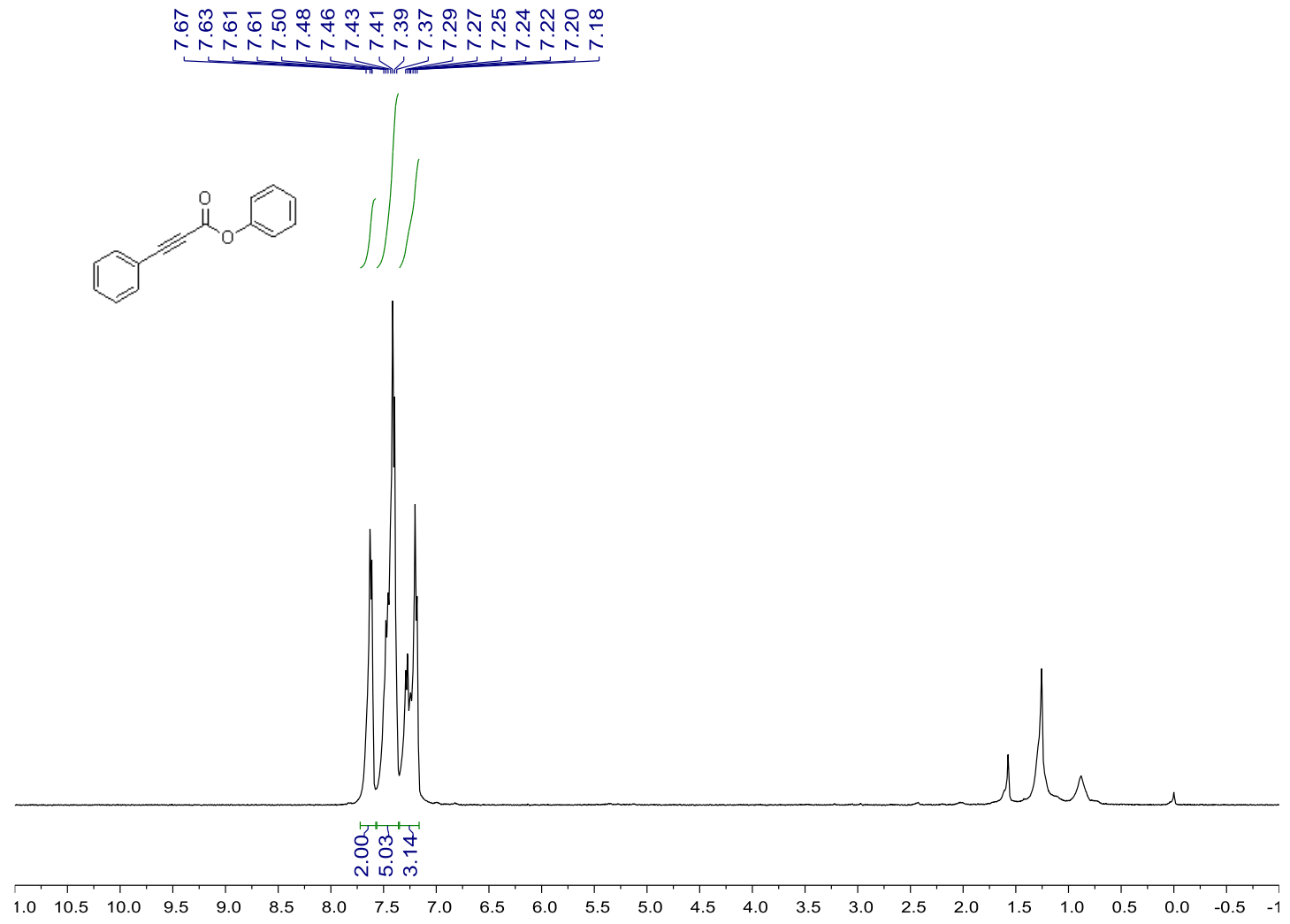

${ }^{13} \mathrm{C}\left\{{ }^{1} \mathrm{H}\right\}$-NMR (100 MHz) spectrum of compound 3aa in $\mathrm{CDCl}_{3}$.

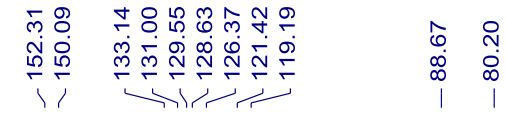
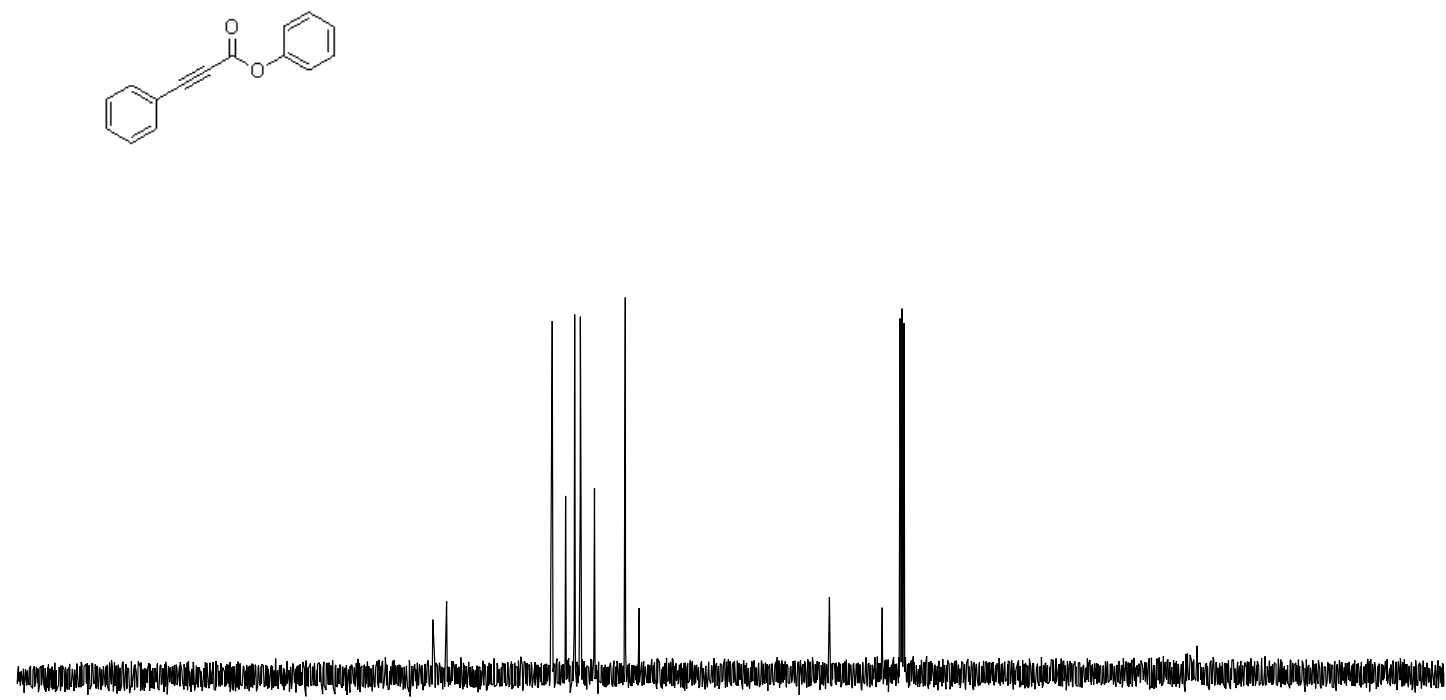

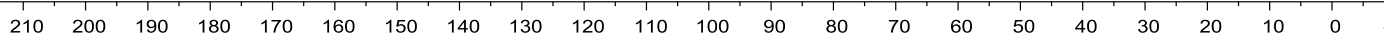


${ }^{1} \mathrm{H}-\mathrm{NMR}(400 \mathrm{MHz})$ spectrum of compound $4 \mathbf{a}$ in $\mathrm{CDCl}_{3}$.

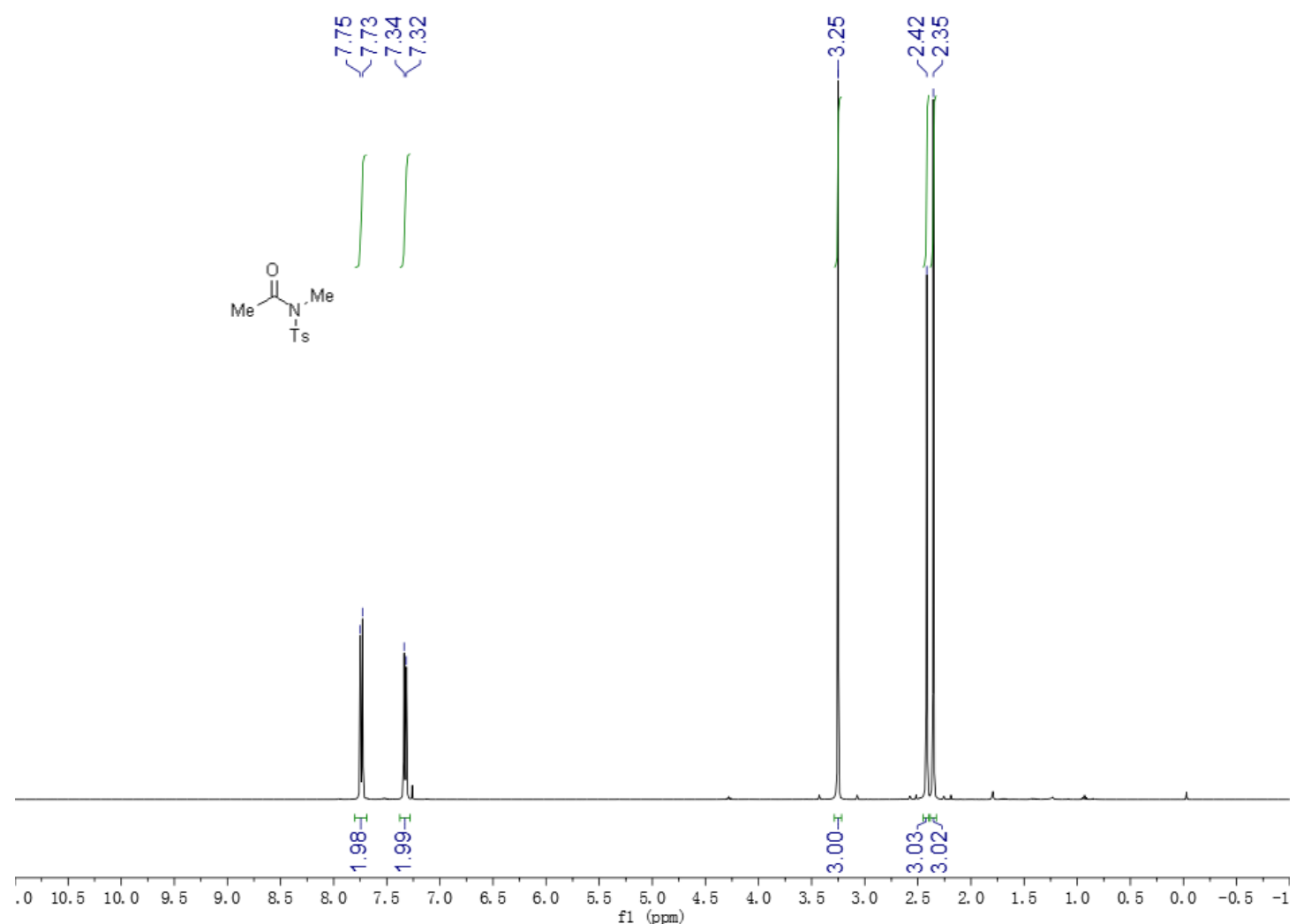

${ }^{13} \mathrm{C}\left\{{ }^{1} \mathrm{H}\right\}$-NMR (100 MHz) spectrum of compound 4a in $\mathrm{CDCl}_{3}$.

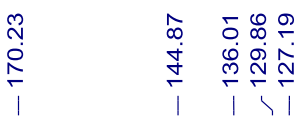

ஓ另字

लึ่ง

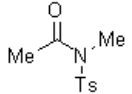
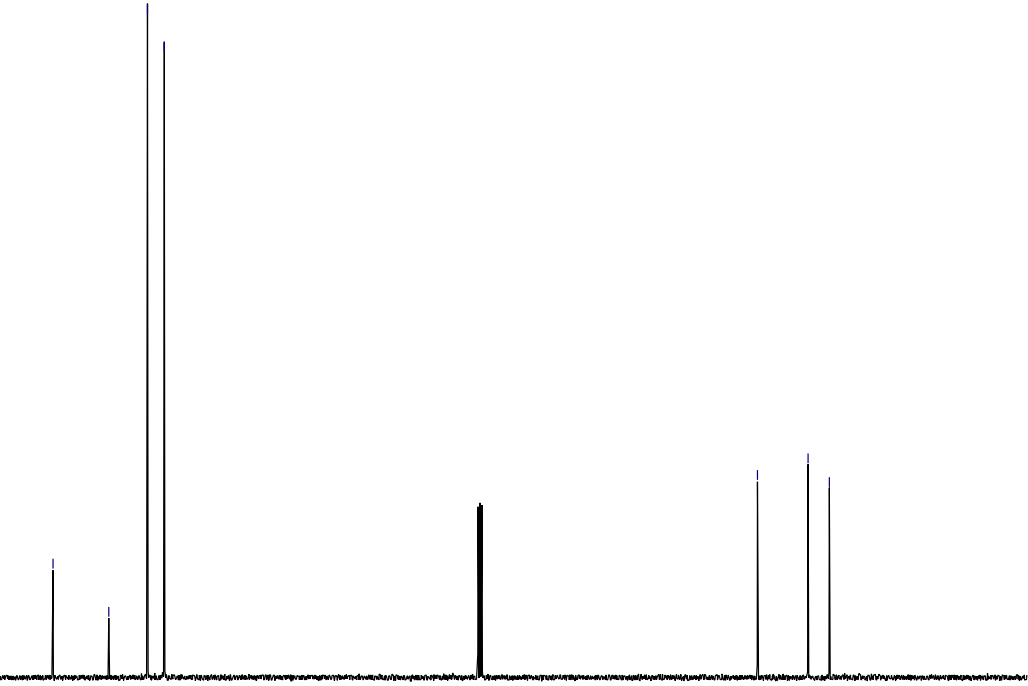

$\begin{array}{rrrrrrrrrrrr}110 & 200 & 190 & 180 & 170 & 160 & 150 & 140 & 130 & 120 & 110 & 100 \\ f 1 & (\mathrm{ppm})\end{array}$ 
${ }^{1} \mathrm{H}-\mathrm{NMR}(400 \mathrm{MHz})$ spectrum of compound 3ab in $\mathrm{CDCl}_{3}$.

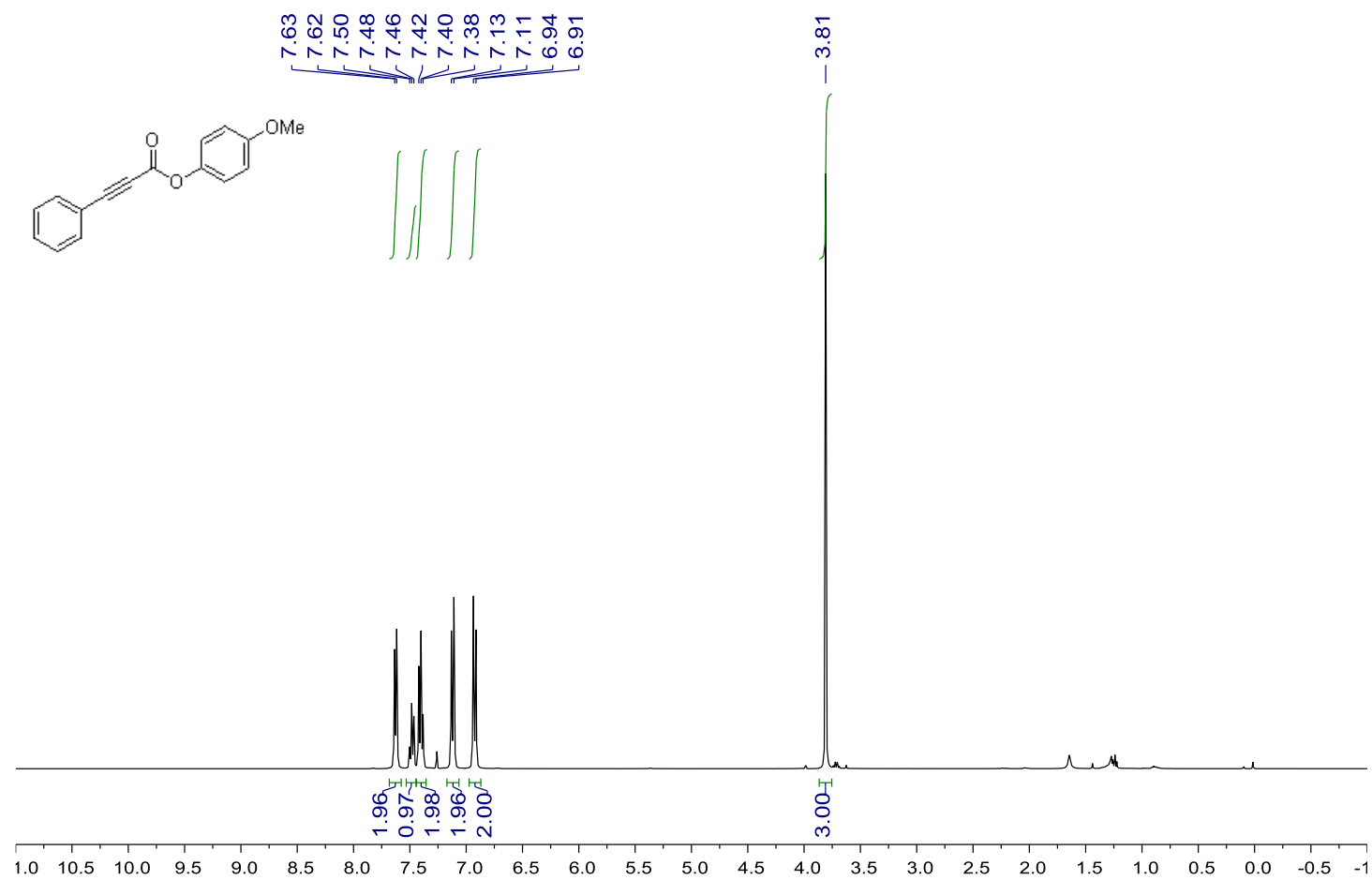

${ }^{13} \mathrm{C}\left\{{ }^{1} \mathrm{H}\right\}$-NMR (100 MHz) spectrum of compound $\mathbf{3 a b}$ in $\mathrm{CDCl}_{3}$.

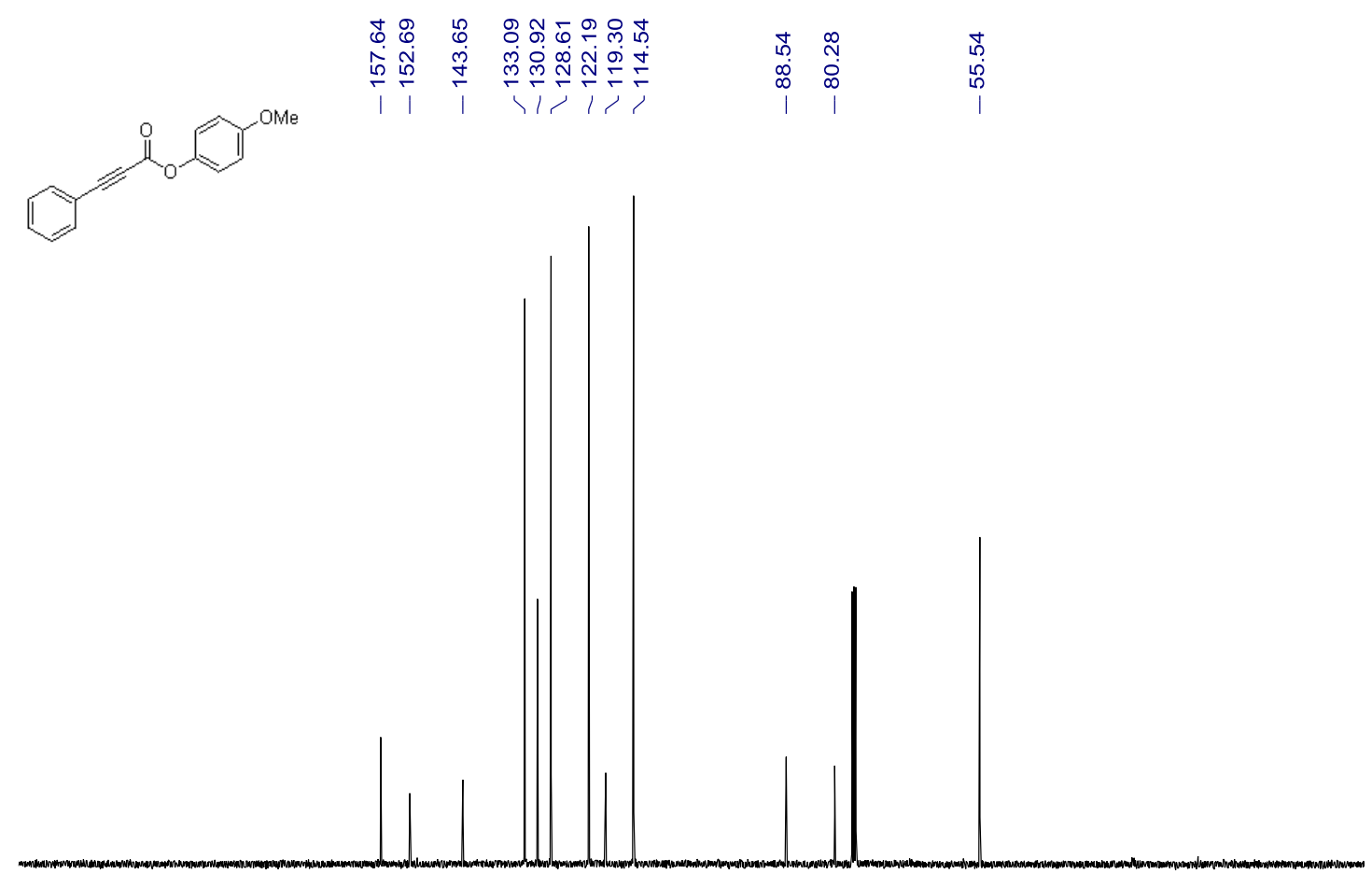

$\begin{array}{llllllllllllllllllllll}210 & 200 & 190 & 180 & 170 & 160 & 150 & 140 & 130 & 120 & 110 & 100 & 90 & 80 & 70 & 60 & 50 & 40 & 30 & 20 & 10 & 0\end{array}$. 
${ }^{1} \mathrm{H}-\mathrm{NMR}\left(400 \mathrm{MHz}\right.$ ) spectrum of compound $3 \mathbf{a c}$ in $\mathrm{CDCl}_{3}$.

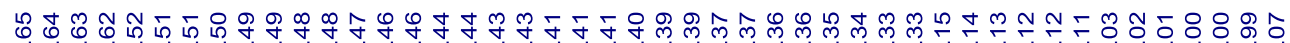

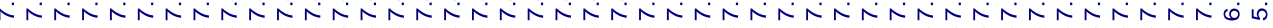
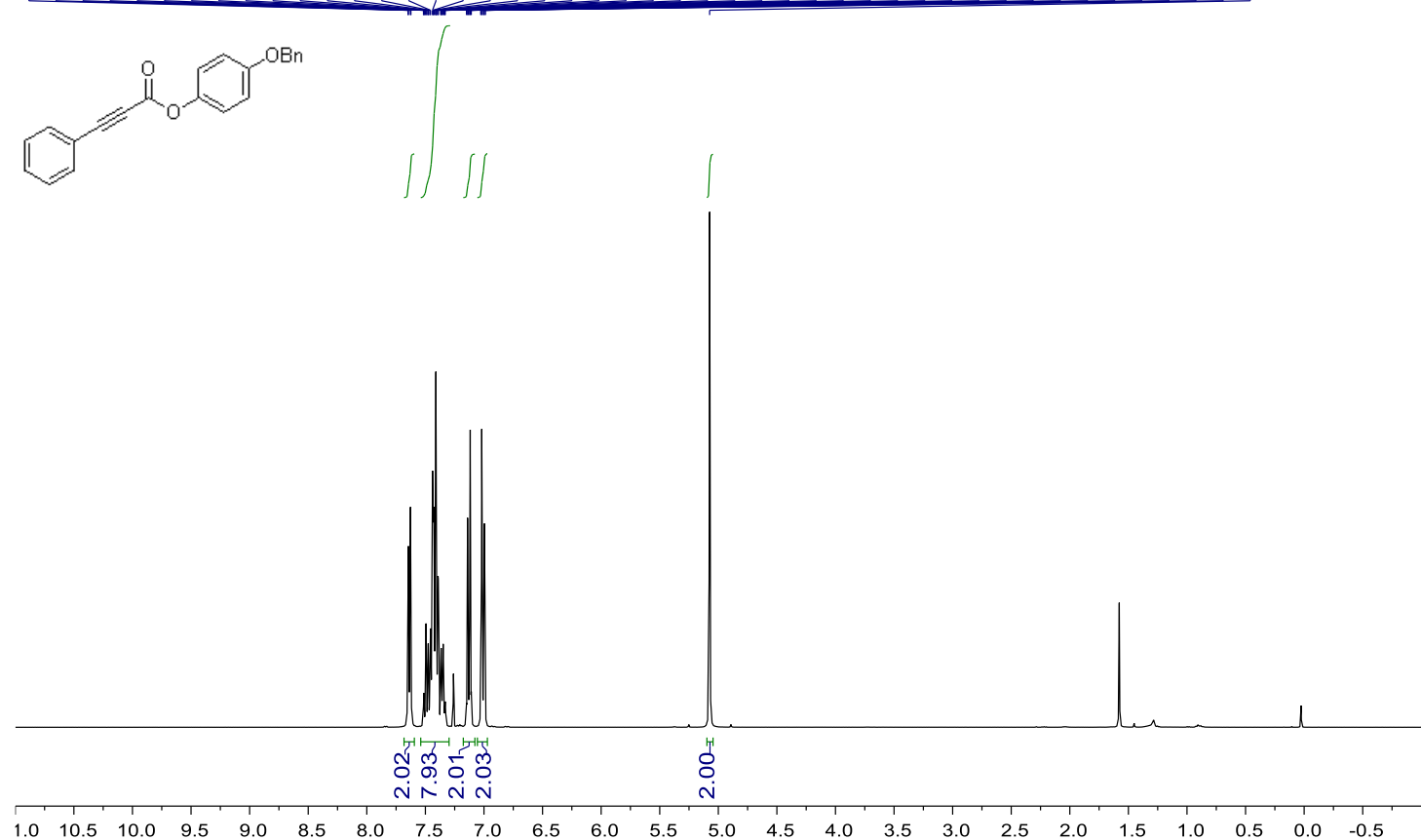

${ }^{13} \mathrm{C}\left\{{ }^{1} \mathrm{H}\right\}$-NMR (100 MHz) spectrum of compound $3 \mathbf{a c}$ in $\mathrm{CDCl}_{3}$

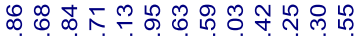
它

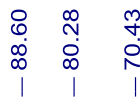<smiles>O=C(Oc1ccc(Br)cc1)C1CC2CCCCC21</smiles>

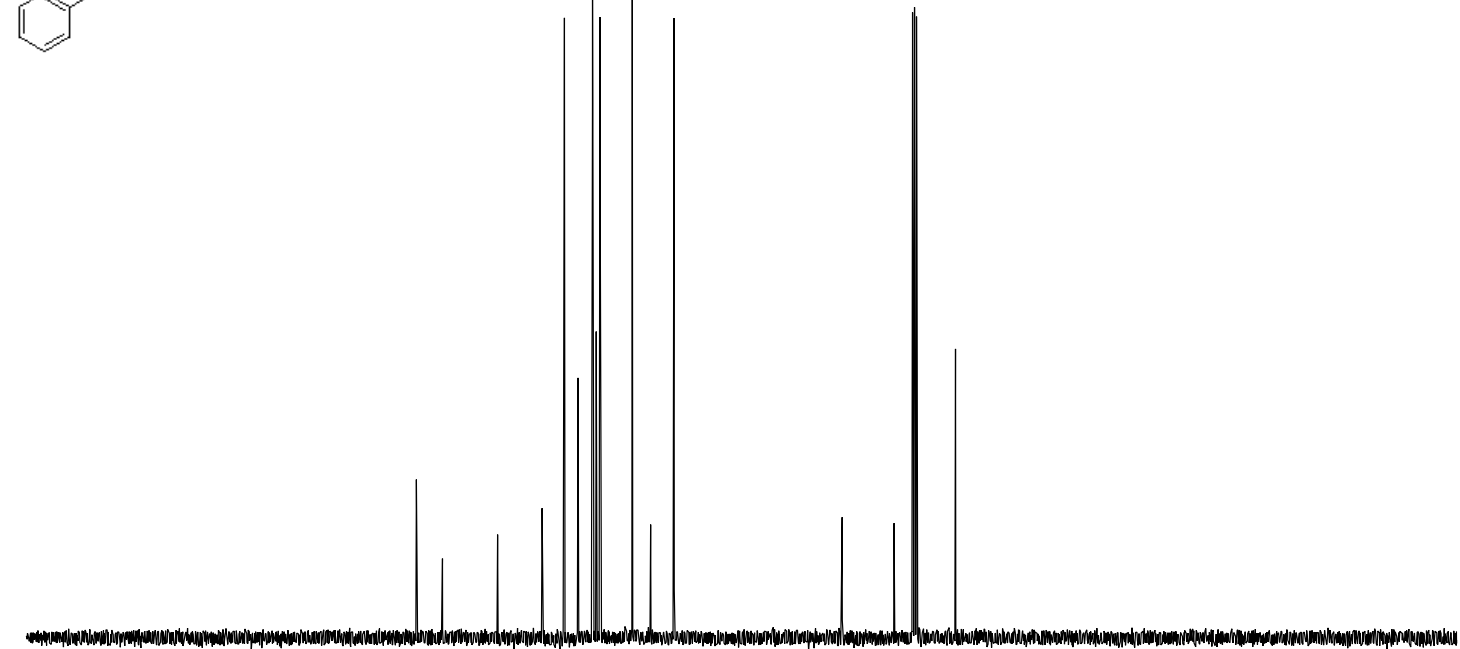

$\begin{array}{llllllllllllllllllllll}210 & 200 & 190 & 180 & 170 & 160 & 150 & 140 & 130 & 120 & 110 & 100 & 90 & 80 & 70 & 60 & 50 & 40 & 30 & 20 & 10 & 0\end{array}$. 
${ }^{1} \mathrm{H}-\mathrm{NMR}(400 \mathrm{MHz})$ spectrum of compound 3ad in $\mathrm{CDCl}_{3}$.

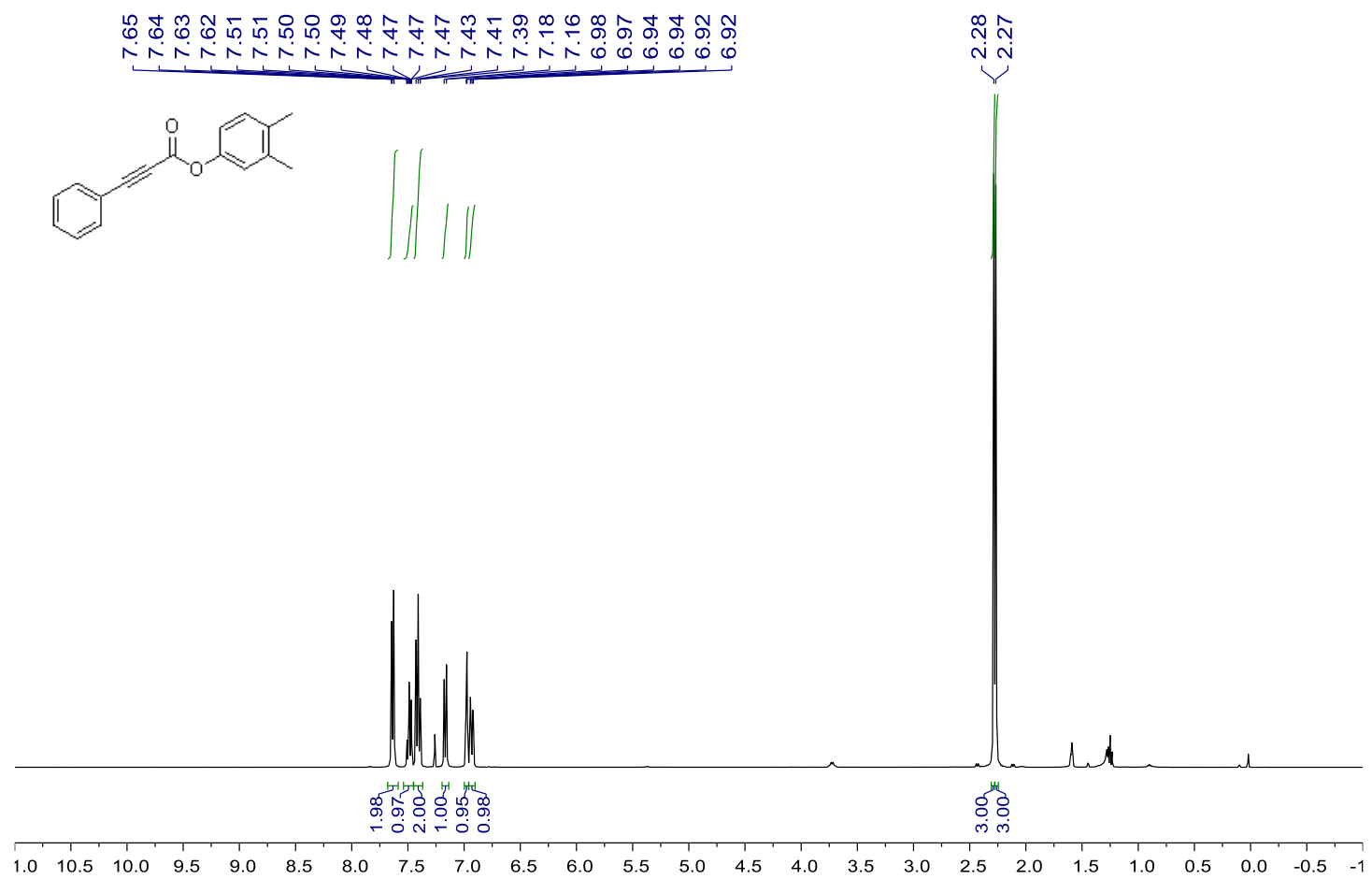

${ }^{13} \mathrm{C}\left\{{ }^{1} \mathrm{H}\right\}$-NMR $(100 \mathrm{MHz})$ spectrum of compound $\mathbf{3 a d}$ in $\mathrm{CDCl}_{3}$

|
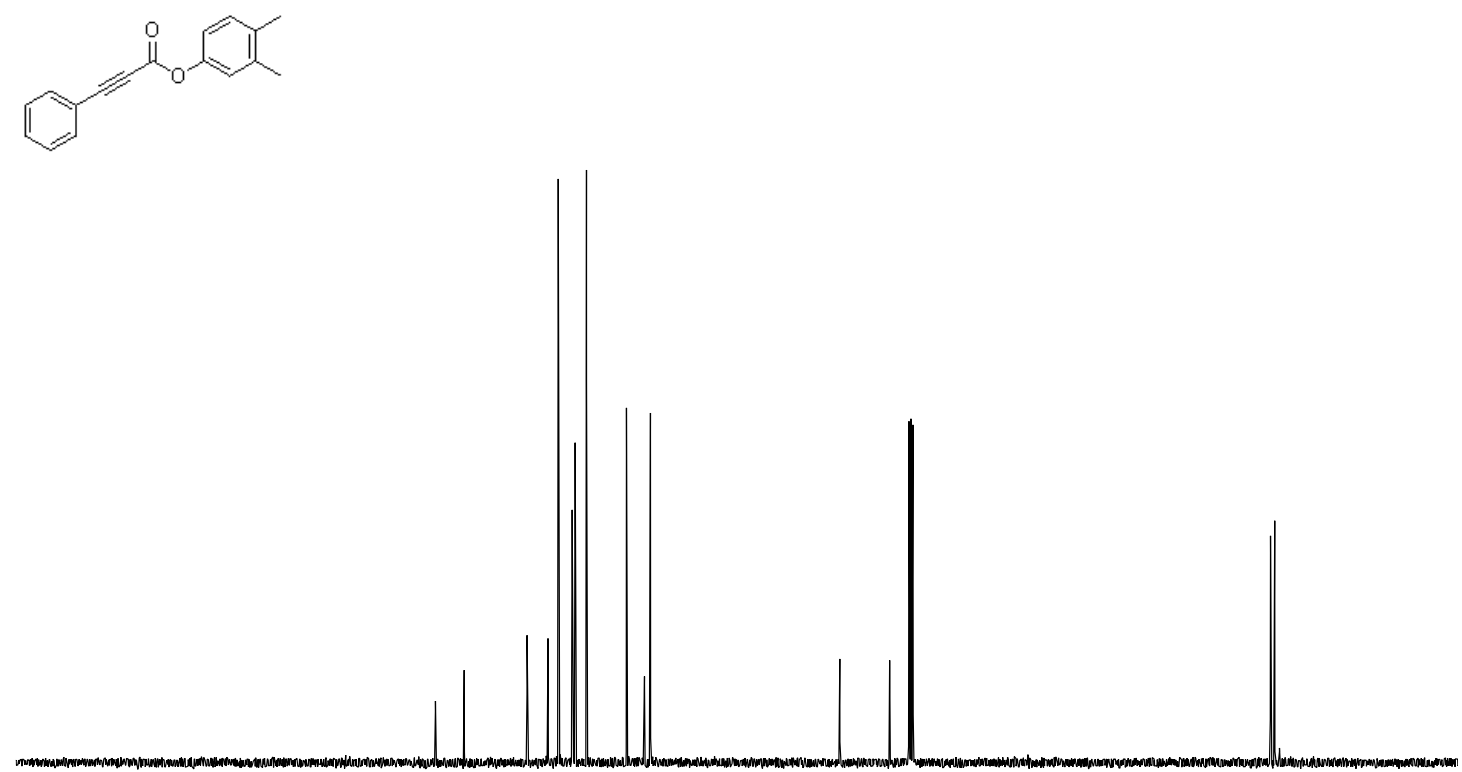

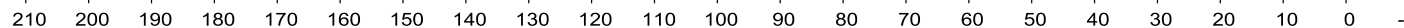


${ }^{1} \mathrm{H}-\mathrm{NMR}(400 \mathrm{MHz})$ spectrum of compound 3ae in $\mathrm{CDCl}_{3}$.

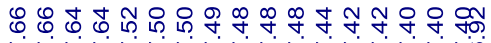

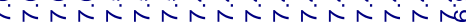
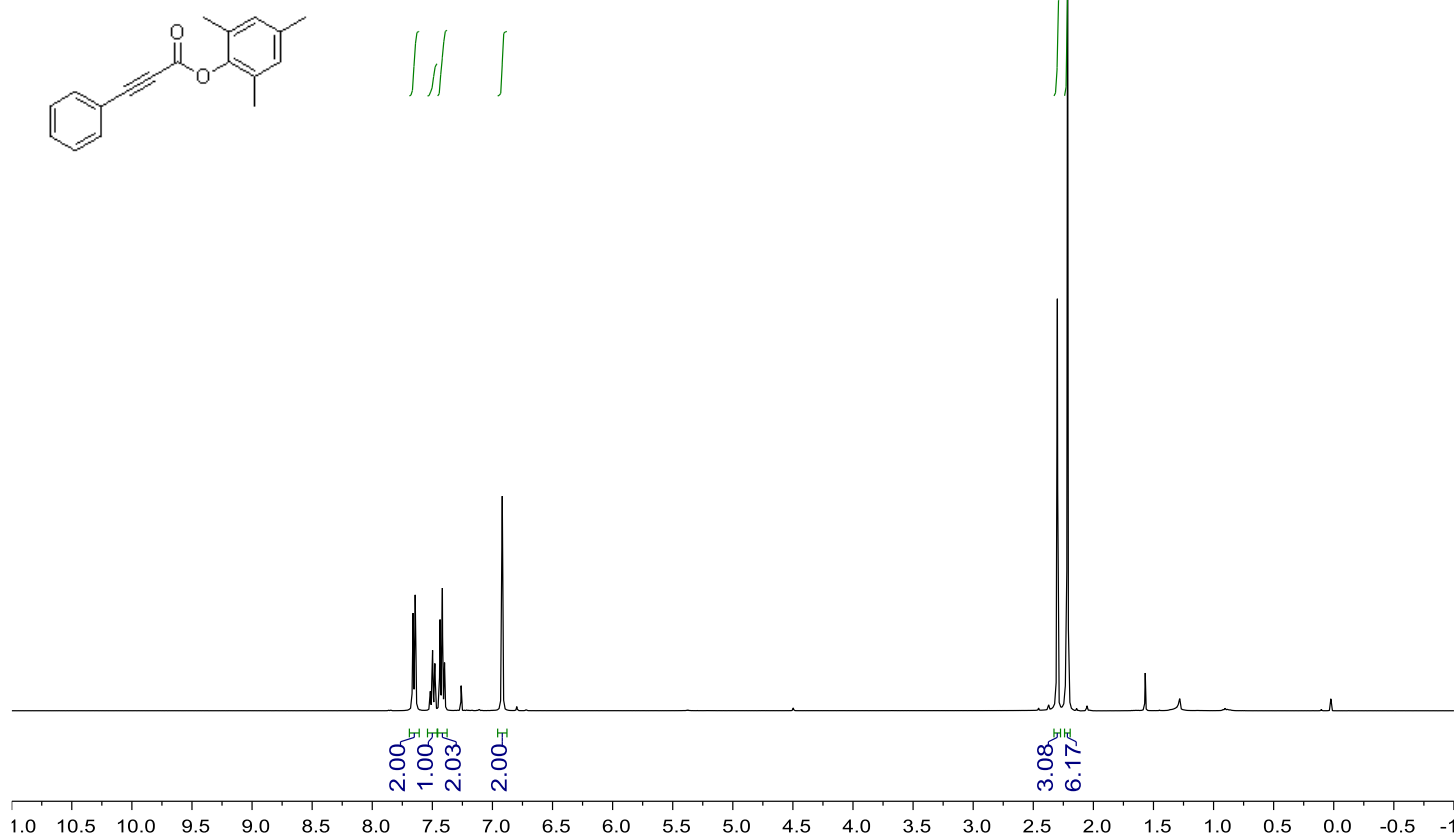

${ }^{13} \mathrm{C}\left\{{ }^{1} \mathrm{H}\right\}$-NMR (100 MHz) spectrum of compound 3ae in $\mathrm{CDCl}_{3}$

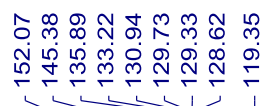

$\begin{array}{cc}\infty & 0 \\ & 0 \\ \infty & 0 \\ 1 & 0\end{array}$

$\cos$

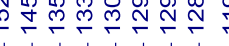

$\stackrel{4}{1} \stackrel{1}{\infty}$
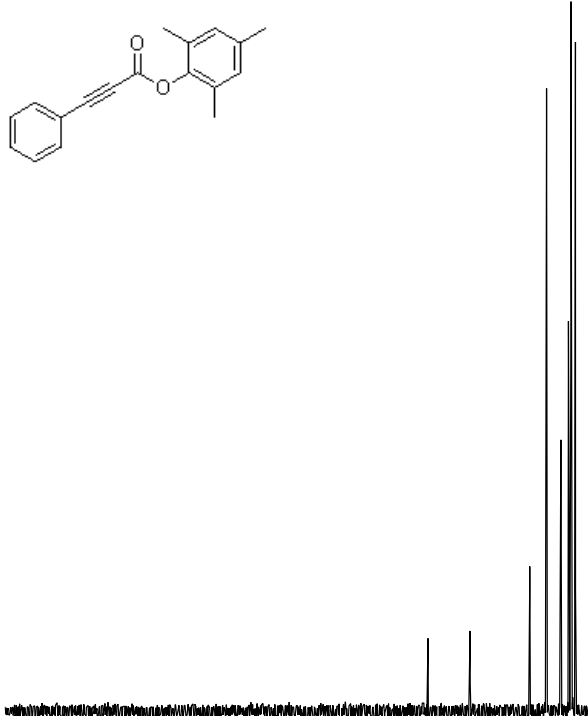

$\begin{array}{llllllllllll}210 & 200 & 190 & 180 & 170 & 160 & 150 & 140 & 130 & 120 & 110 & 100\end{array}$ 
${ }^{1} \mathrm{H}-\mathrm{NMR}(400 \mathrm{MHz})$ spectrum of compound 3af in $\mathrm{CDCl}_{3}$

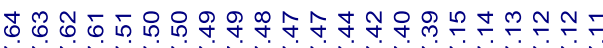

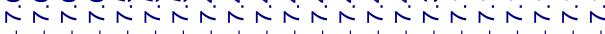
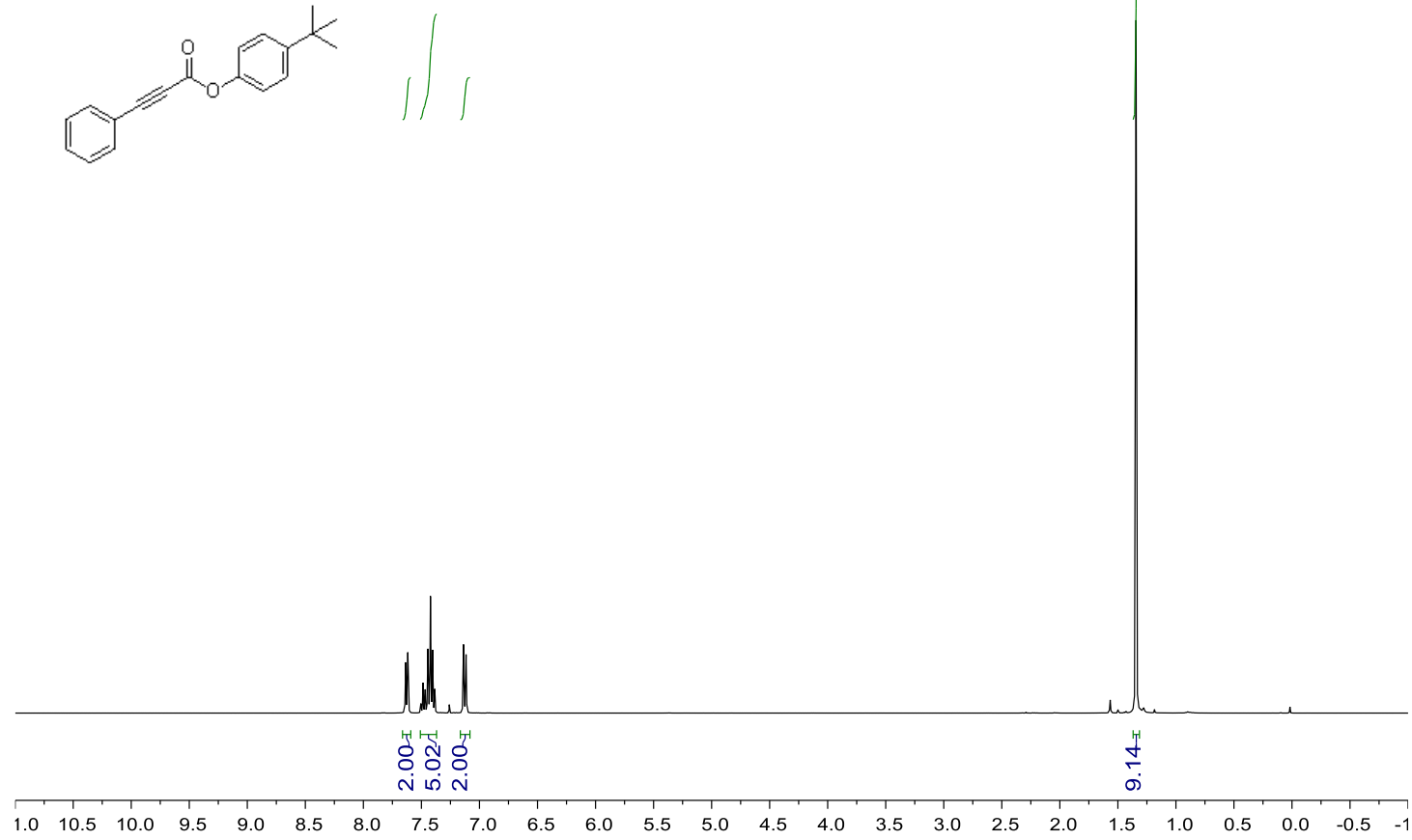

${ }^{13} \mathrm{C}\left\{{ }^{1} \mathrm{H}\right\}$-NMR (100 MHz) spectrum of compound 3 af in $\mathrm{CDCl}_{3}$

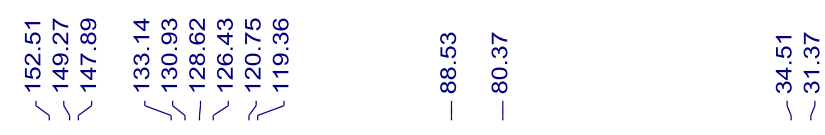
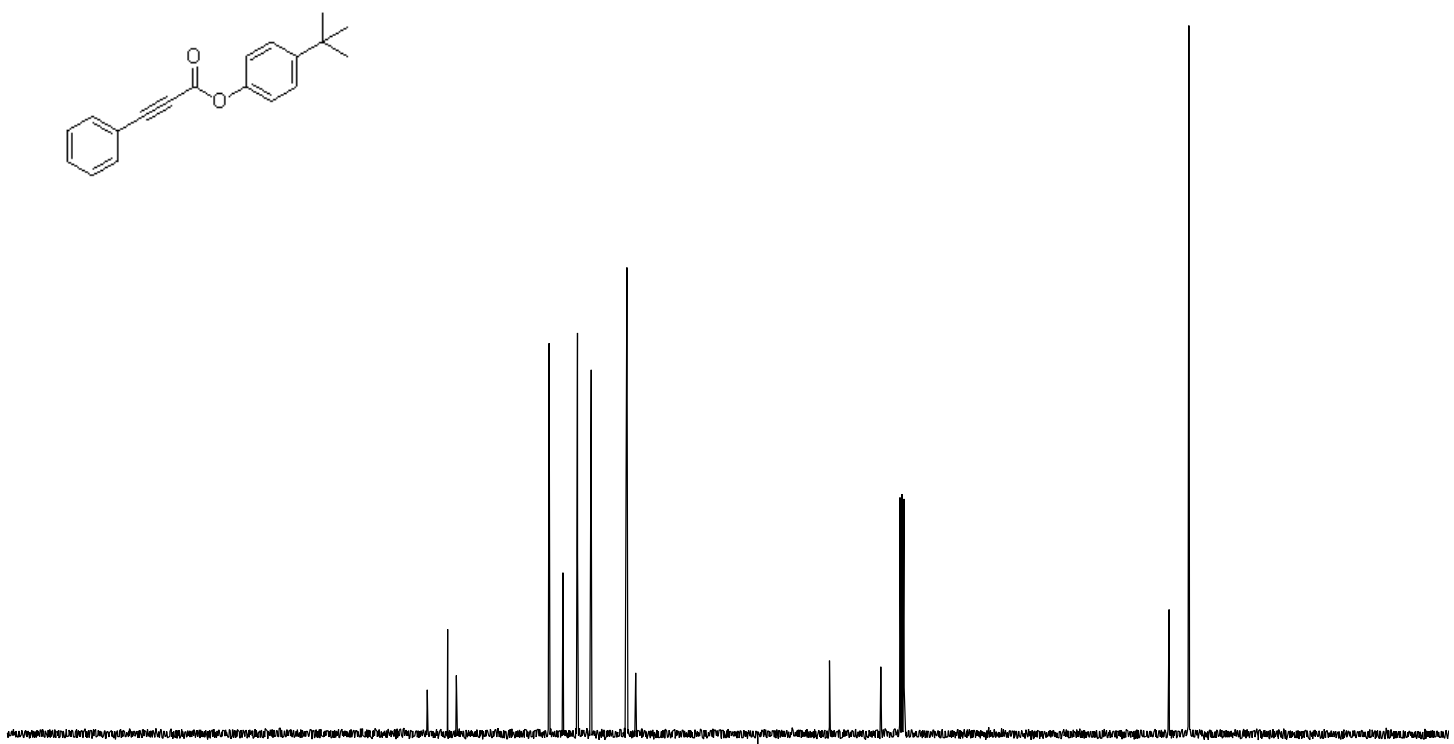

$\begin{array}{llllllllllllllllllllll}210 & 200 & 190 & 180 & 170 & 160 & 150 & 140 & 130 & 120 & 110 & 100 & 90 & 80 & 70 & 60 & 50 & 40 & 30 & 20 & 10 & 0\end{array}$ 
${ }^{1} \mathrm{H}-\mathrm{NMR}$ (400 MHz) spectrum of compound $\mathbf{3 a g}$ in $\mathrm{CDCl}_{3}$
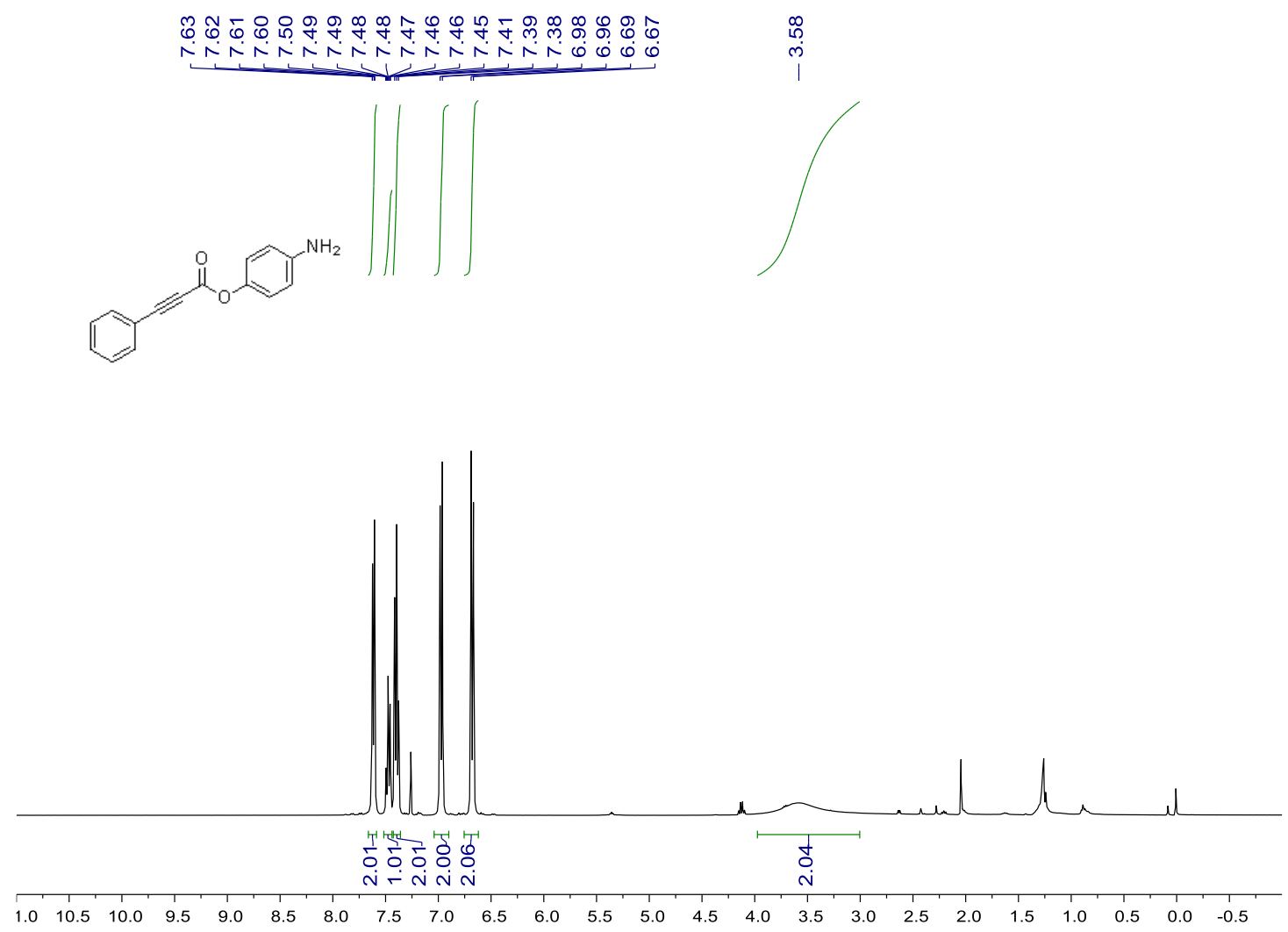

${ }^{13} \mathrm{C}\left\{{ }^{1} \mathrm{H}\right\}$-NMR (100 MHz) spectrum of compound $\mathbf{3 a g}$ in $\mathrm{CDCl}_{3}$

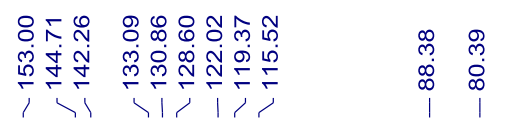
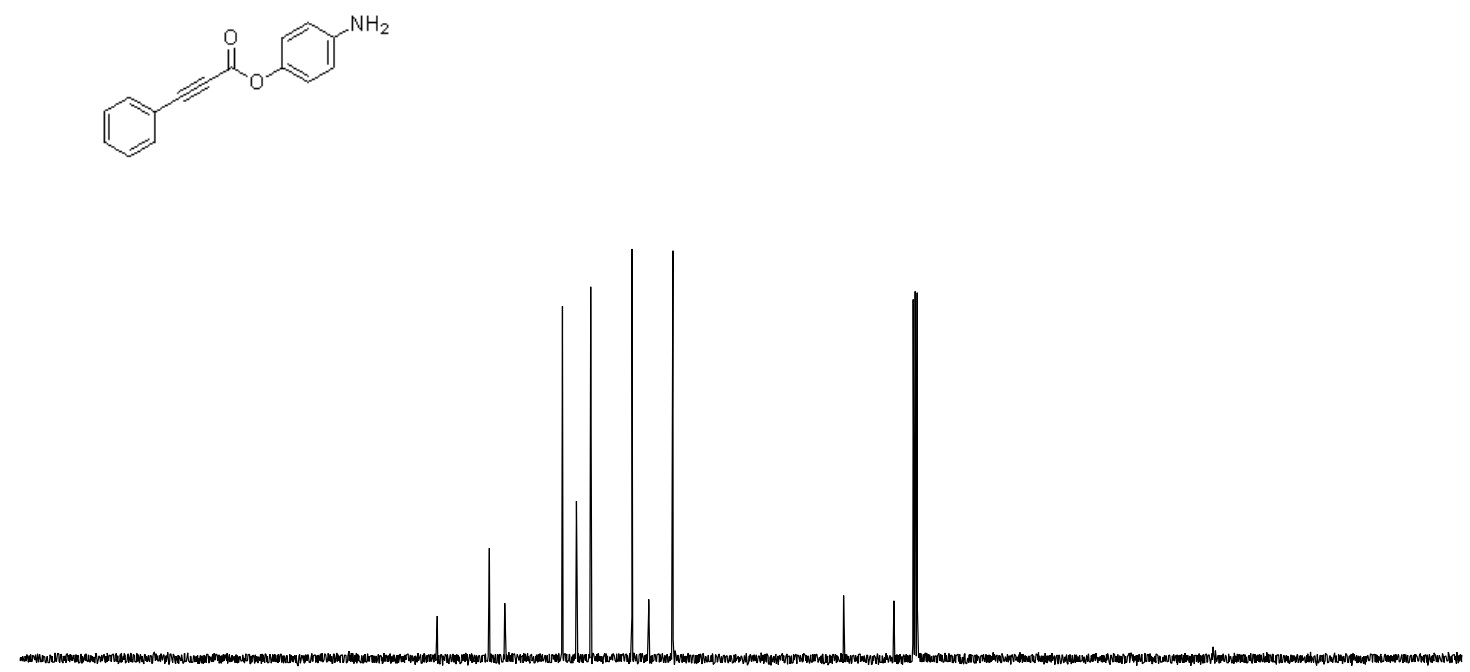

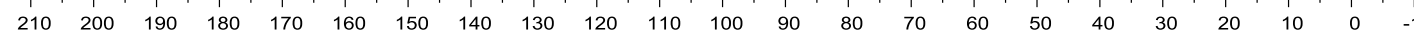


${ }^{1} \mathrm{H}-\mathrm{NMR}$ (400 MHz) spectrum of compound 3ah in $\mathrm{CDCl}_{3}$

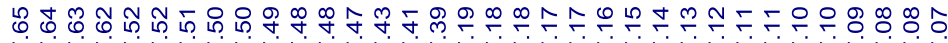

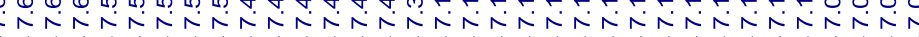
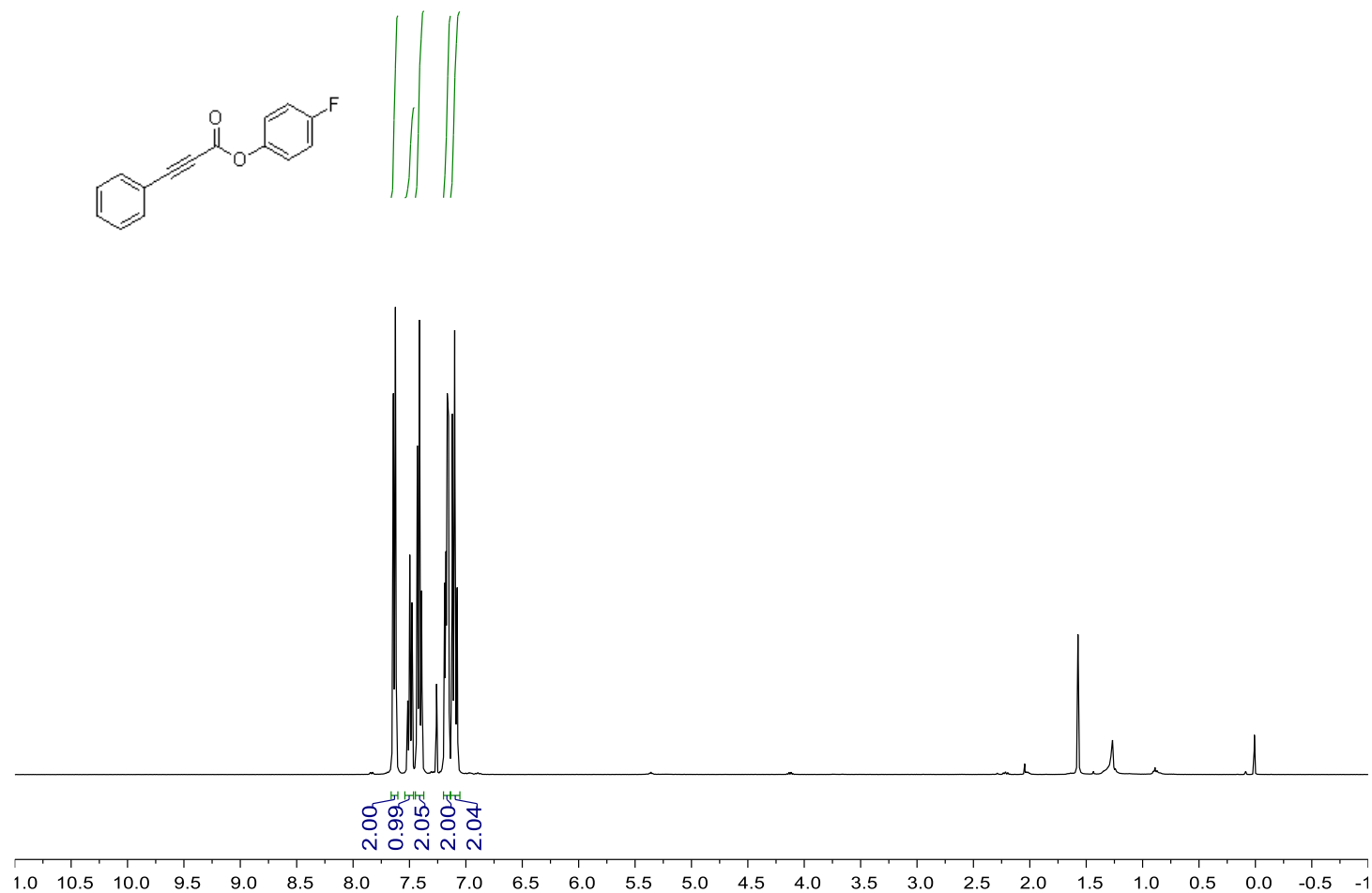

${ }^{13} \mathrm{C}\left\{{ }^{1} \mathrm{H}\right\}$-NMR (100 MHz) spectrum of compound 3ah in $\mathrm{CDCl}_{3}$

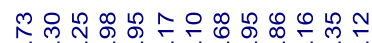

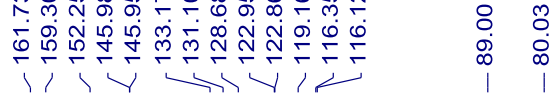

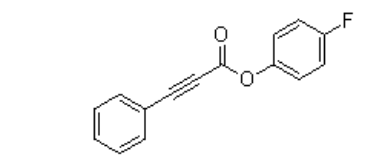

$\begin{array}{llllllllllll}210 & 200 & 190 & 180 & 170 & 160 & 150 & 140 & 130 & 120 & 110 & 100\end{array}$

$90 \quad 80$

60

30

20 
${ }^{1} \mathrm{H}-\mathrm{NMR}\left(400 \mathrm{MHz}\right.$ ) spectrum of compound 3ai in $\mathrm{CDCl}_{3}$

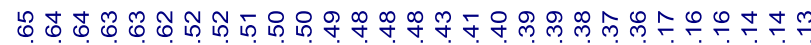

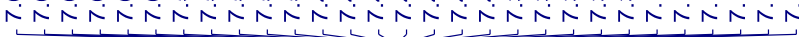
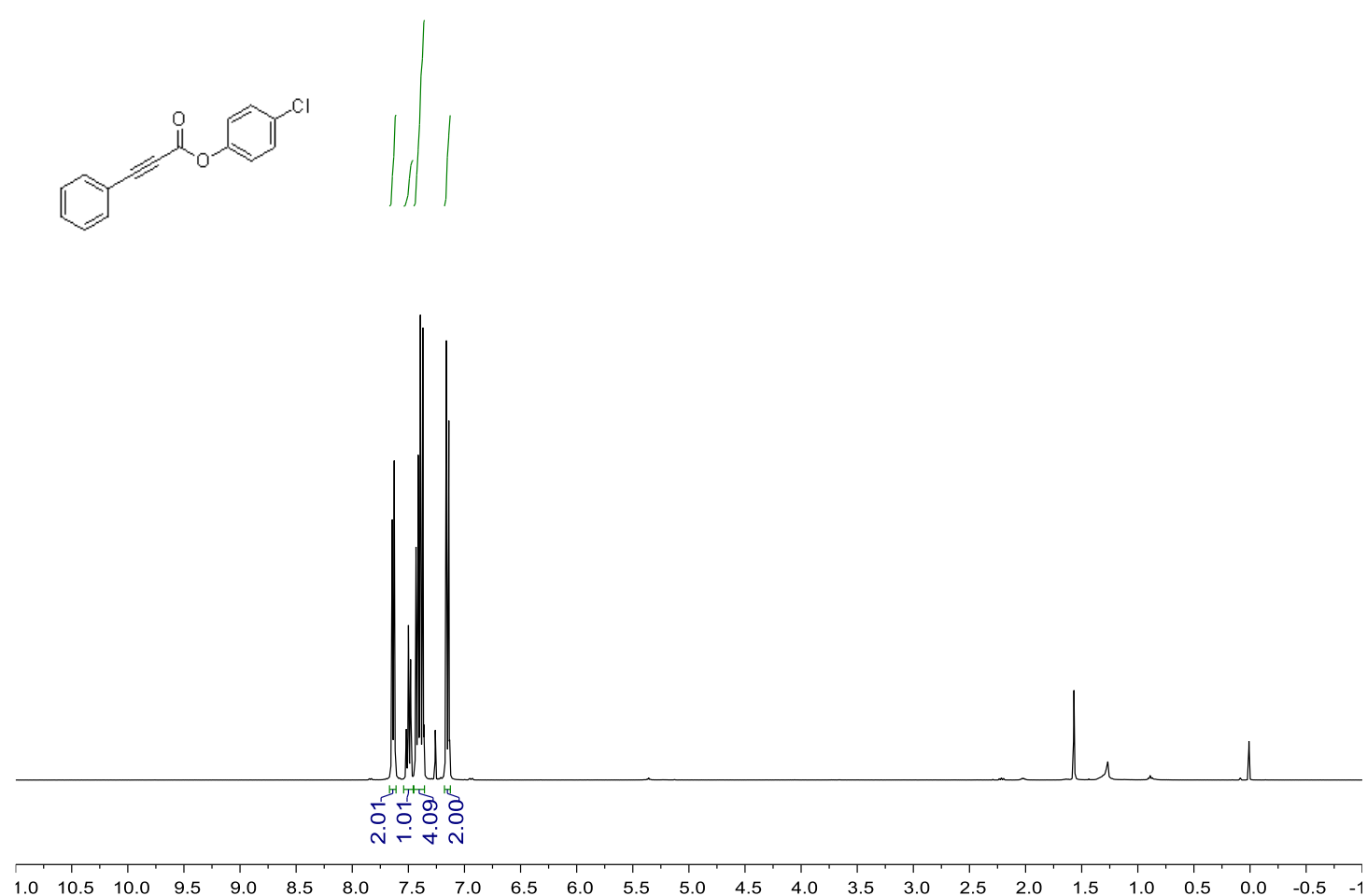

${ }^{13} \mathrm{C}\left\{{ }^{1} \mathrm{H}\right\}$-NMR (100 MHz) spectrum of compound 3ai in $\mathrm{CDCl}_{3}$

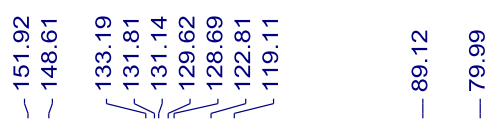
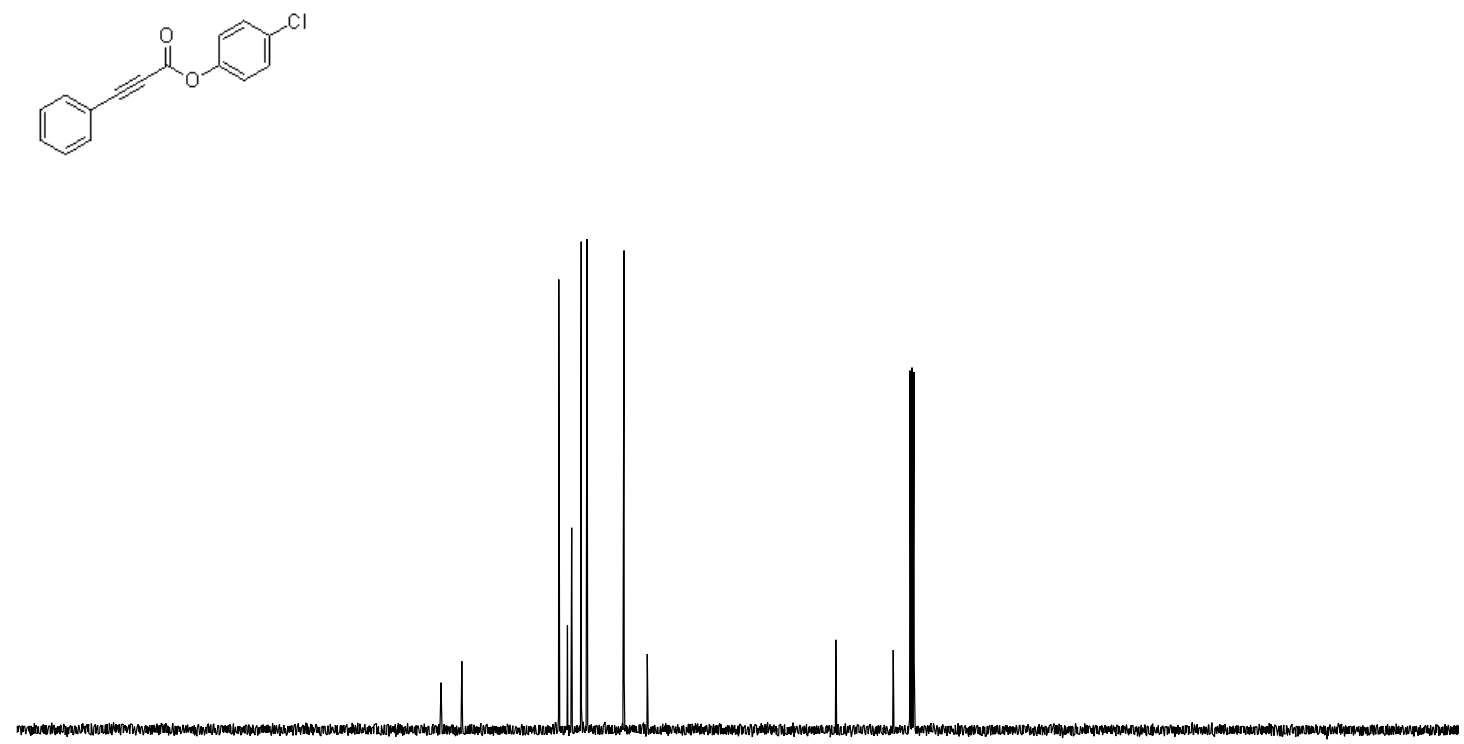

$\begin{array}{llllllllllllllllllllll}210 & 200 & 190 & 180 & 170 & 160 & 150 & 140 & 130 & 120 & 110 & 100 & 90 & 80 & 70 & 60 & 50 & 40 & 30 & 20 & 10 & 0\end{array}$ 
${ }^{1} \mathrm{H}-\mathrm{NMR}(400 \mathrm{MHz})$ spectrum of compound 3aj in $\mathrm{CDCl}_{3}$

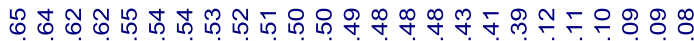

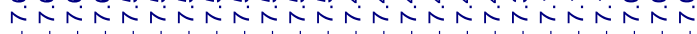
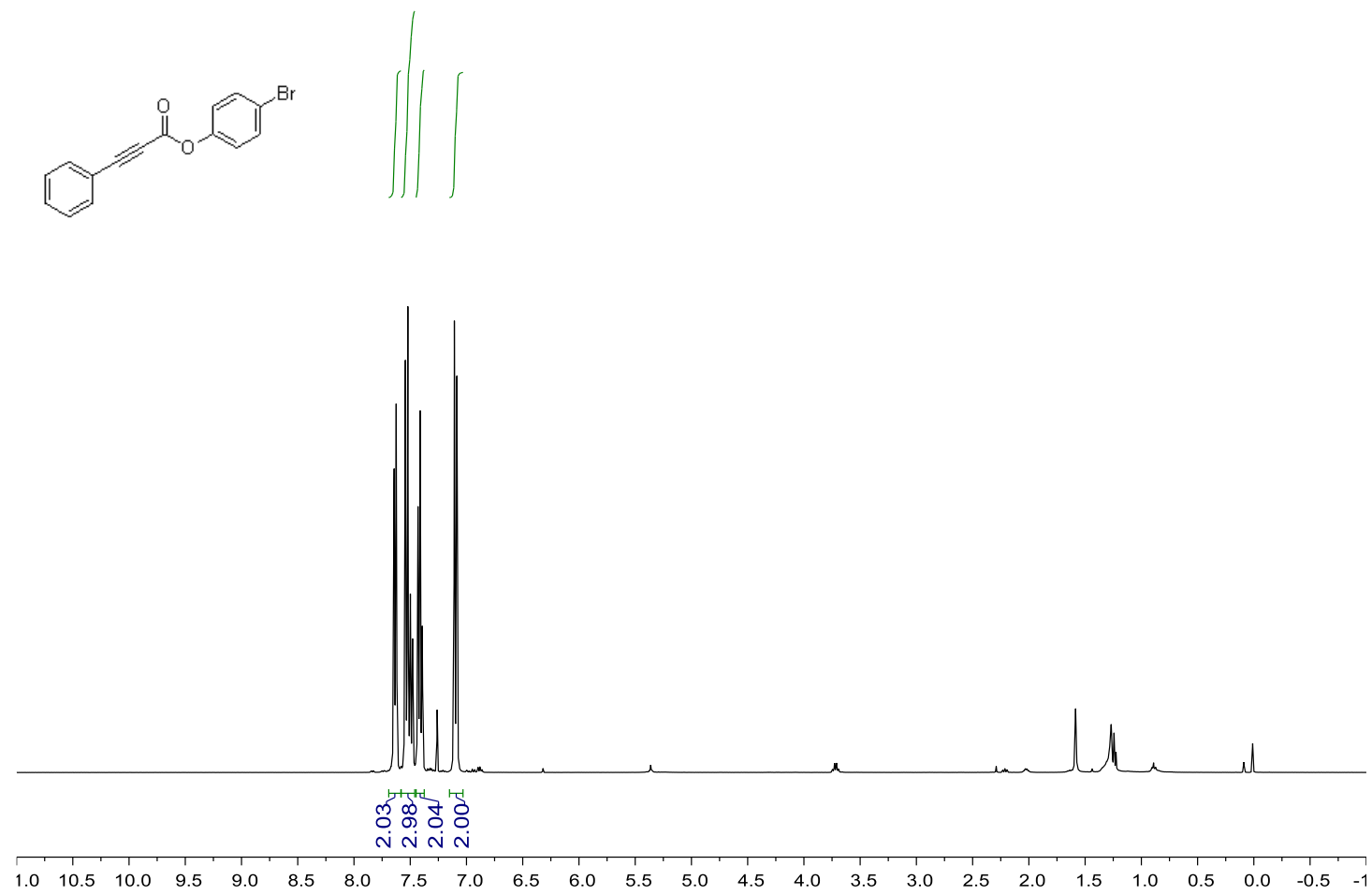

${ }^{13} \mathrm{C}\left\{{ }^{1} \mathrm{H}\right\}$-NMR (100 MHz) spectrum of compound 3aj in $\mathrm{CDCl}_{3}$

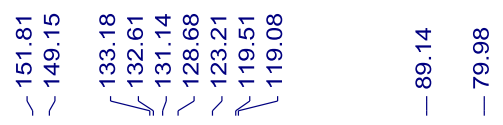

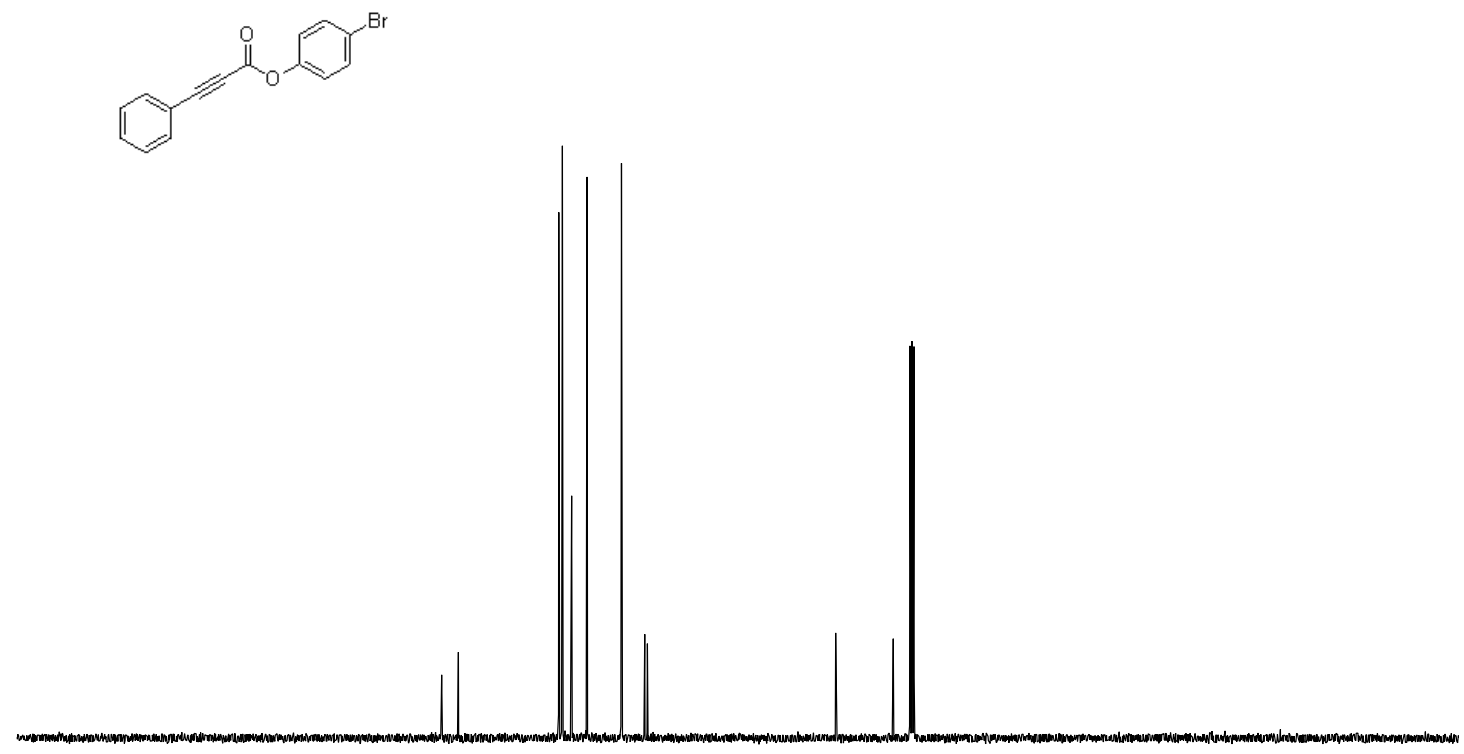

$\begin{array}{llllllllllllllllllllll}210 & 200 & 190 & 180 & 170 & 160 & 150 & 140 & 130 & 120 & 110 & 100 & 90 & 80 & 70 & 60 & 50 & 40 & 30 & 20 & 10 & 0\end{array}$ 
${ }^{1} \mathrm{H}-\mathrm{NMR}\left(400 \mathrm{MHz}\right.$ ) spectrum of compound 3ak in $\mathrm{CDCl}_{3}$

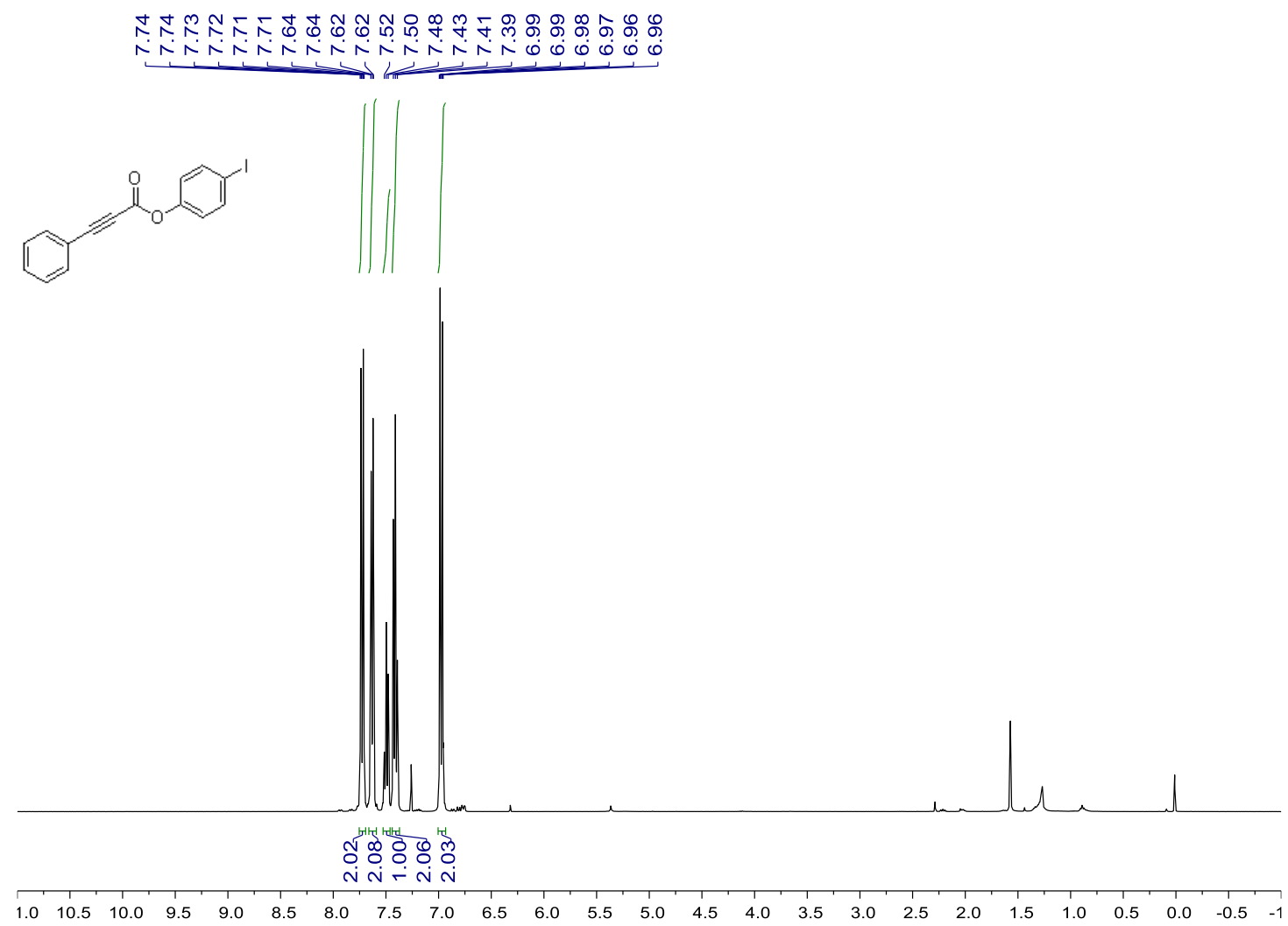

${ }^{13} \mathrm{C}\left\{{ }^{1} \mathrm{H}\right\}$-NMR (100 MHz) spectrum of compound 3ak in $\mathrm{CDCl}_{3}$

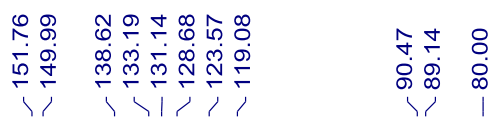

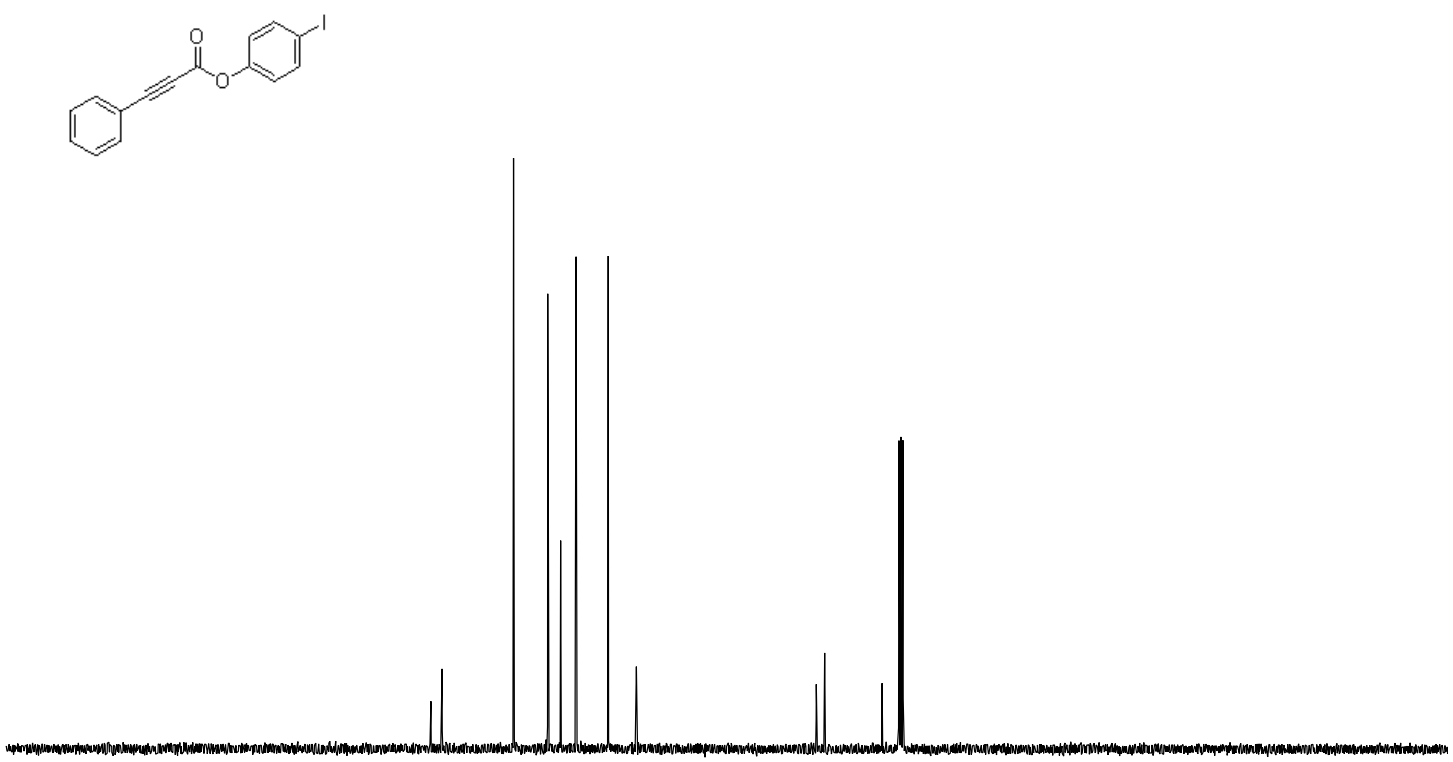

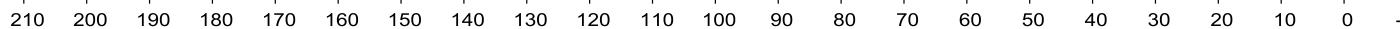


${ }^{1} \mathrm{H}-\mathrm{NMR}\left(400 \mathrm{MHz}\right.$ ) spectrum of compound 3al in $\mathrm{CDCl}_{3}$

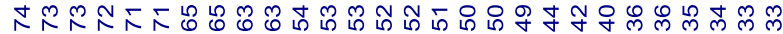

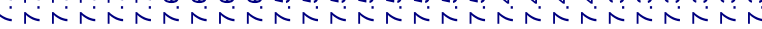
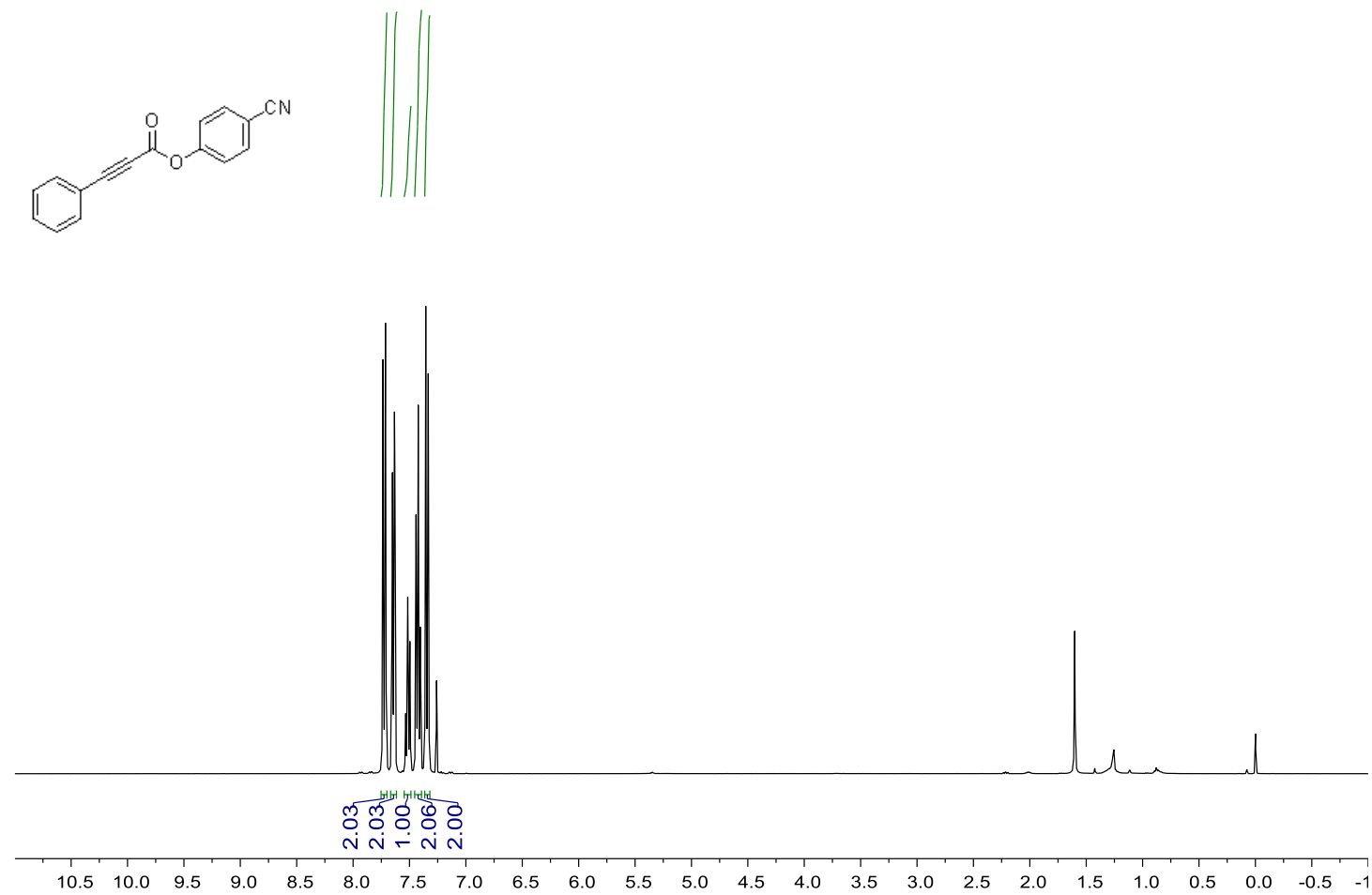

${ }^{13} \mathrm{C}\left\{{ }^{1} \mathrm{H}\right\}$-NMR (100 MHz) spectrum of compound $\mathbf{3 a l}$ in $\mathrm{CDCl}_{3}$

j)
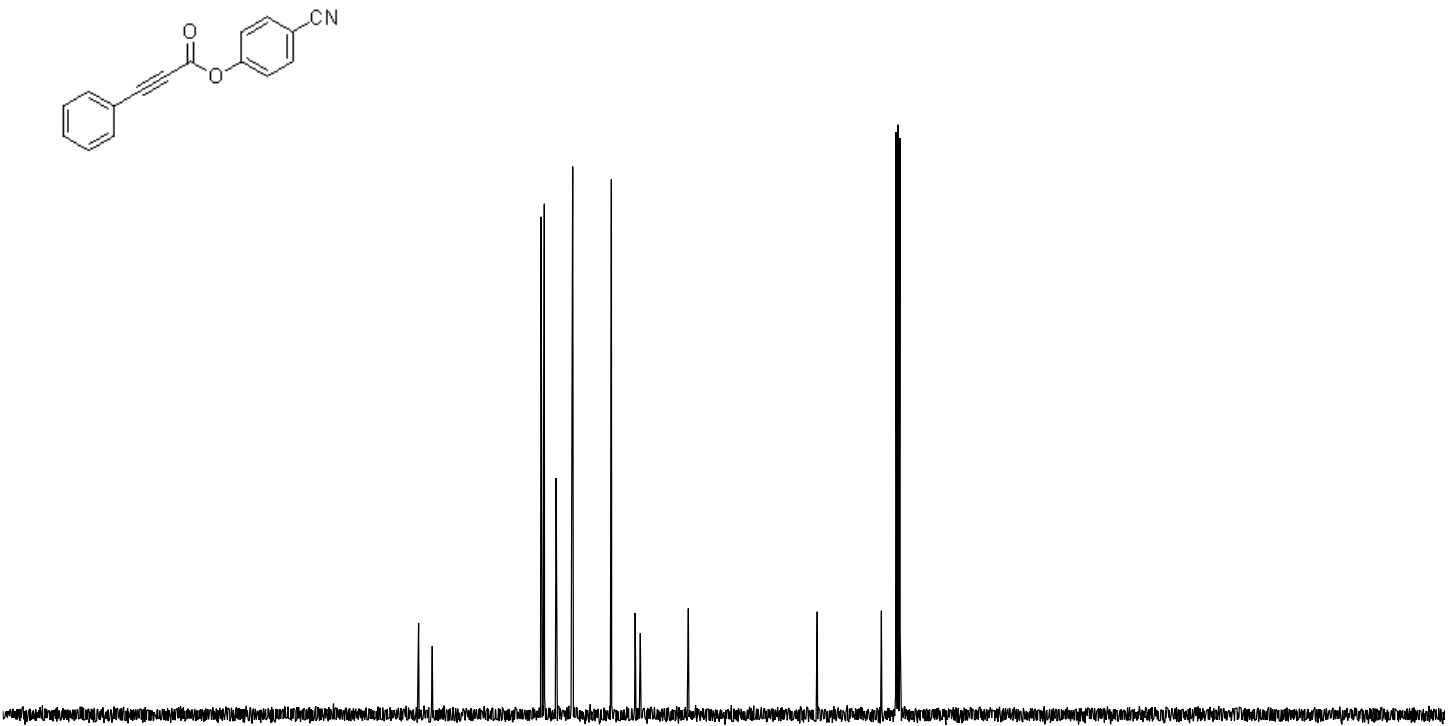

$\begin{array}{lllllllllllllllllllllll}210 & 200 & 190 & 180 & 170 & 160 & 150 & 140 & 130 & 120 & 110 & 100 & 90 & 80 & 70 & 60 & 50 & 40 & 30 & 20 & 10 & 0\end{array}$ 
${ }^{1} \mathrm{H}-\mathrm{NMR}(400 \mathrm{MHz})$ spectrum of compound 3am in $\mathrm{CDCl}_{3}$

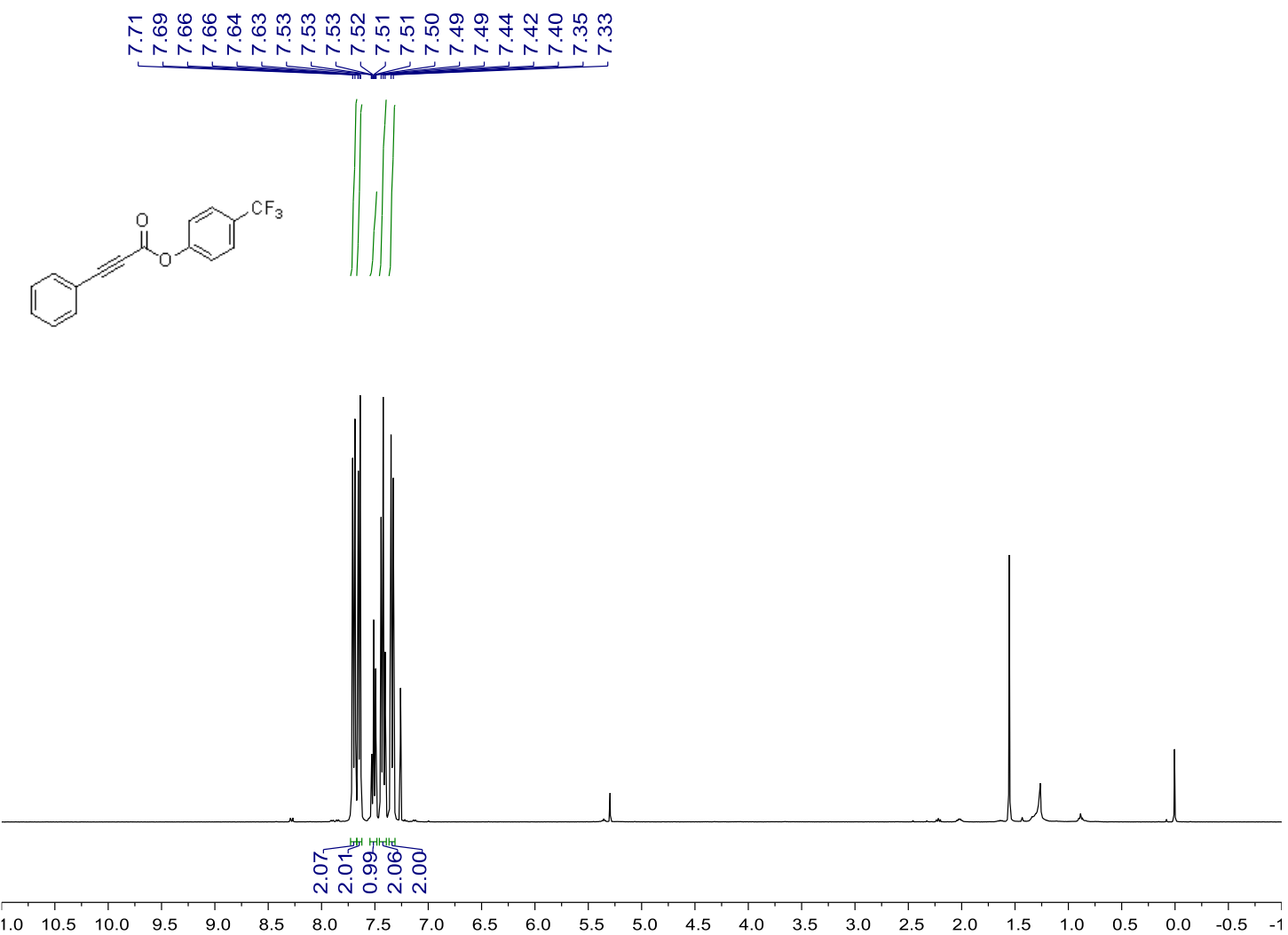

${ }^{13} \mathrm{C}\left\{{ }^{1} \mathrm{H}\right\}$-NMR (100 MHz) spectrum of compound 3am in $\mathrm{CDCl}_{3}$

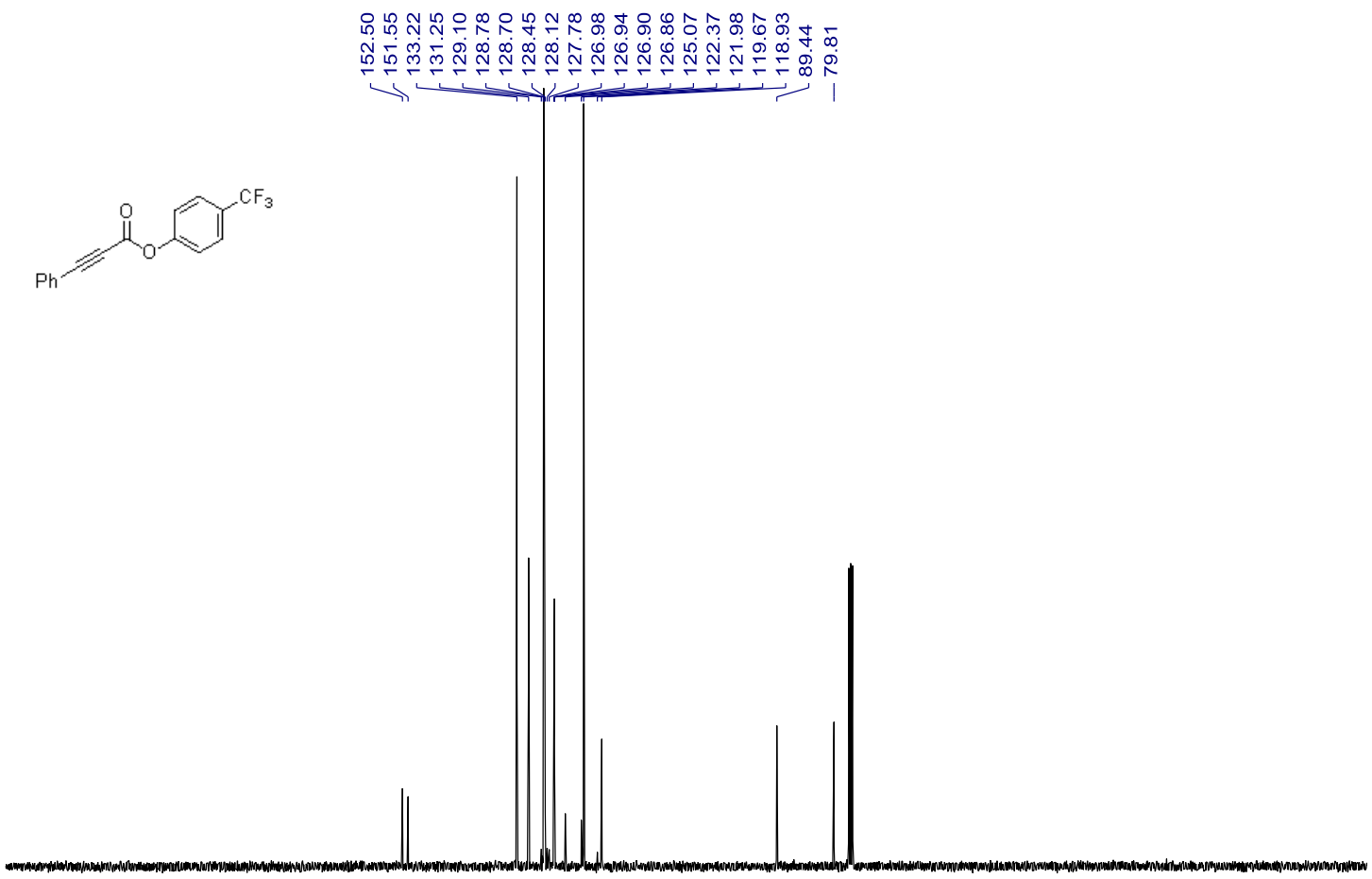

$\begin{array}{lllllllllllllllllllll}210 & 200 & 190 & 180 & 170 & 160 & 150 & 140 & 130 & 120 & 110 & 100 & 90 & 80 & 70 & 60 & 50 & 40 & 30 & 20 & 10\end{array}$ 
${ }^{1} \mathrm{H}-\mathrm{NMR}(400 \mathrm{MHz})$ spectrum of compound 3an in $\mathrm{CDCl}_{3}$

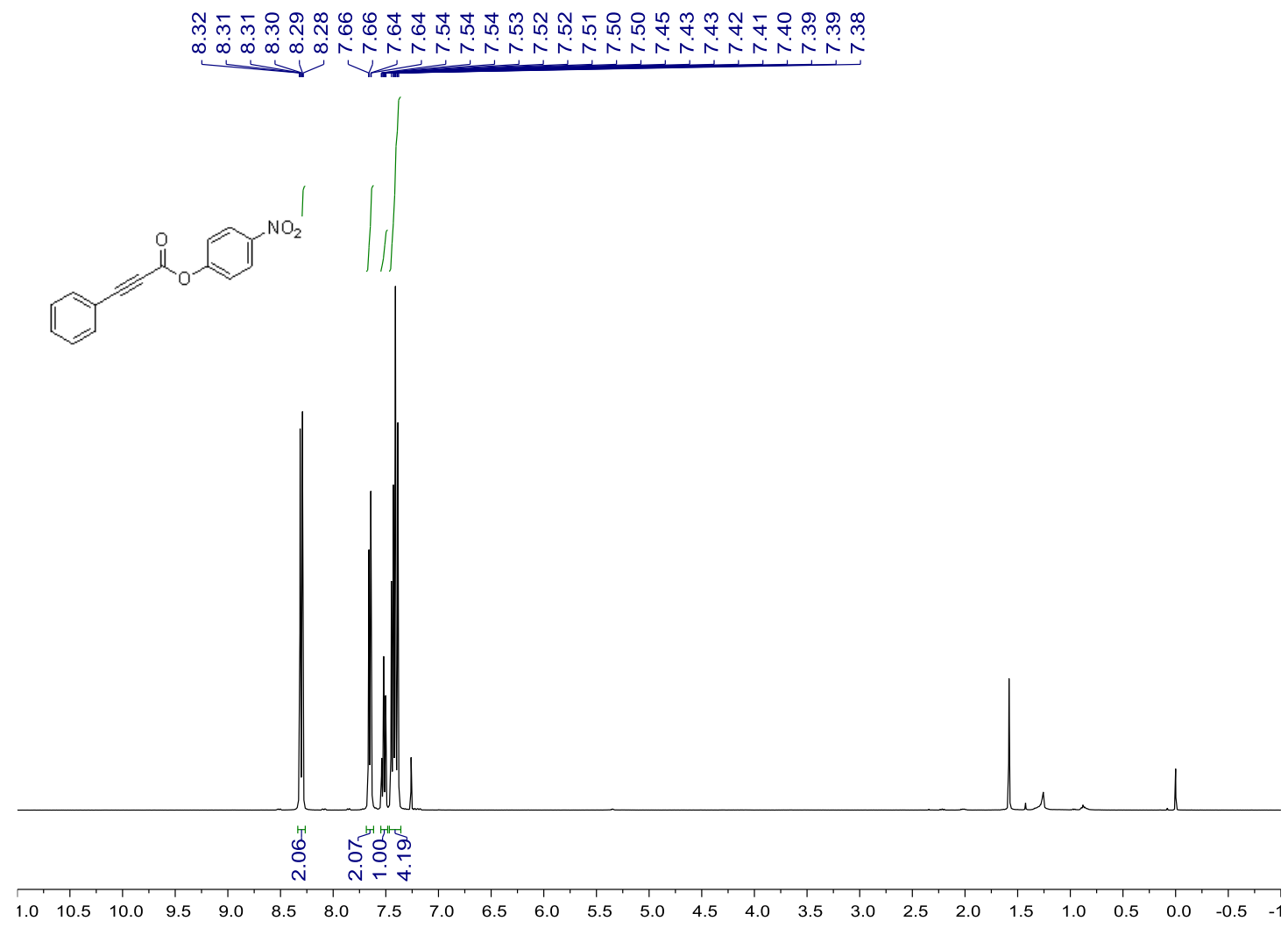

${ }^{13} \mathrm{C}\left\{{ }^{1} \mathrm{H}\right\}$-NMR (100 MHz) spectrum of compound 3an in $\mathrm{CDCl}_{3}$

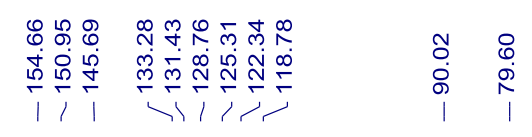

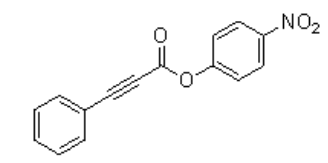

$\begin{array}{llllllllllllllllllllll}210 & 200 & 190 & 180 & 170 & 160 & 150 & 140 & 130 & 120 & 110 & 100 & 90 & 80 & 70 & 60 & 50 & 40 & 30 & 20 & 10 & 0\end{array}$ 
${ }^{1} \mathrm{H}-\mathrm{NMR}\left(400 \mathrm{MHz}\right.$ ) spectrum of compound 3ao in $\mathrm{CDCl}_{3}$

б요요.

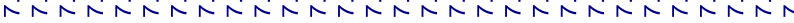

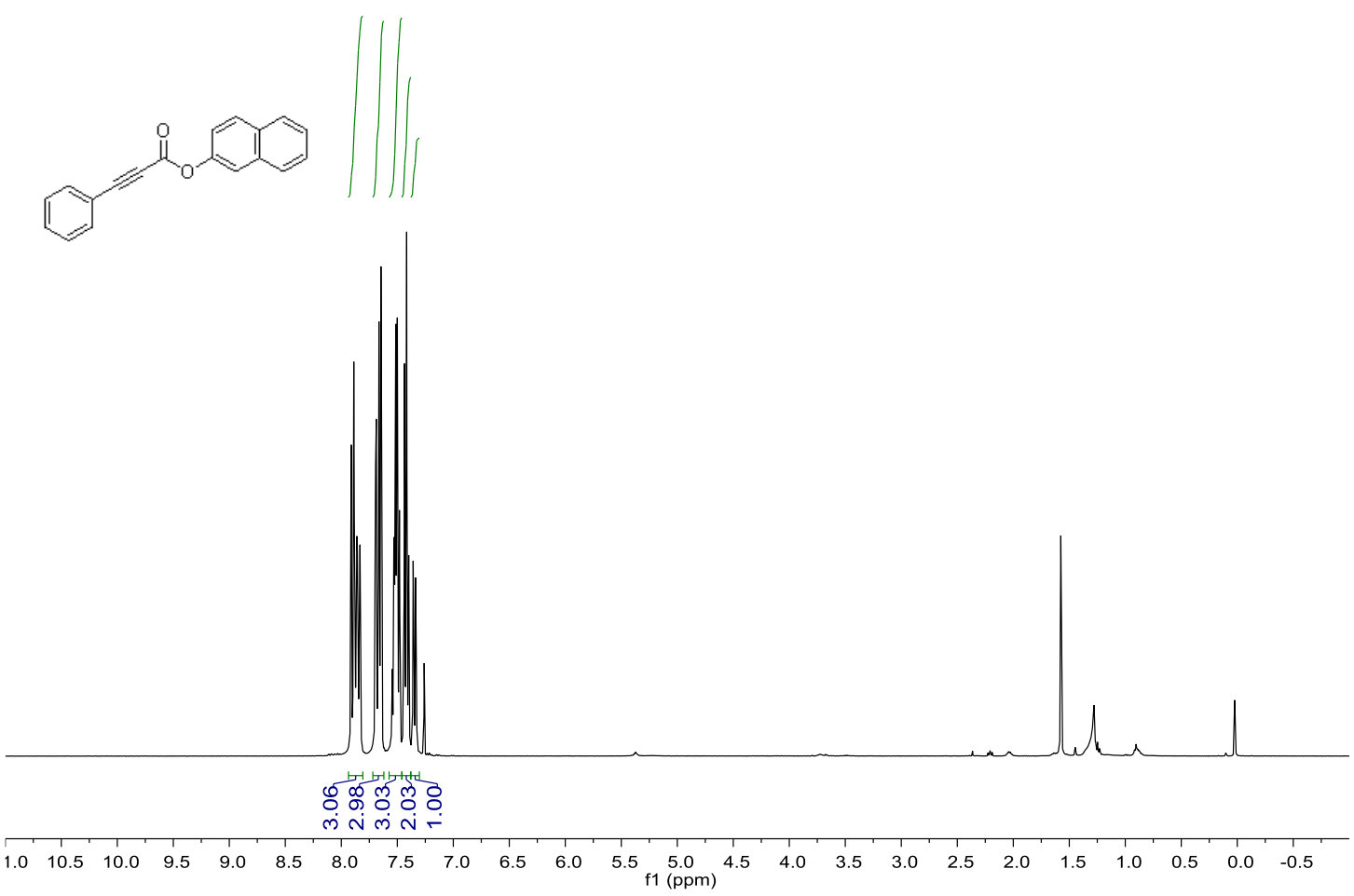

${ }^{13} \mathrm{C}\left\{{ }^{1} \mathrm{H}\right\}$-NMR (100 MHz) spectrum of compound 3ao in $\mathrm{CDCl}_{3}$
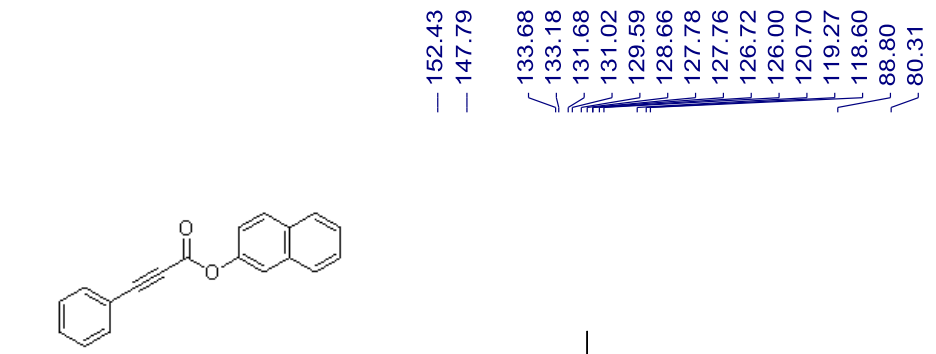

$\begin{array}{lllllllllll}210 & 200 & 190 & 180 & 170 & 160 & 150 & 140 & 130 & 120 & 110 \begin{array}{l}100 \\ \mathrm{f} 1(\mathrm{ppm})\end{array}\end{array}$ 
${ }^{1} \mathrm{H}-\mathrm{NMR}(400 \mathrm{MHz})$ spectrum of compound 3ap in $\mathrm{CDCl}_{3}$

ஜ

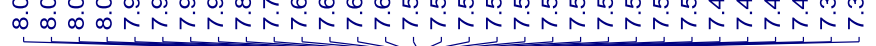

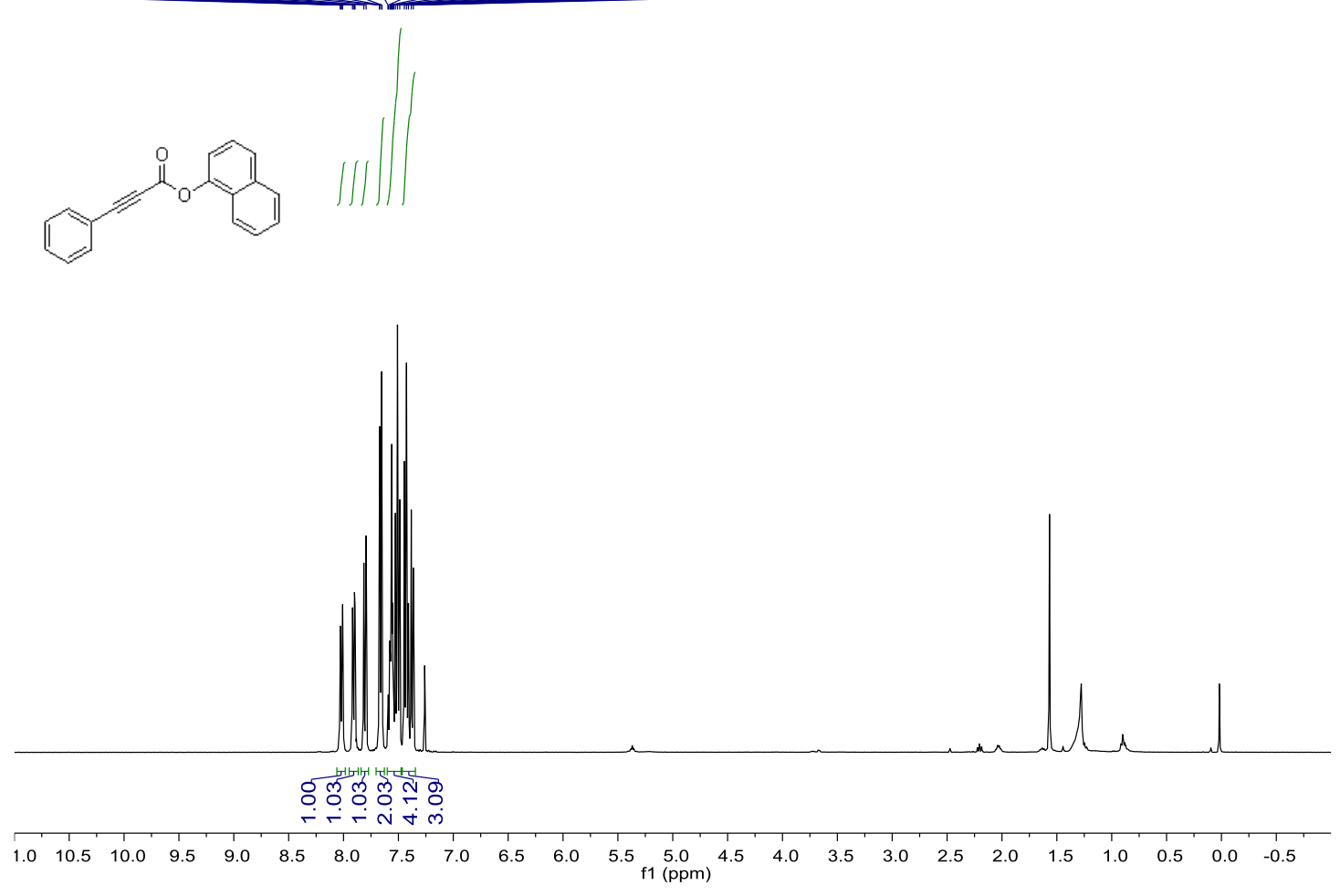

${ }^{13} \mathrm{C}\left\{{ }^{1} \mathrm{H}\right\}-\mathrm{NMR}(100 \mathrm{MHz})$ spectrum of compound 3ap in $\mathrm{CDCl}_{3}$

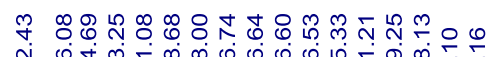

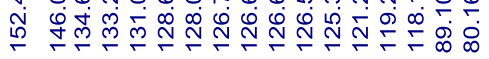

is

। 1 पो।

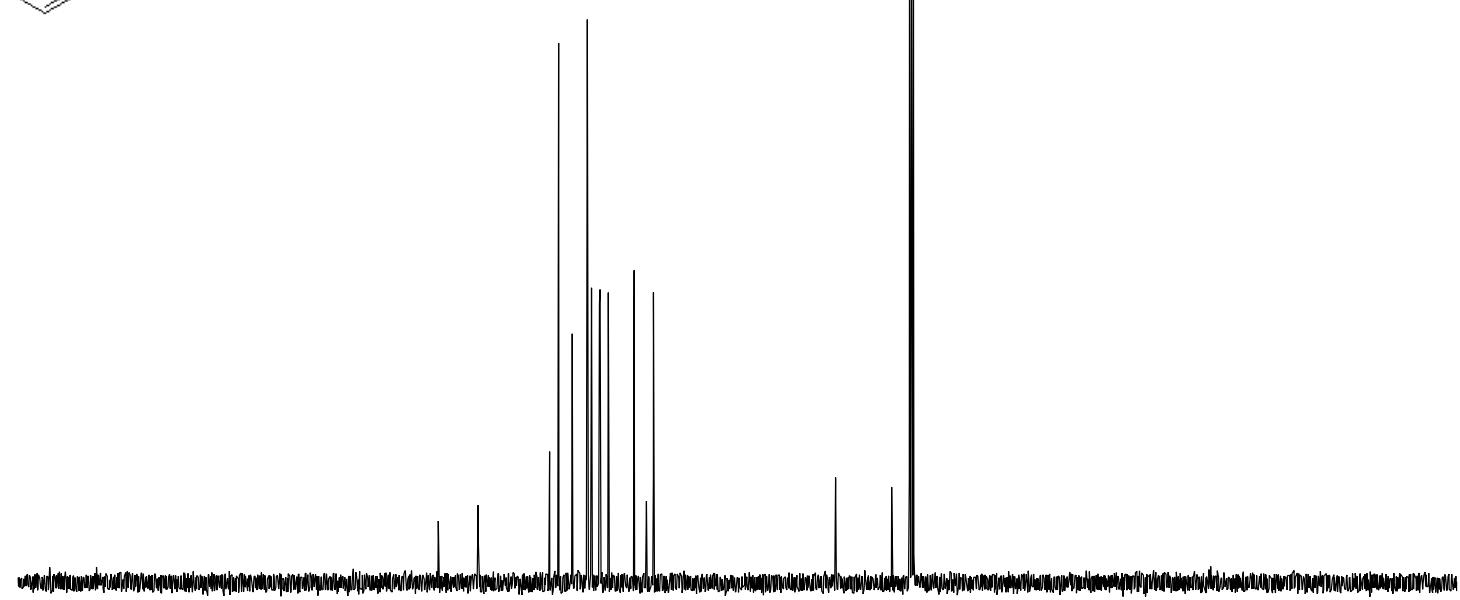

$\begin{array}{llllllllllll}210 & 200 & 190 & 180 & 170 & 160 & 150 & 140 & 130 & 120 & 110 & 100 \\ \mathrm{f} 1(\mathrm{ppm})\end{array}$ 
${ }^{1} \mathrm{H}-\mathrm{NMR}$ (400 MHz) spectrum of compound 3aq in $\mathrm{CDCl}_{3}$

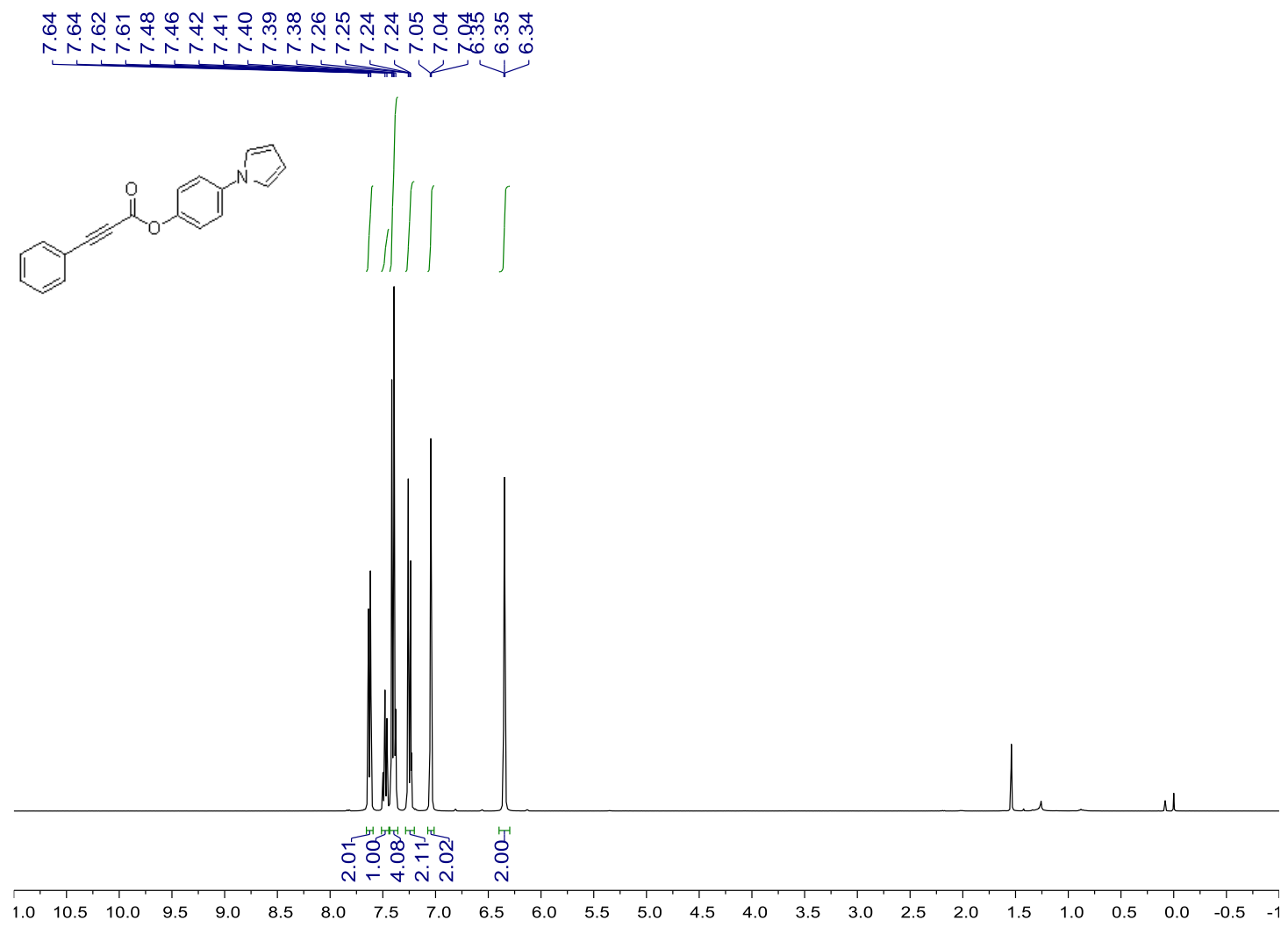

${ }^{13} \mathrm{C}\left\{{ }^{1} \mathrm{H}\right\}$-NMR (100 MHz) spectrum of compound 3aq in $\mathrm{CDCl}_{3}$

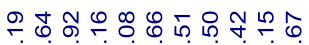

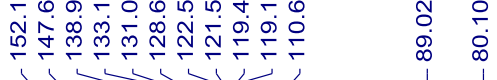
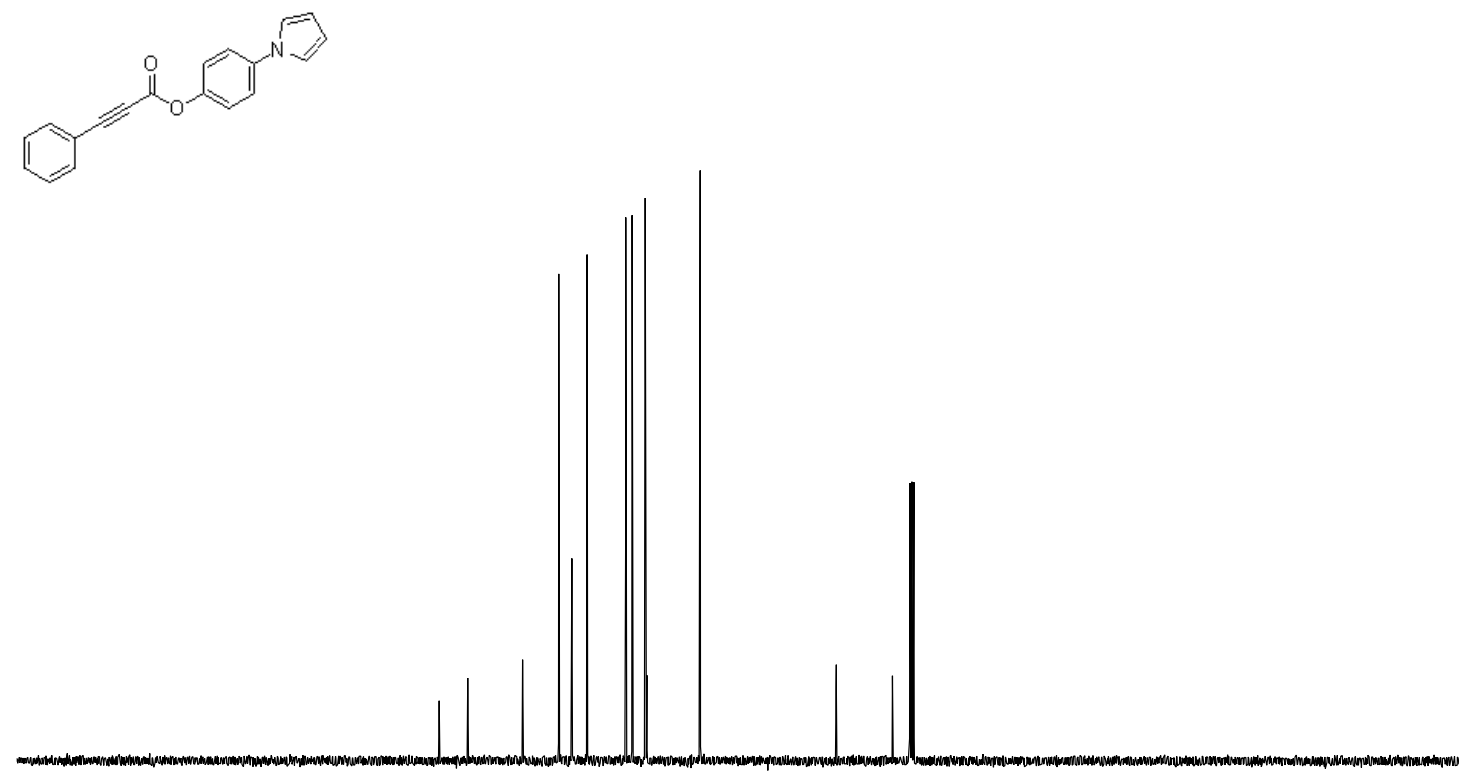

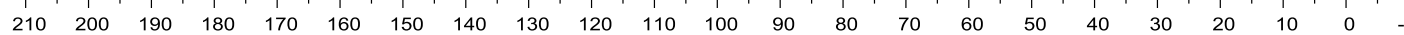


${ }^{1} \mathrm{H}-\mathrm{NMR}\left(400 \mathrm{MHz}\right.$ ) spectrum of compound $\mathbf{3 b a}$ in $\mathrm{CDCl}_{3}$

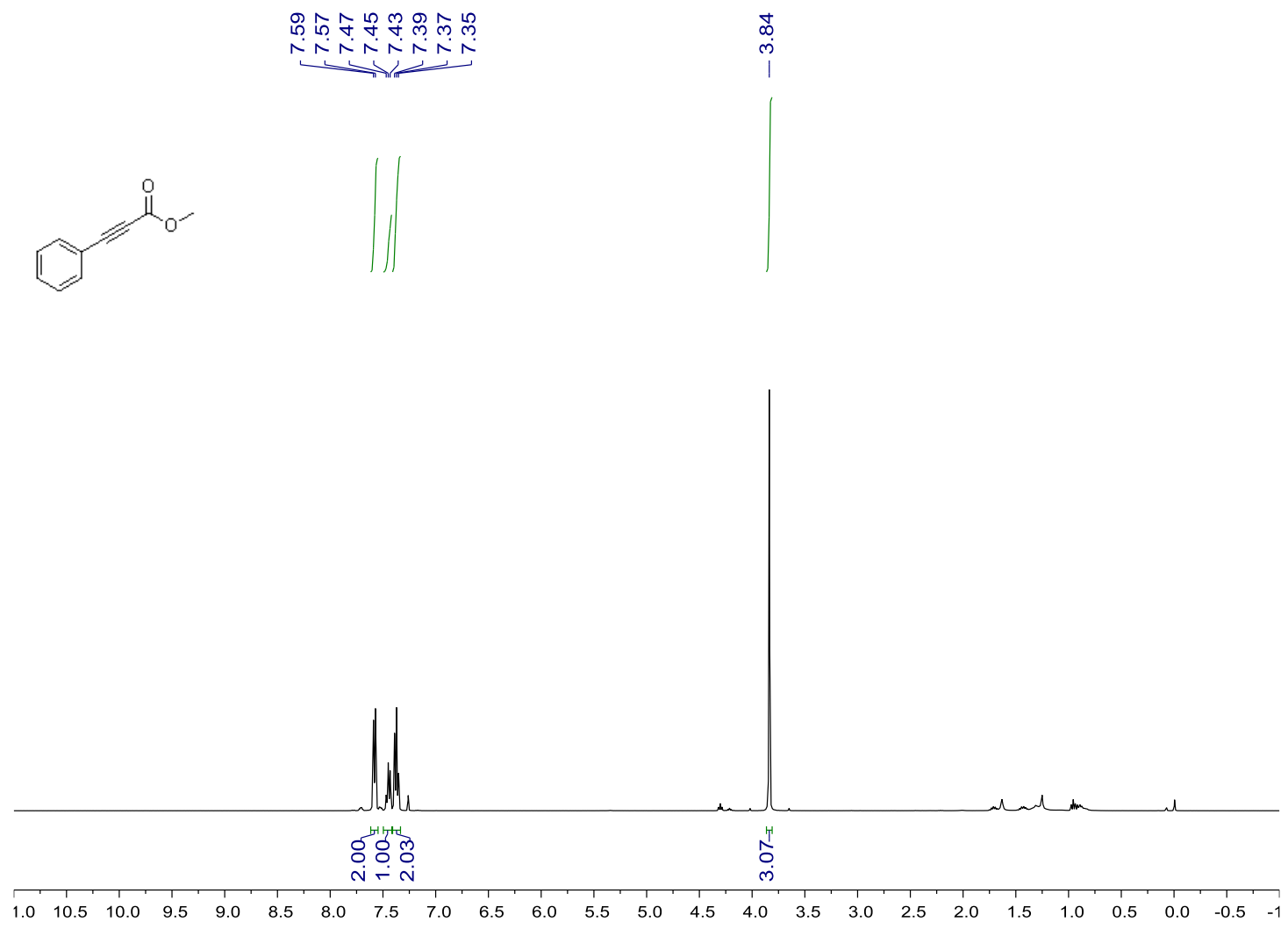

${ }^{13} \mathrm{C}\left\{{ }^{1} \mathrm{H}\right\}$-NMR (100 MHz) spectrum of compound $\mathbf{3 b a}$ in $\mathrm{CDCl}_{3}$

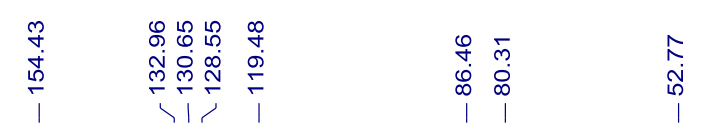
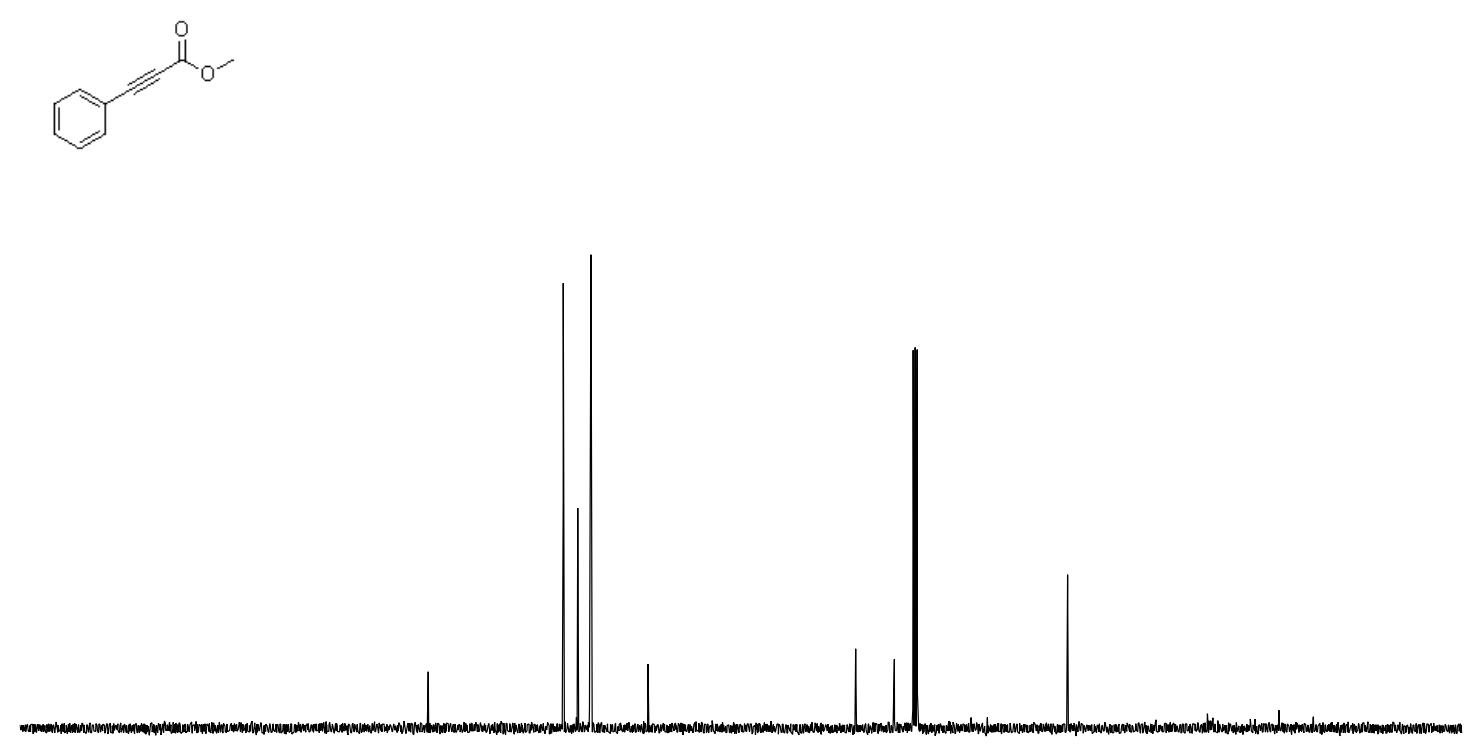

$\begin{array}{llllllllllllllllllllll}210 & 200 & 190 & 180 & 170 & 160 & 150 & 140 & 130 & 120 & 110 & 100 & 90 & 80 & 70 & 60 & 50 & 40 & 30 & 20 & 10 & 0\end{array}$ 
${ }^{1} \mathrm{H}-\mathrm{NMR}(400 \mathrm{MHz})$ spectrum of compound $\mathbf{3 b b}$ in $\mathrm{CDCl}_{3}$
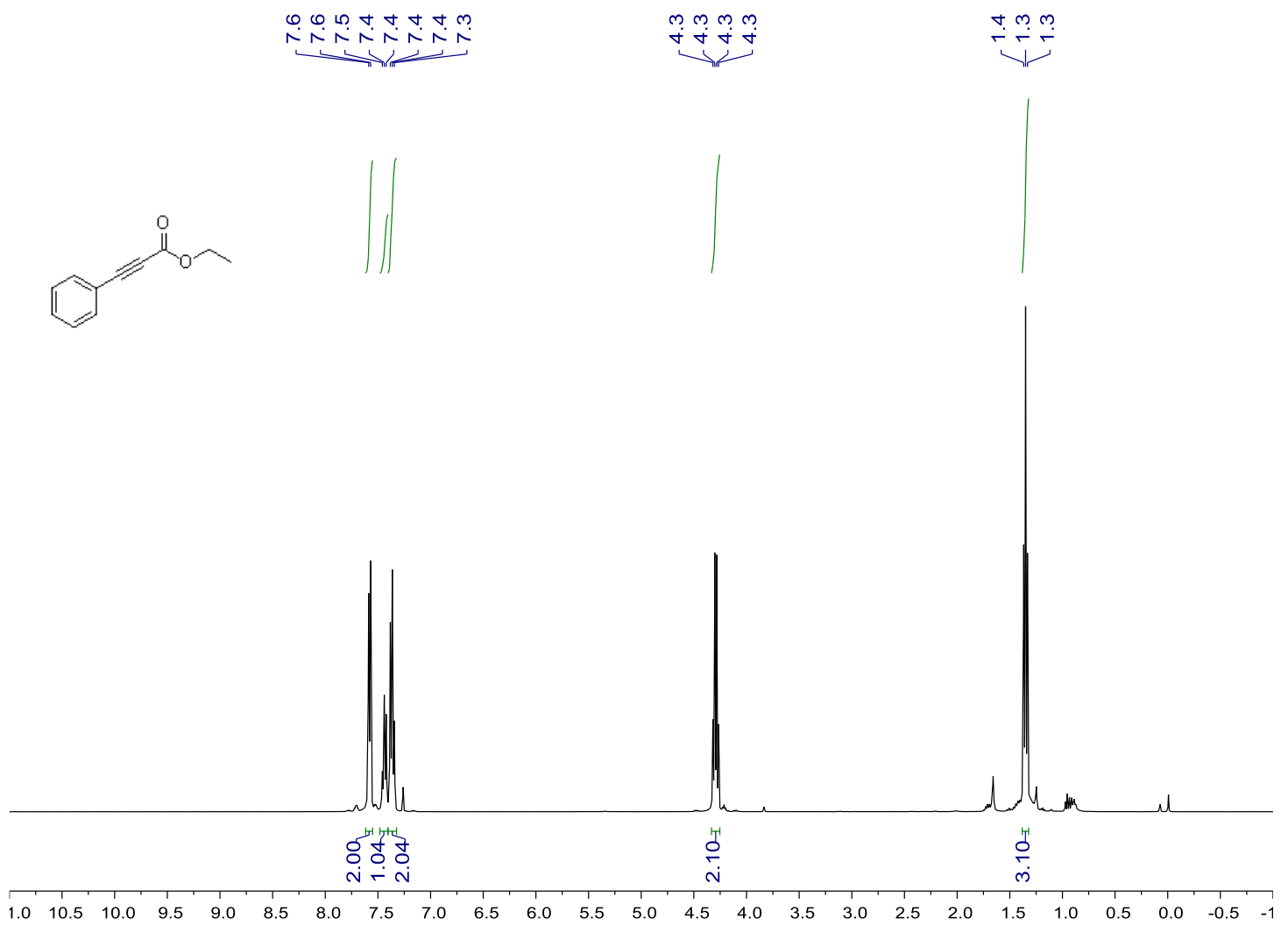

${ }^{13} \mathrm{C}\left\{{ }^{1} \mathrm{H}\right\}$-NMR $(100 \mathrm{MHz})$ spectrum of compound $\mathbf{3 b b}$ in $\mathrm{CDCl}_{3}$
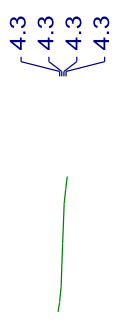

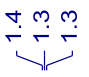

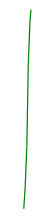

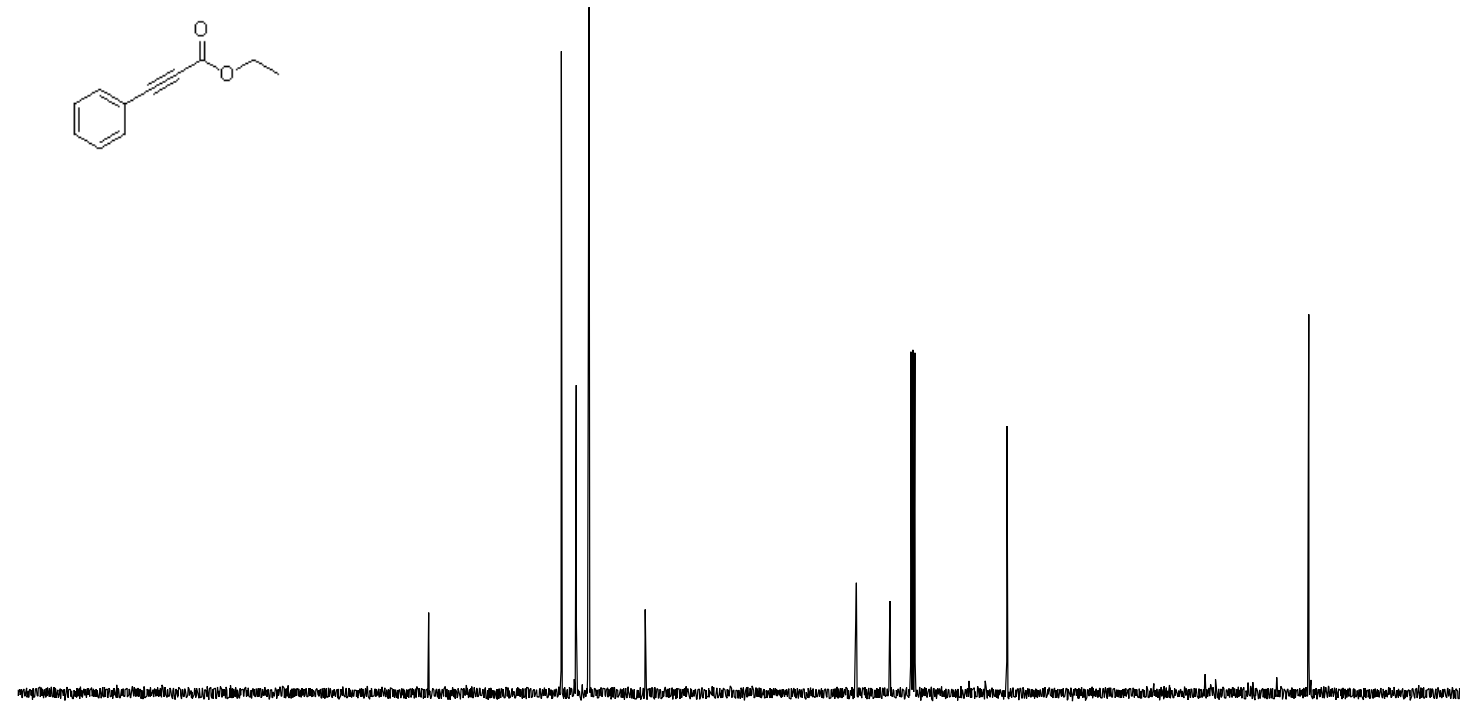

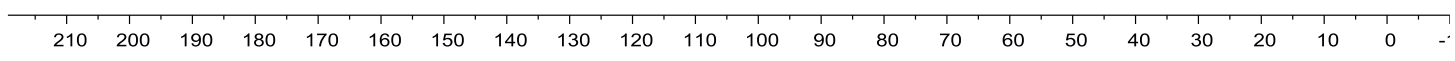

SI-21 
${ }^{1} \mathrm{H}-\mathrm{NMR}(400 \mathrm{MHz})$ spectrum of compound $3 \mathbf{b c}$ in $\mathrm{CDCl}_{3}$

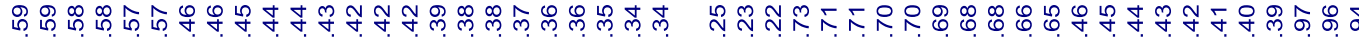

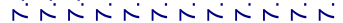
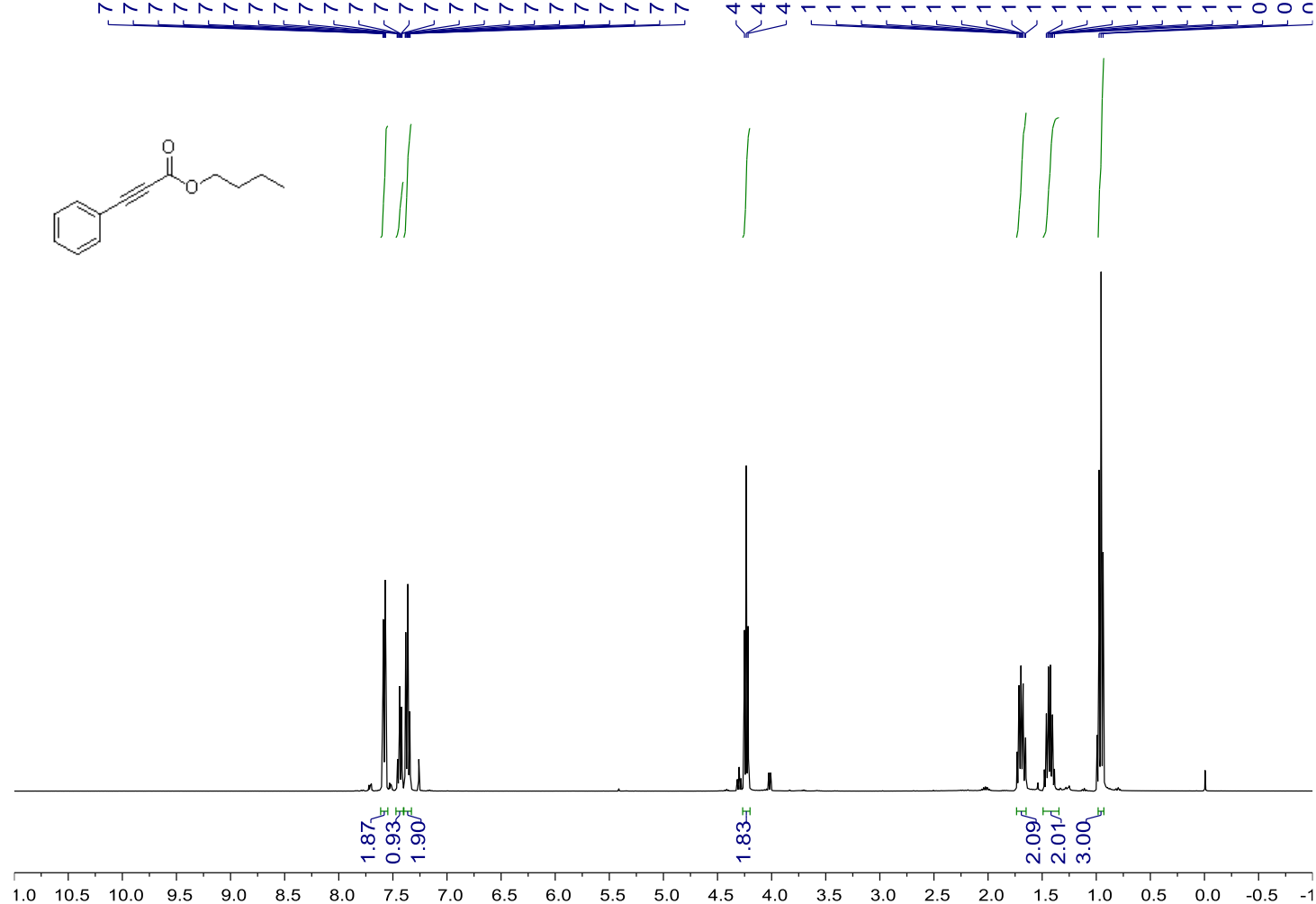

${ }^{13} \mathrm{C}\left\{{ }^{1} \mathrm{H}\right\}$-NMR $(100 \mathrm{MHz})$ spectrum of compound $\mathbf{3 b c}$ in $\mathrm{CDCl}_{3}$

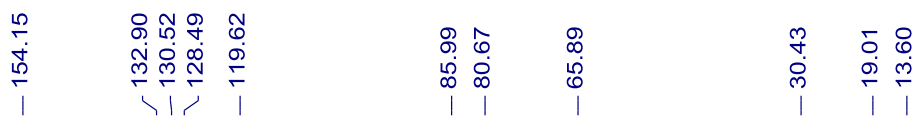
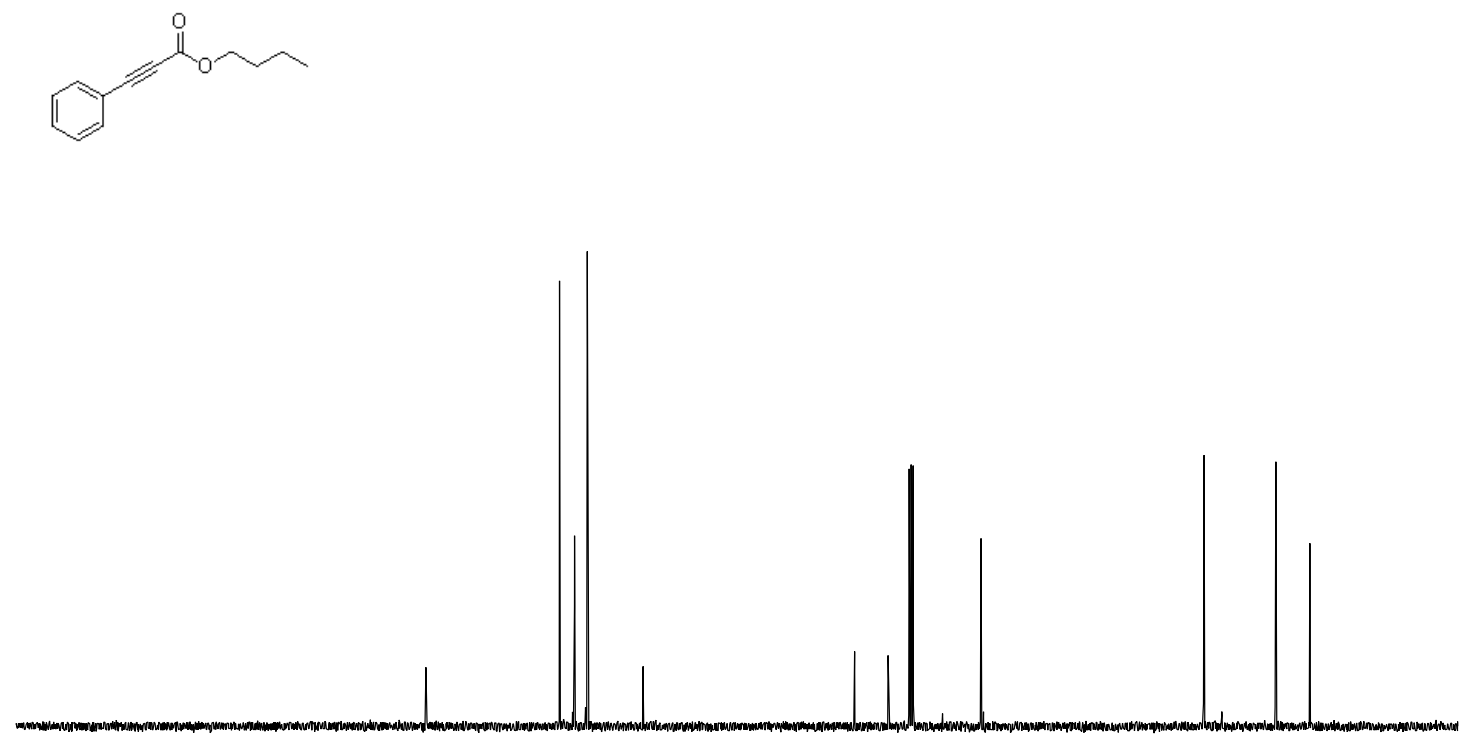

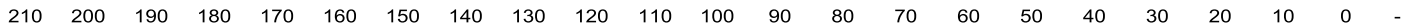


${ }^{1} \mathrm{H}-\mathrm{NMR}\left(400 \mathrm{MHz}\right.$ ) spectrum of compound $\mathbf{3 b d}$ in $\mathrm{CDCl}_{3}$

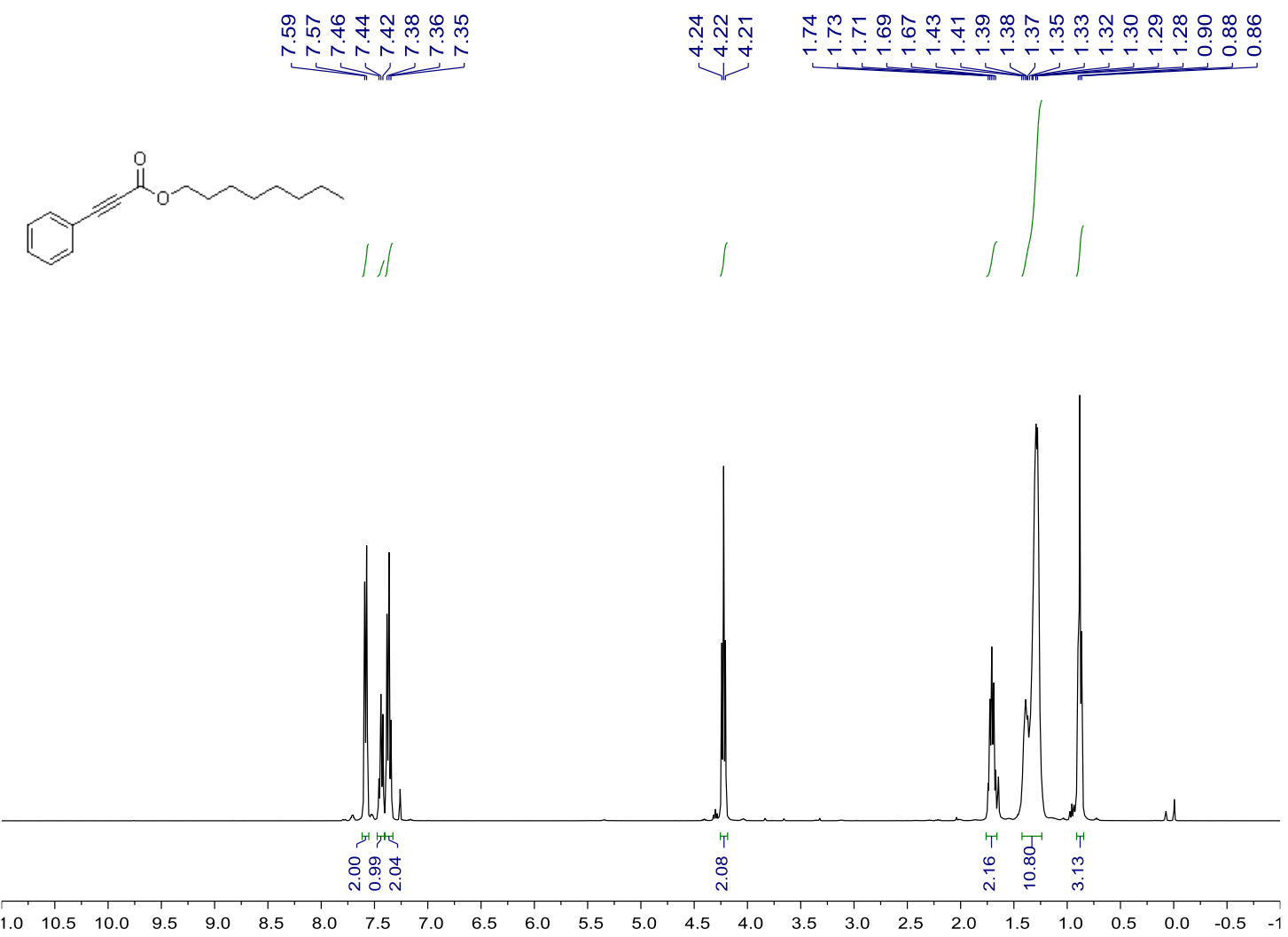

${ }^{13} \mathrm{C}\left\{{ }^{1} \mathrm{H}\right\}$-NMR (100 MHz) spectrum of compound $\mathbf{3 b d}$ in $\mathrm{CDCl}_{3}$

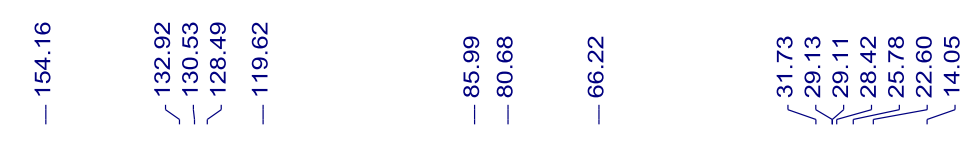
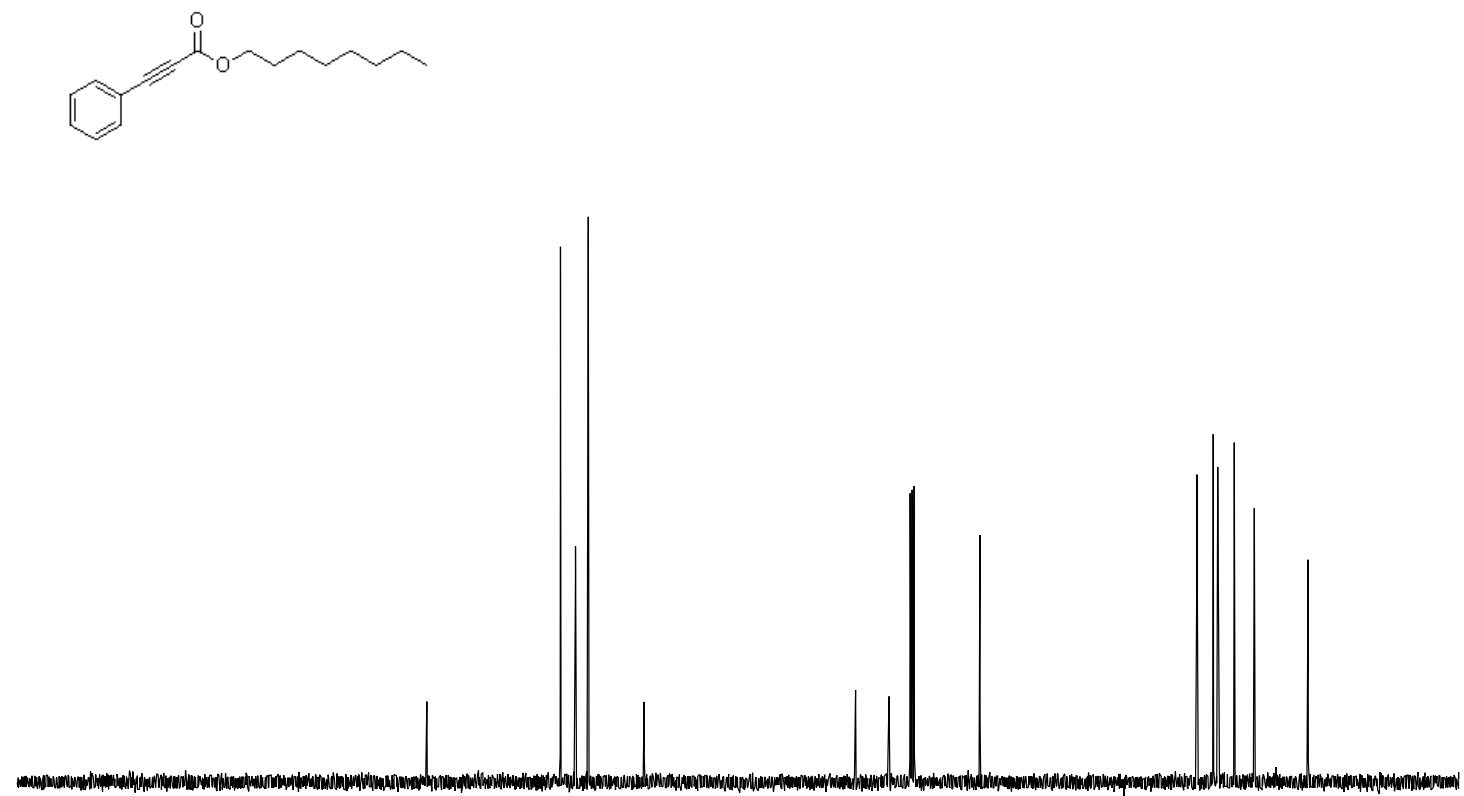

$\begin{array}{llllllllllllllllllllll}210 & 200 & 190 & 180 & 170 & 160 & 150 & 140 & 130 & 120 & 110 & 100 & 90 & 80 & 70 & 60 & 50 & 40 & 30 & 20 & 10 & 0\end{array}$ 
${ }^{1} \mathrm{H}-\mathrm{NMR}$ (400 MHz) spectrum of compound 3be in $\mathrm{CDCl}_{3}$
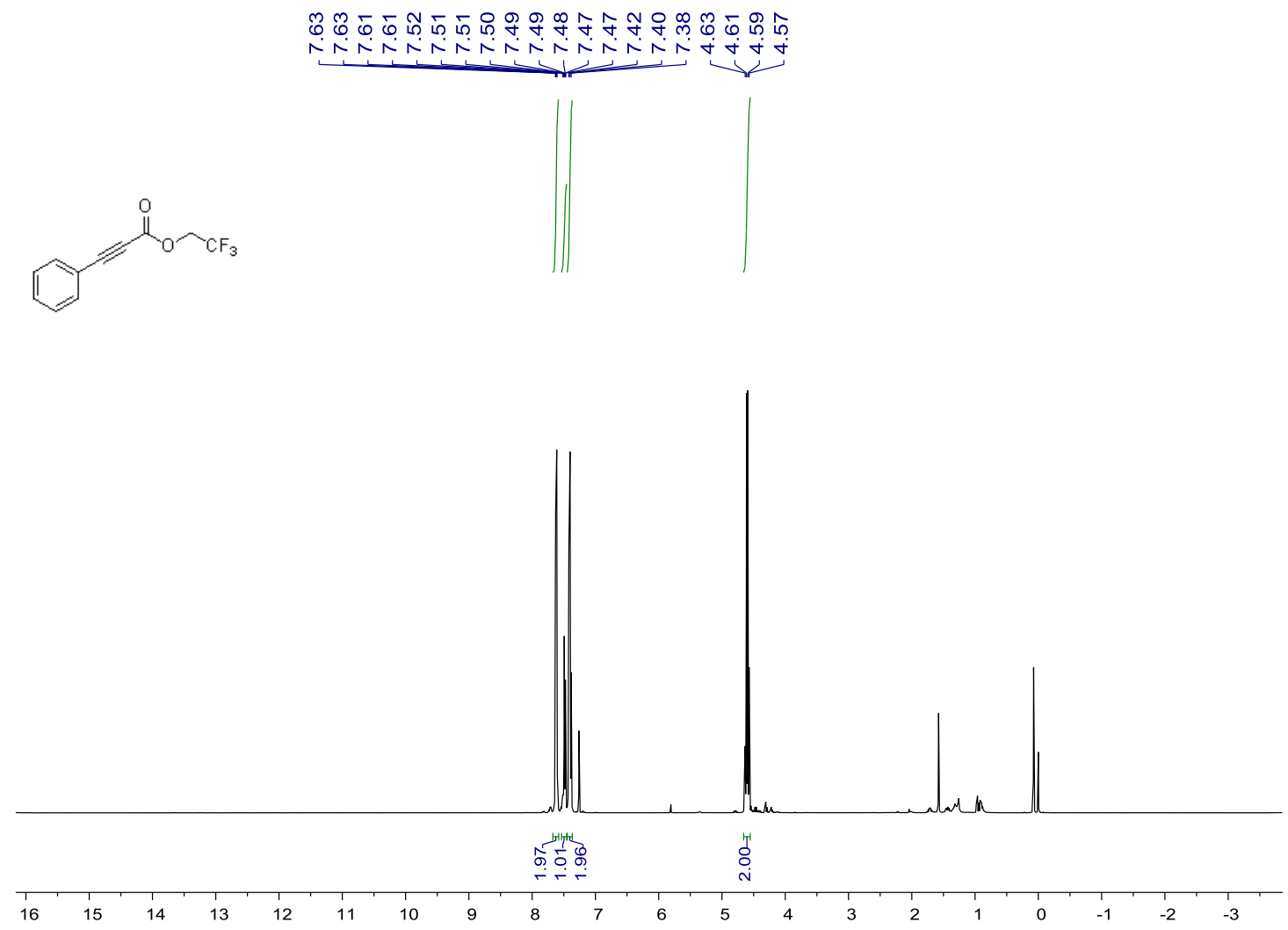

${ }^{13} \mathrm{C}\left\{{ }^{1} \mathrm{H}\right\}$-NMR (100 MHz) spectrum of compound 3 be in $\mathrm{CDCl}_{3}$

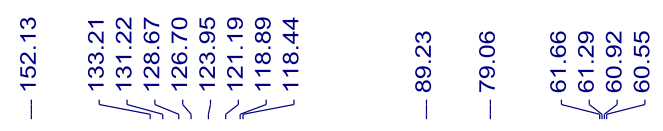
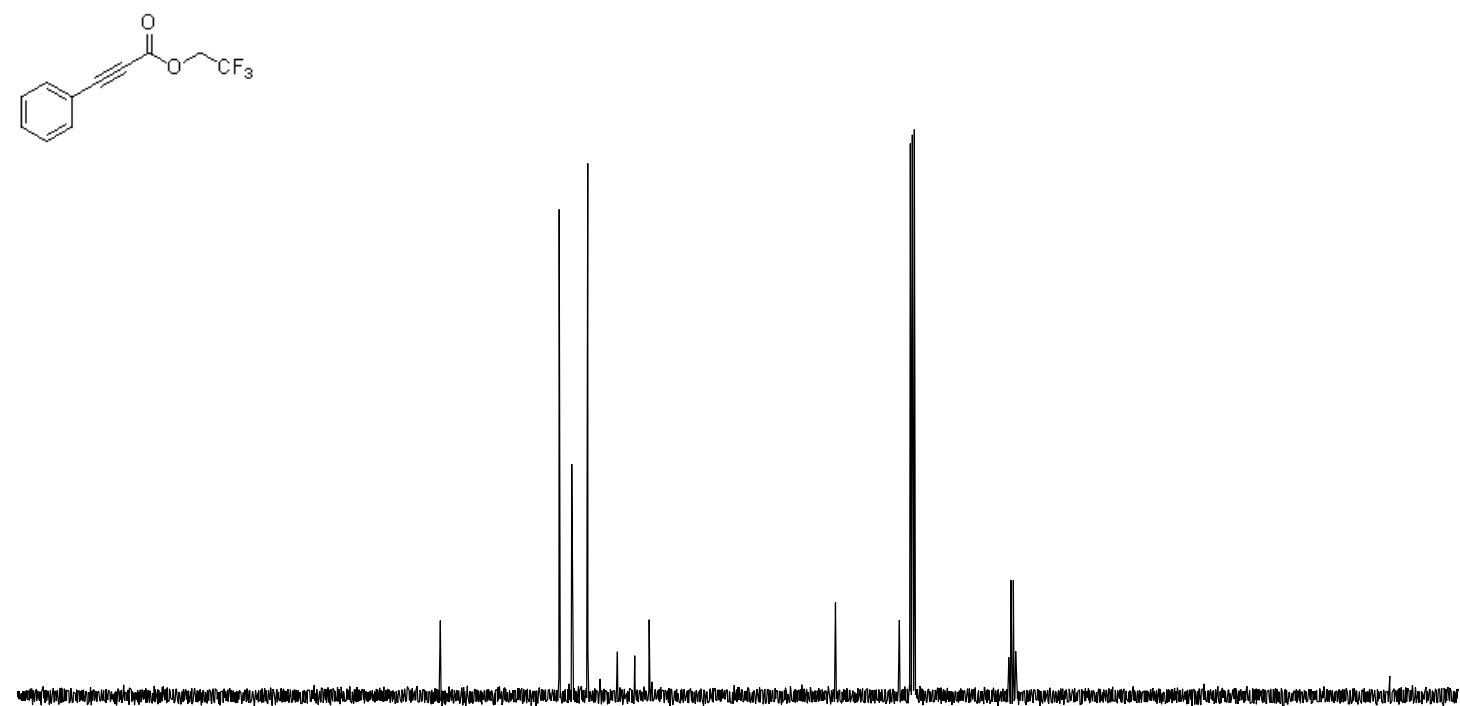

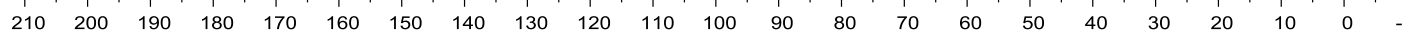


${ }^{1} \mathrm{H}-\mathrm{NMR}(400 \mathrm{MHz})$ spectrum of compound $3 \mathbf{b f}$ in $\mathrm{CDCl}_{3}$

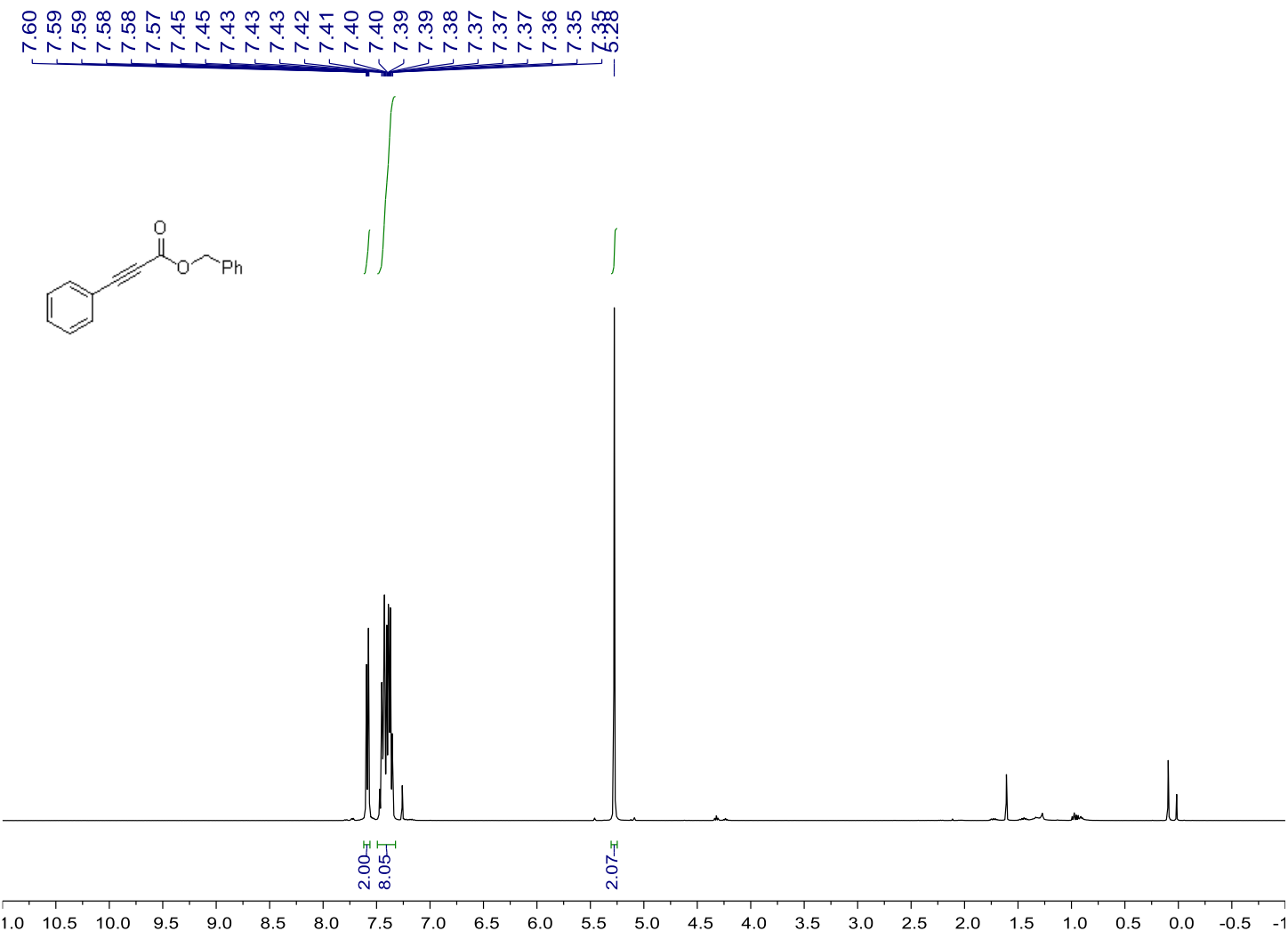

${ }^{13} \mathrm{C}\left\{{ }^{1} \mathrm{H}\right\}$-NMR (100 MHz) spectrum of compound $3 \mathbf{b f}$ in $\mathrm{CDCl}_{3}$
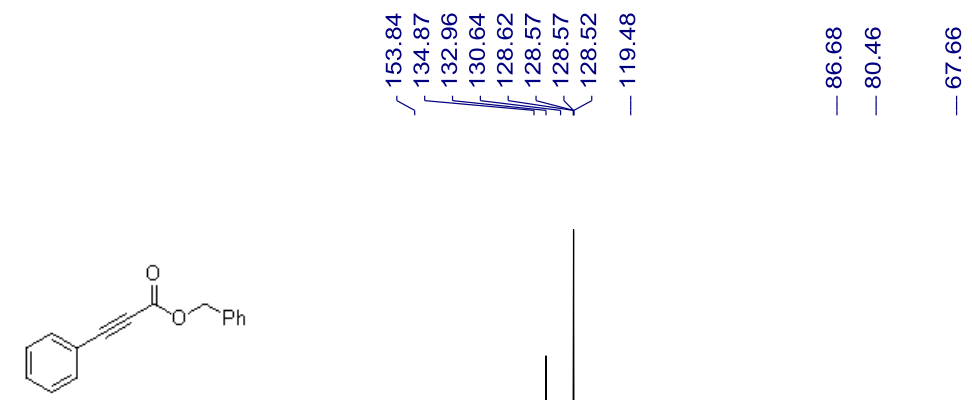

$\begin{array}{ccc}0 & 0 & 0 \\ 0 & 0 & 0 \\ 1 & 1 & 1\end{array}$

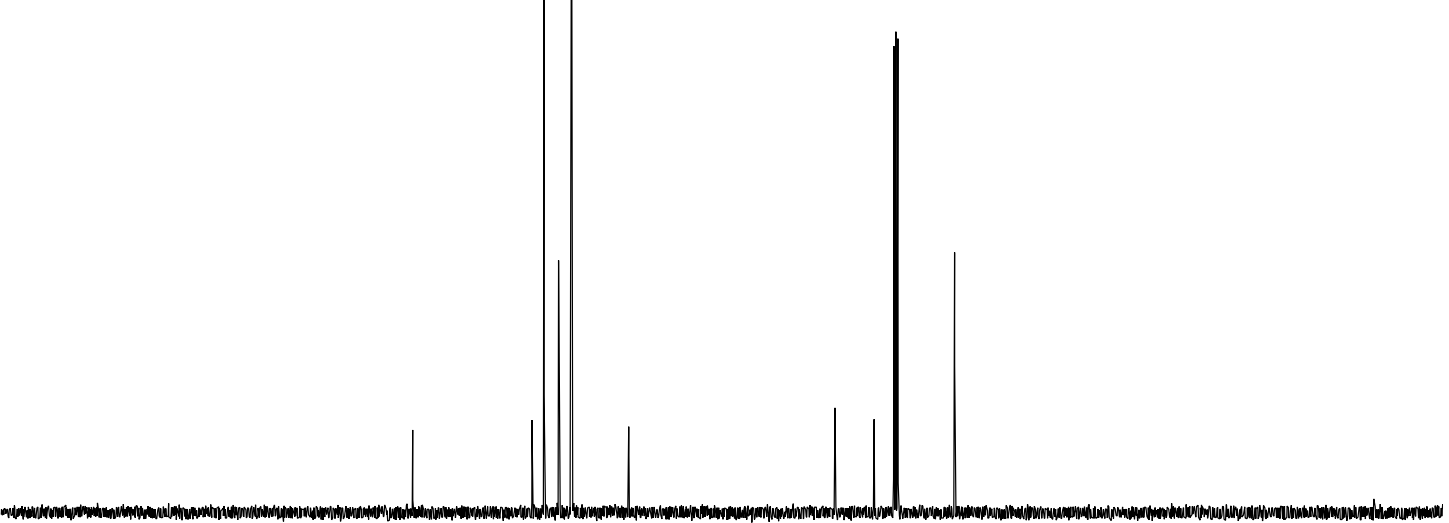

$\begin{array}{llllllllllllllllllllll}210 & 200 & 190 & 180 & 170 & 160 & 150 & 140 & 130 & 120 & 110 & 100 & 90 & 80 & 70 & 60 & 50 & 40 & 30 & 20 & 10 & 0\end{array}$ 
${ }^{1} \mathrm{H}-\mathrm{NMR}$ (400 MHz) spectrum of compound $3 \mathrm{bg}$ in $\mathrm{CDCl}_{3}$

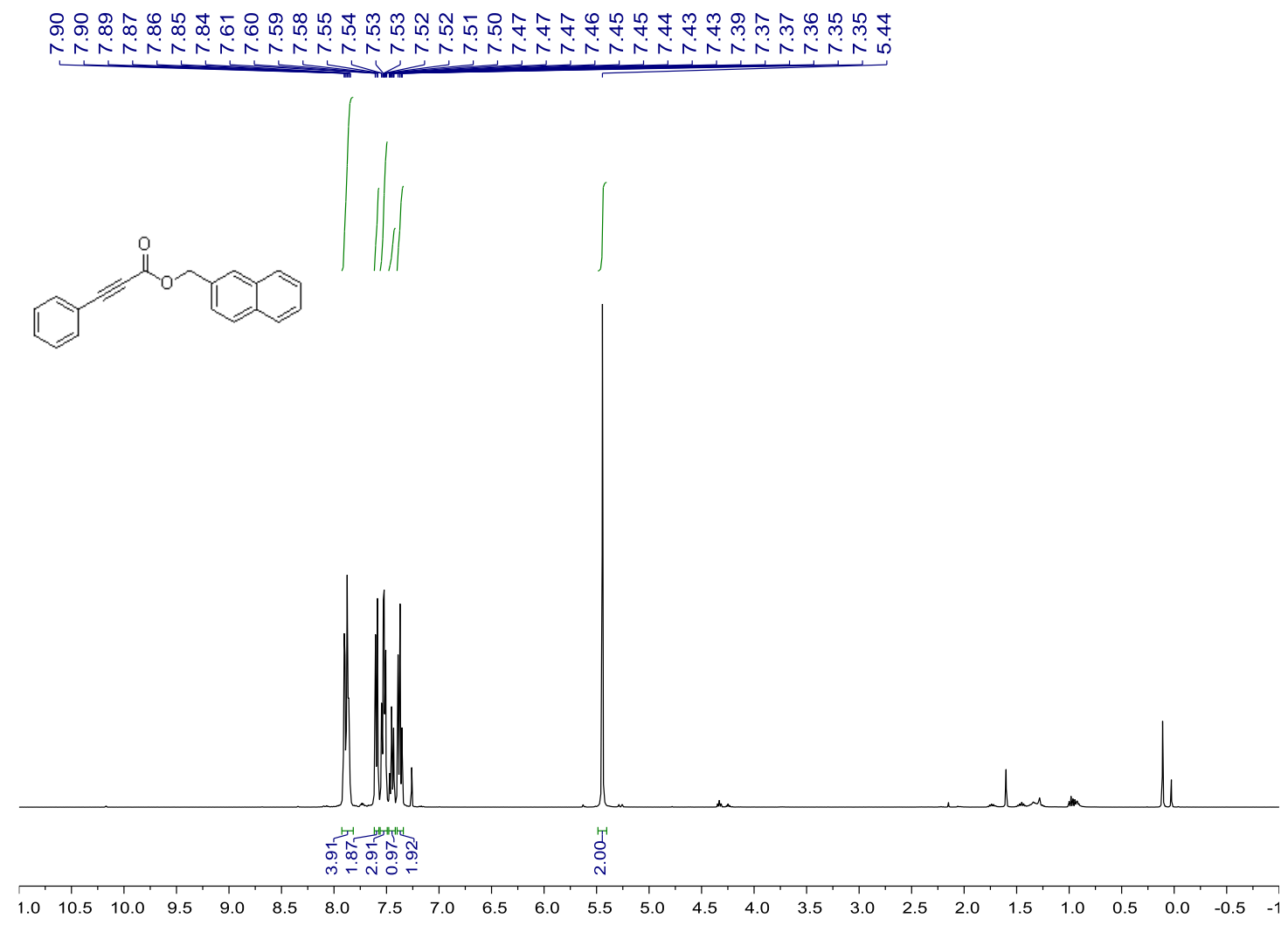

${ }^{13} \mathrm{C}\left\{{ }^{1} \mathrm{H}\right\}$-NMR (100 MHz) spectrum of compound $3 \mathbf{b g}$ in $\mathrm{CDCl}_{3}$
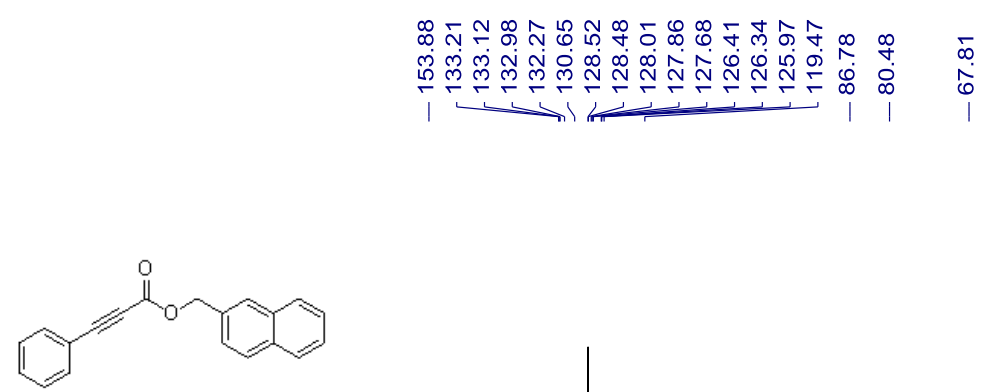

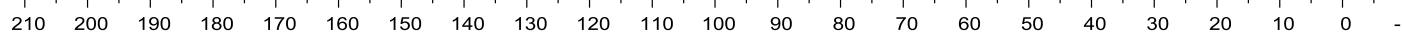


${ }^{1} \mathrm{H}-\mathrm{NMR}(400 \mathrm{MHz})$ spectrum of compound $\mathbf{3 b h}$ in $\mathrm{CDCl}_{3}$

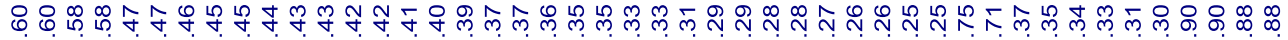

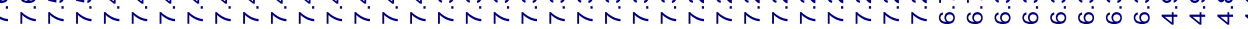
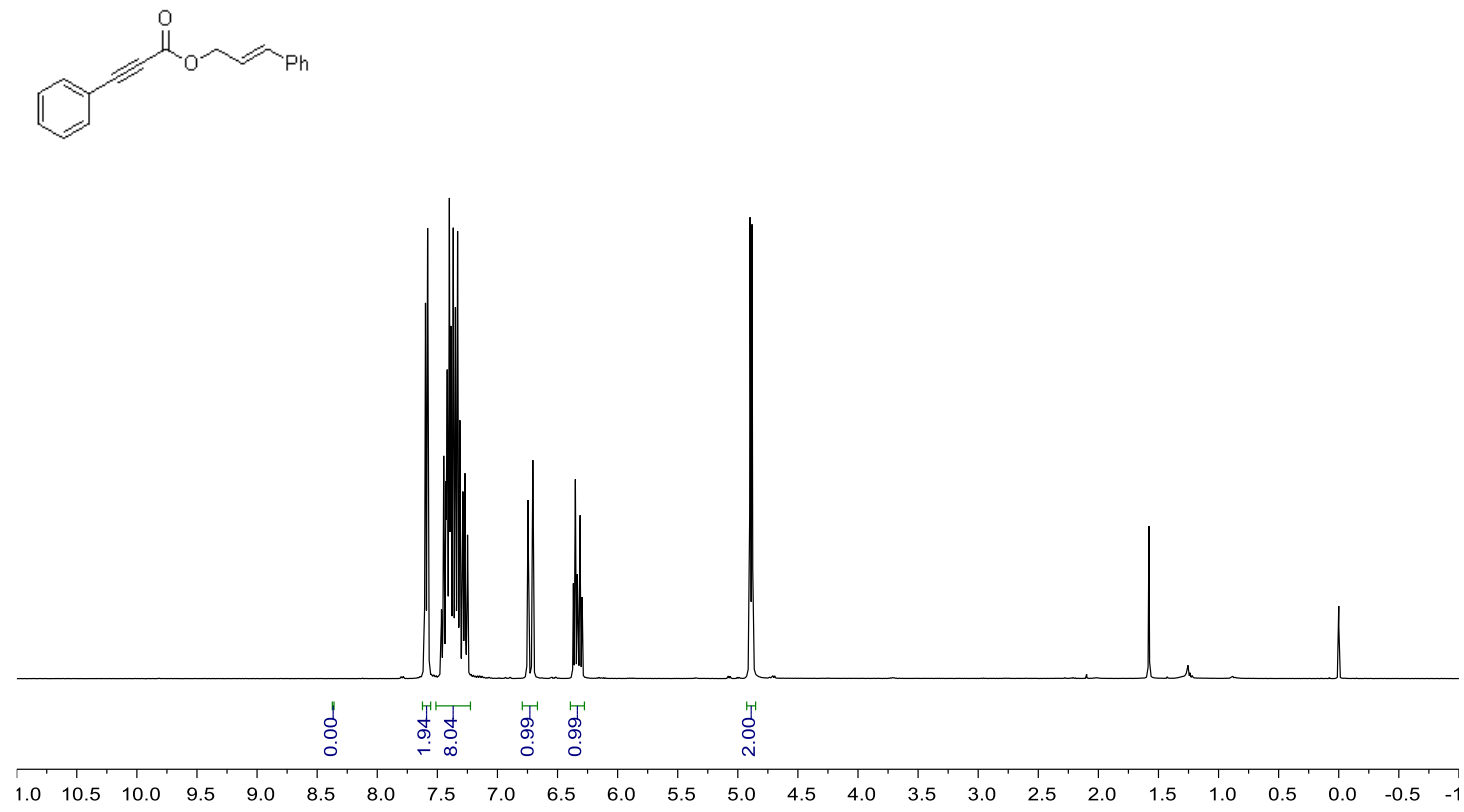

${ }^{13} \mathrm{C}\left\{{ }^{1} \mathrm{H}\right\}$-NMR (100 MHz) spectrum of compound $3 \mathbf{b h}$ in $\mathrm{CDCl}_{3}$
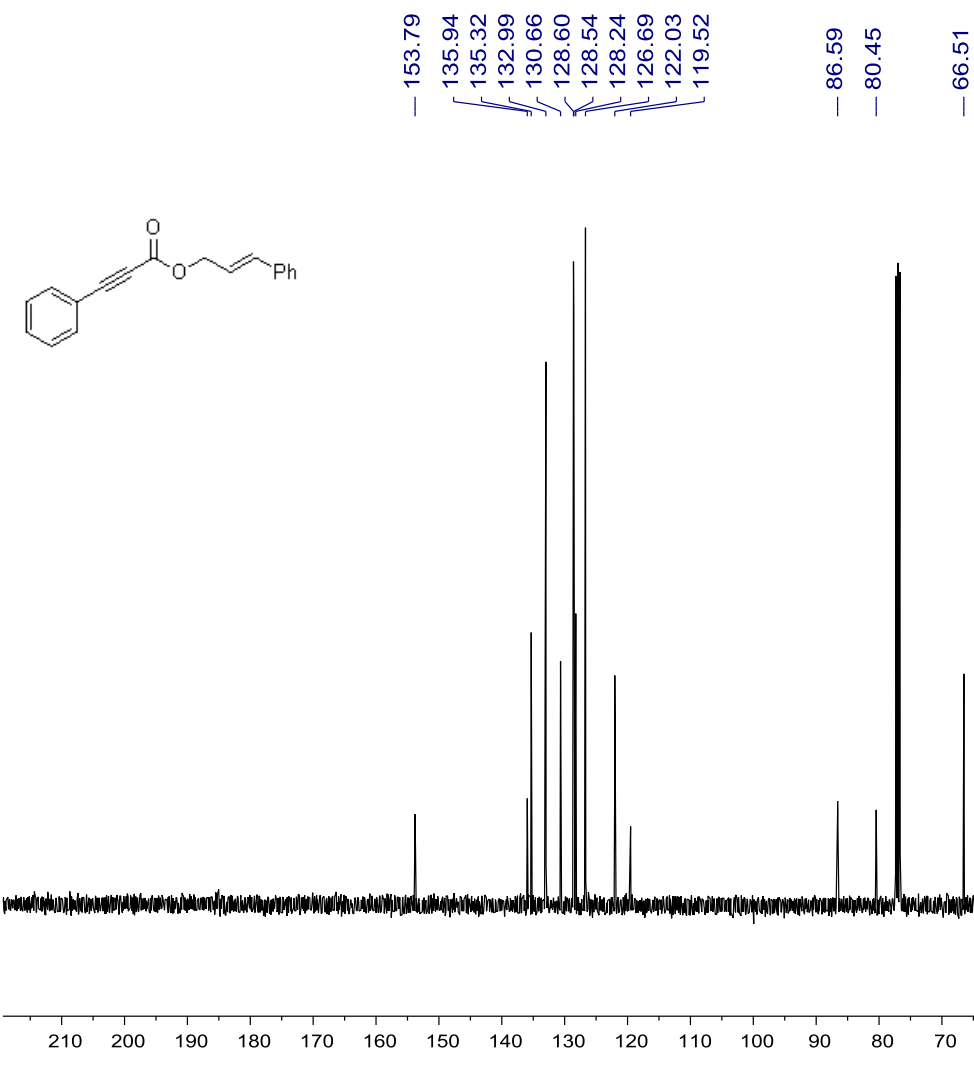
${ }^{1} \mathrm{H}-\mathrm{NMR}(400 \mathrm{MHz})$ spectrum of compound $3 \mathbf{b i}$ in $\mathrm{CDCl}_{3}$

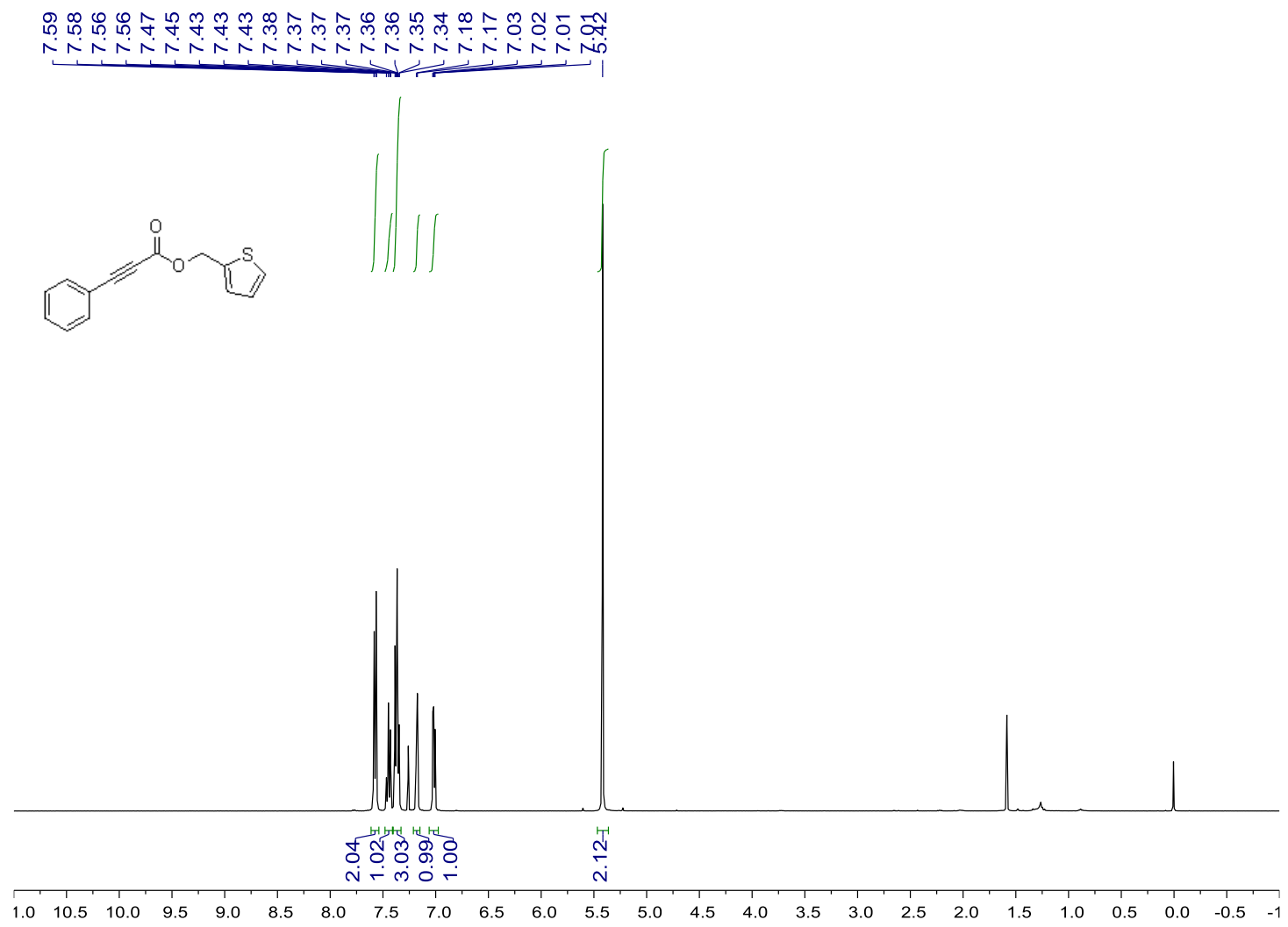

${ }^{13} \mathrm{C}\left\{{ }^{1} \mathrm{H}\right\}$-NMR (100 MHz) spectrum of compound $\mathbf{3 b i}$ in $\mathrm{CDCl}_{3}$

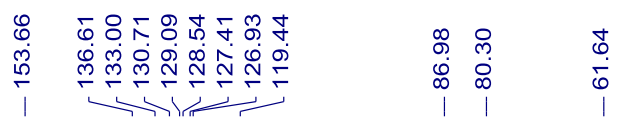<smiles>O=C(COc1ccccc1)c1ccccc1</smiles>

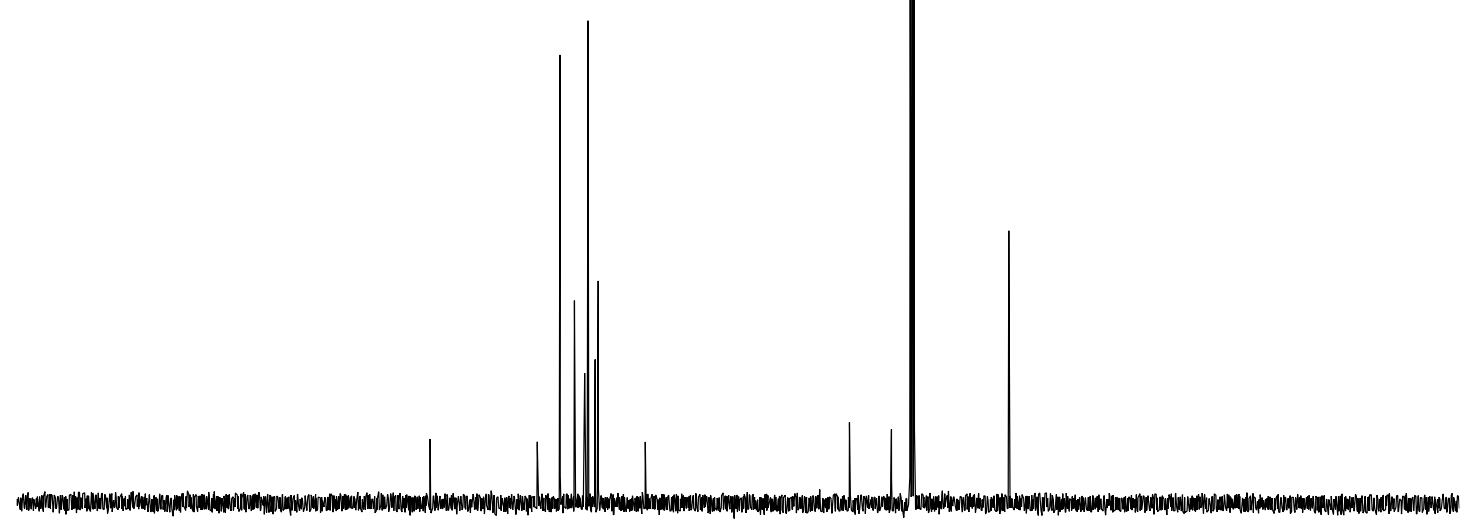

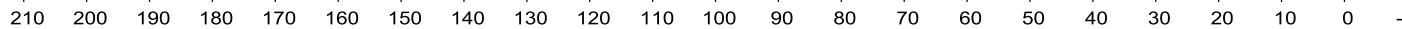


${ }^{1} \mathrm{H}-\mathrm{NMR}$ (400 MHz) spectrum of compound $\mathbf{3 b j}$ in $\mathrm{CDCl}_{3}$

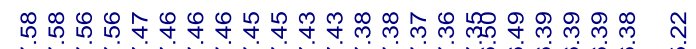

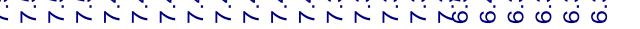
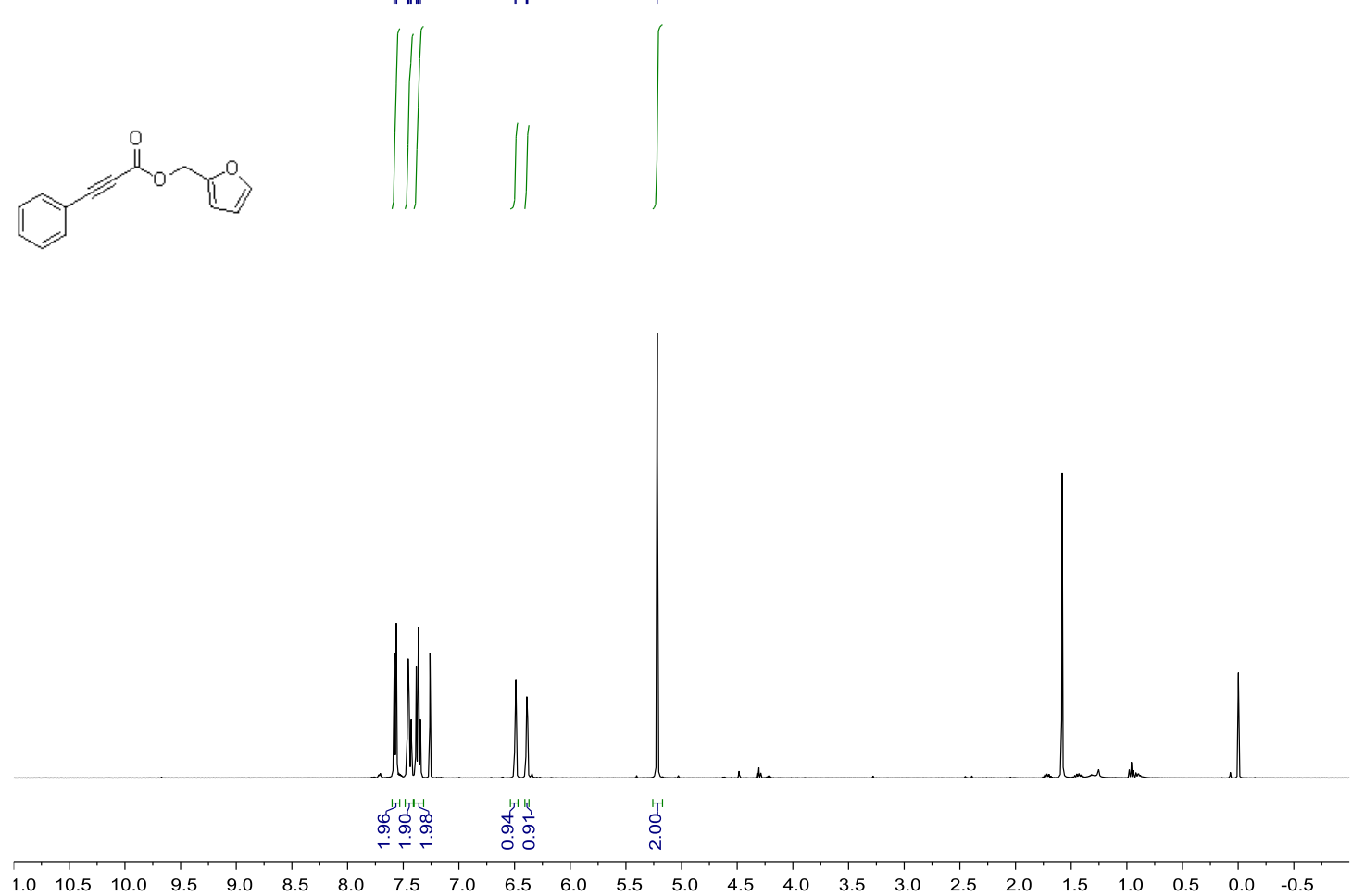

${ }^{13} \mathrm{C}\left\{{ }^{1} \mathrm{H}\right\}$-NMR (100 MHz) spectrum of compound $\mathbf{3 b j}$ in $\mathrm{CDCl}_{3}$

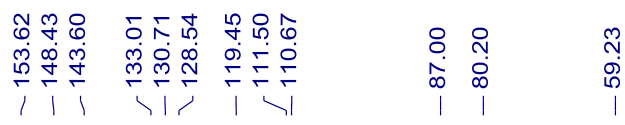

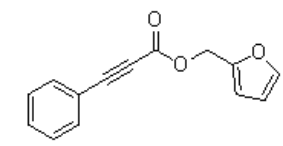

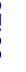


${ }^{1} \mathrm{H}-\mathrm{NMR}\left(400 \mathrm{MHz}\right.$ ) spectrum of compound $\mathbf{3 b \mathbf { k }}$ in $\mathrm{CDCl}_{3}$

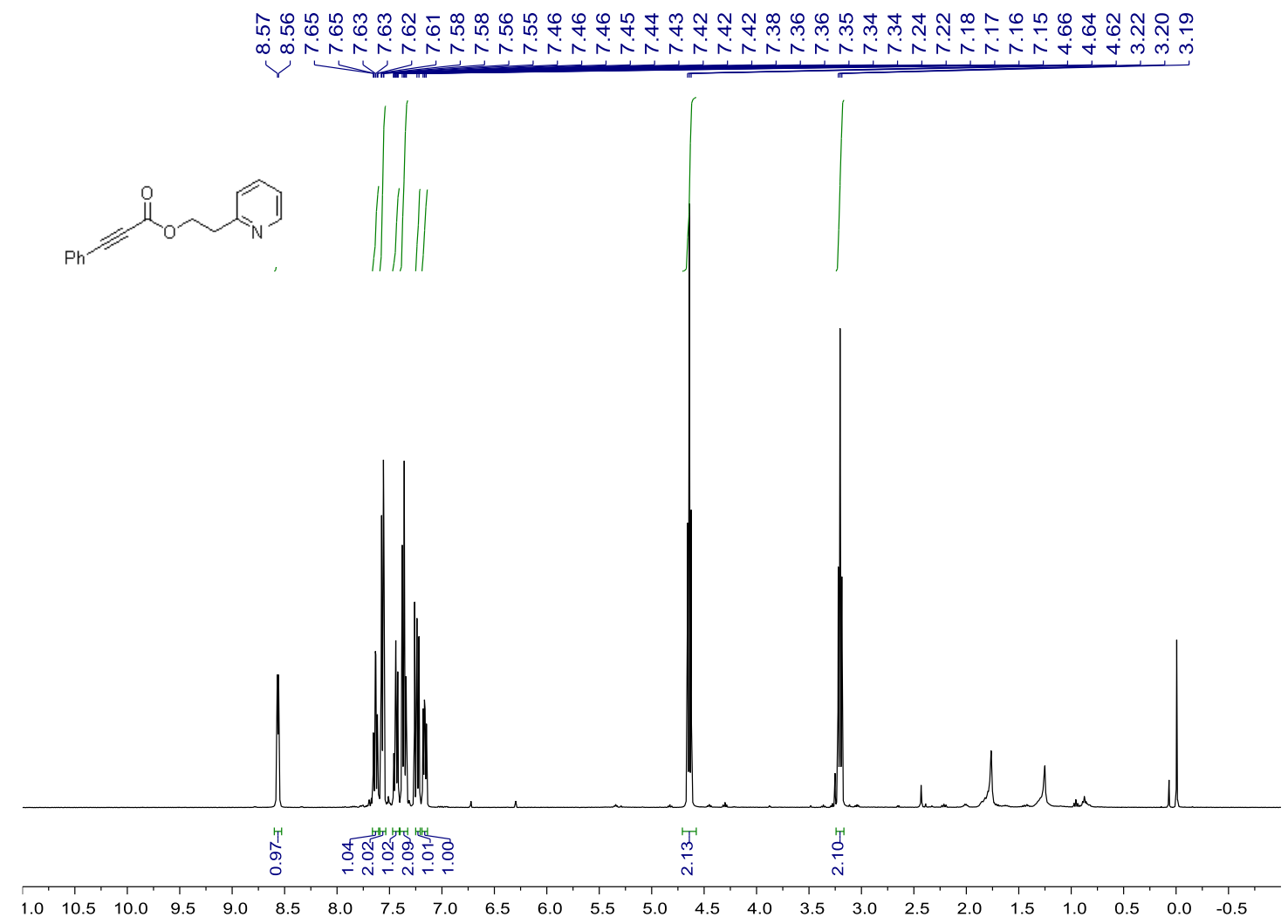

${ }^{13} \mathrm{C}\left\{{ }^{1} \mathrm{H}\right\}-\mathrm{NMR}(100 \mathrm{MHz})$ spectrum of compound $\mathbf{3 b k}$ in $\mathrm{CDCl}_{3}$

\begin{tabular}{|c|c|c|}
\hline 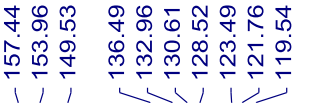 & 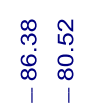 & $\begin{array}{l}0 \\
0 \\
0 \\
0\end{array}$ \\
\hline
\end{tabular}<smiles>O=C(C#CCOCc1ccccn1)OCCc1ccccn1</smiles>

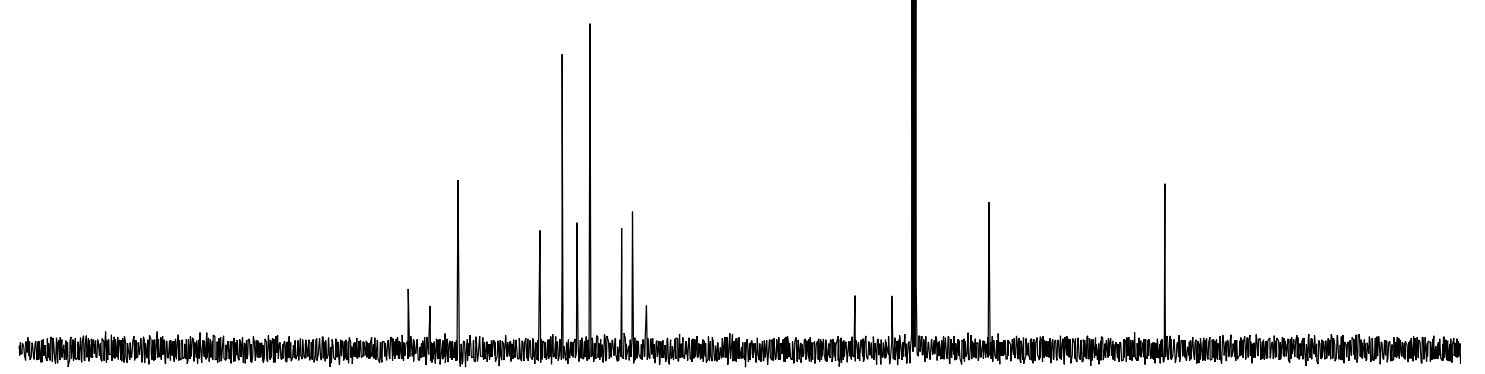

$\begin{array}{llllllllllllllllllllll}210 & 200 & 190 & 180 & 170 & 160 & 150 & 140 & 130 & 120 & 110 & 100 & 90 & 80 & 70 & 60 & 50 & 40 & 30 & 20 & 10 & 0\end{array}$. 
${ }^{1} \mathrm{H}-\mathrm{NMR}\left(400 \mathrm{MHz}\right.$ ) spectrum of compound $3 \mathbf{b l}$ in $\mathrm{CDCl}_{3}$

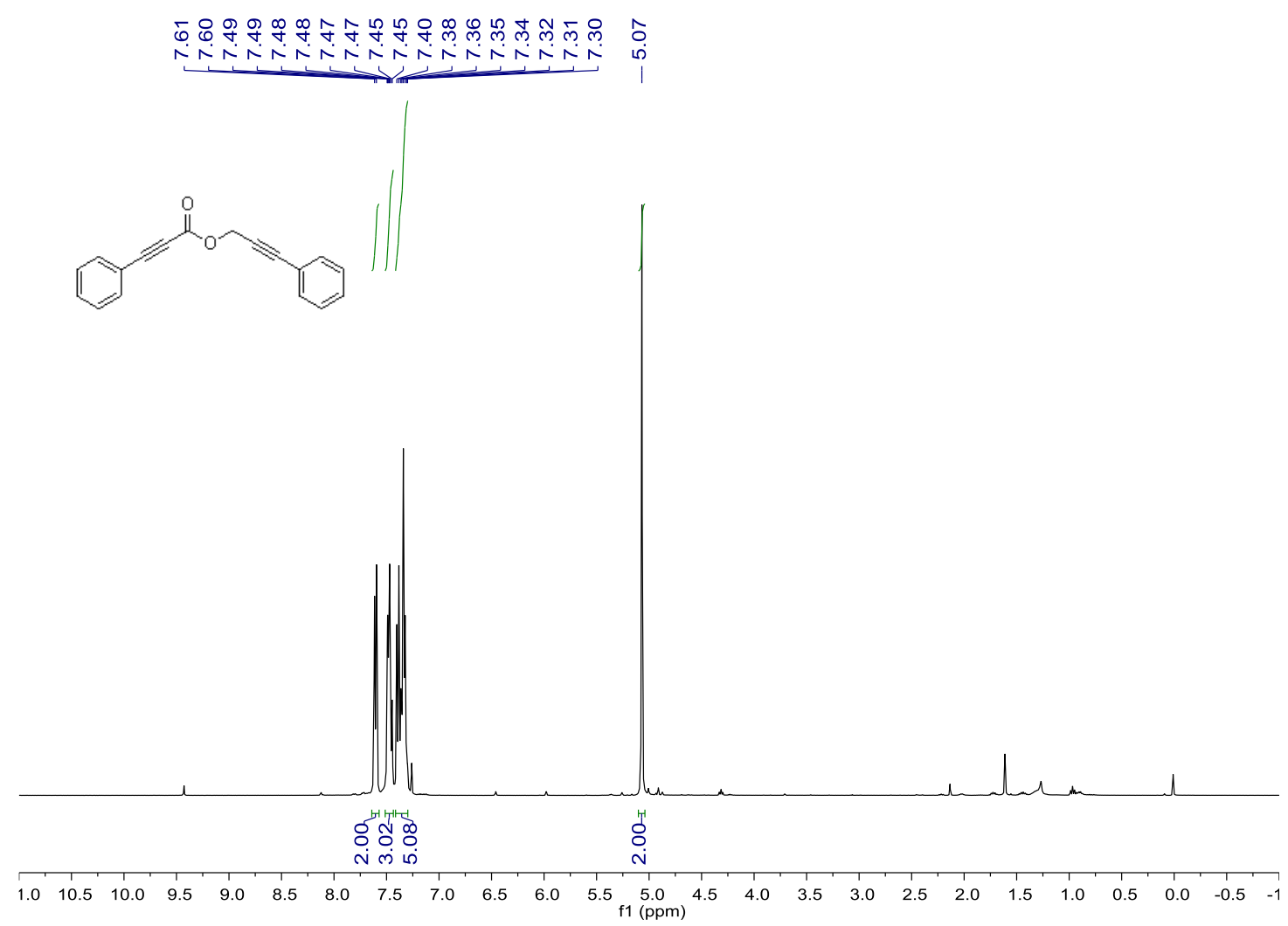

${ }^{13} \mathrm{C}\left\{{ }^{1} \mathrm{H}\right\}$-NMR (100 MHz) spectrum of compound $\mathbf{3 b l}$ in $\mathrm{CDCl}_{3}$

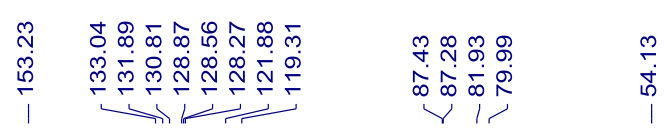
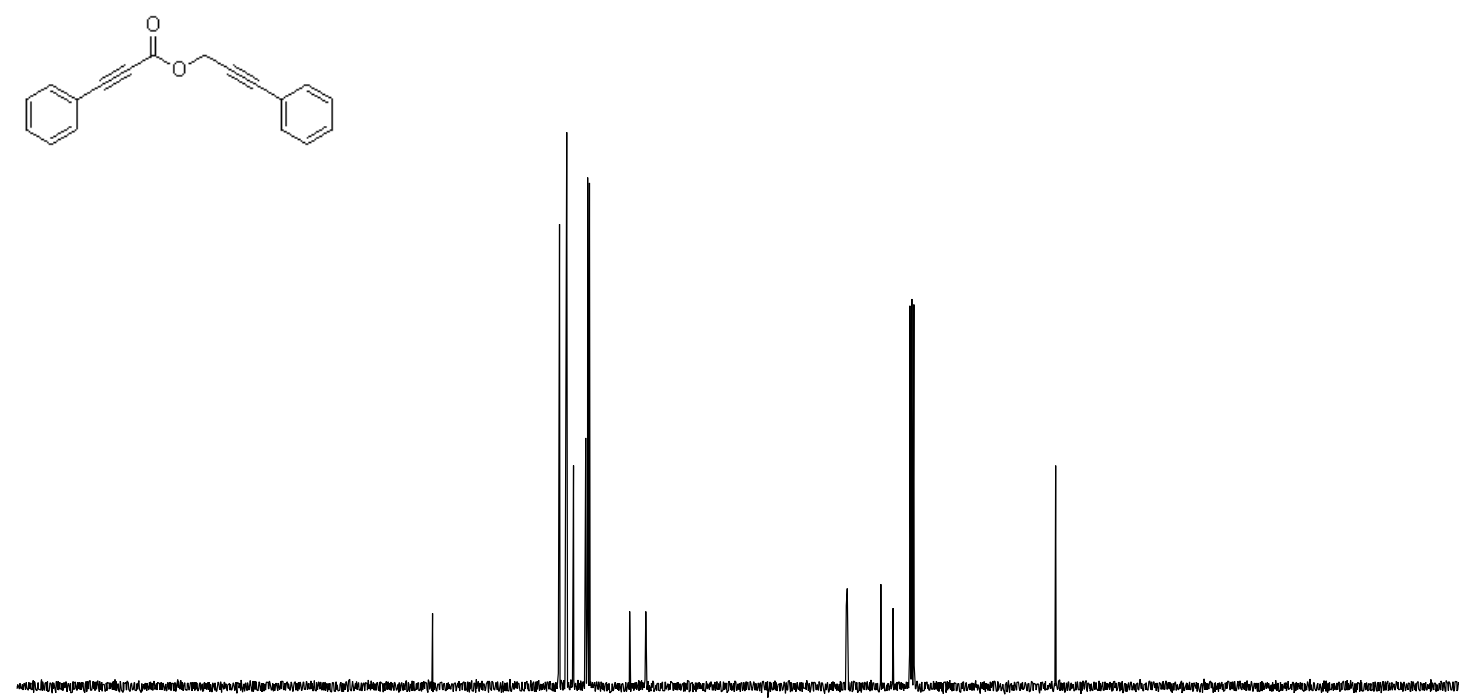

$\begin{array}{lllllllllllllllllllllllllllll}210 & 200 & 190 & 180 & 170 & 160 & 150 & 140 & 130 & 120 & 110 & 100 & 90 & 80 & 70 & 60 & 50 & 40 & 30 & 20 & 10 & 0 & -\end{array}$ 
${ }^{1} \mathrm{H}-\mathrm{NMR}\left(400 \mathrm{MHz}\right.$ ) spectrum of compound $\mathbf{3 b m}$ in $\mathrm{CDCl}_{3}$

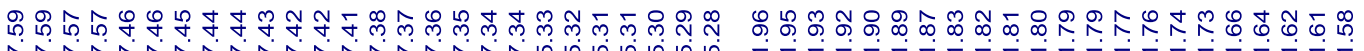

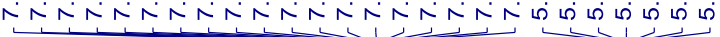
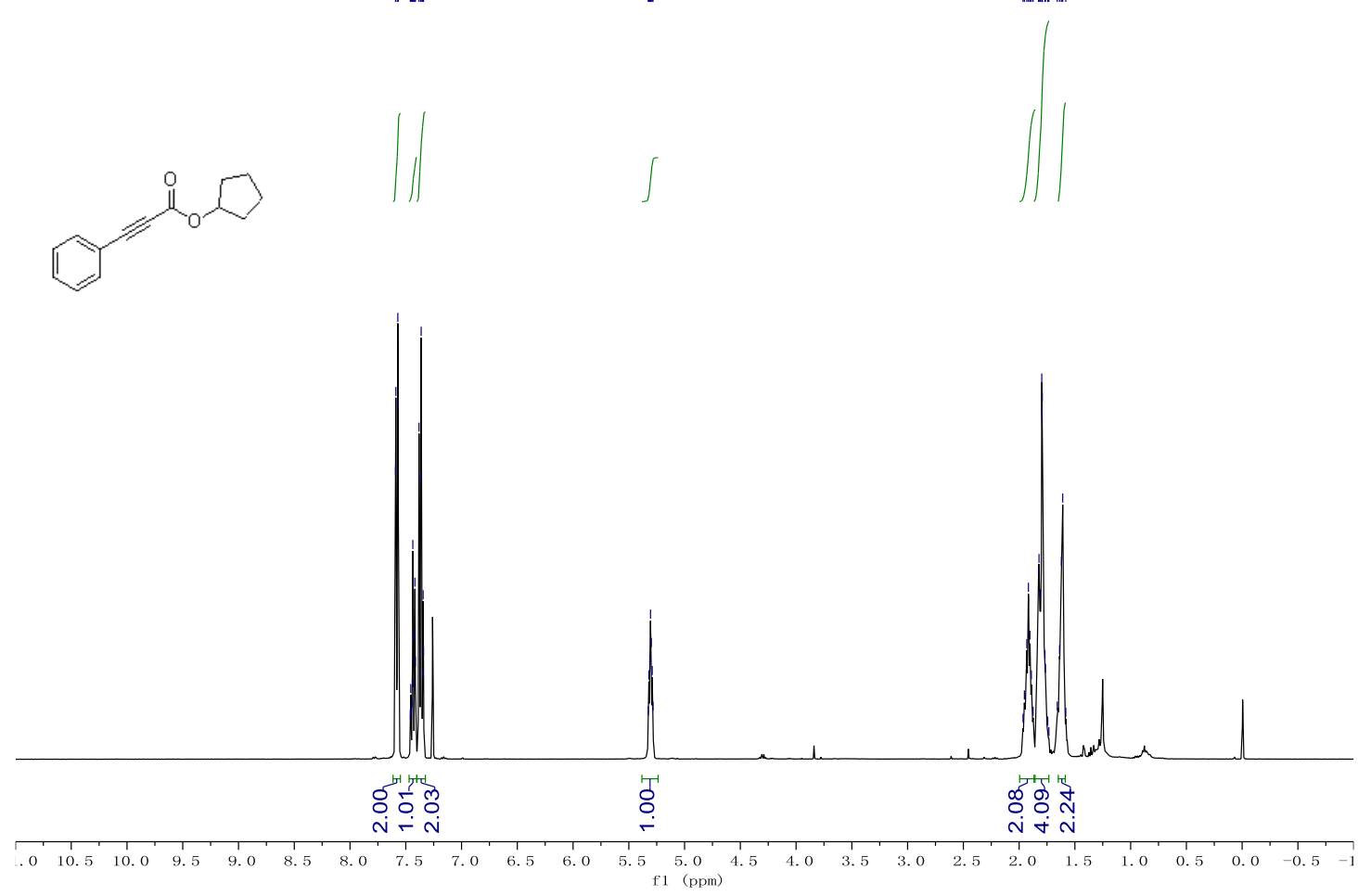

${ }^{13} \mathrm{C}\left\{{ }^{1} \mathrm{H}\right\}$-NMR (100 MHz) spectrum of compound $\mathbf{3 b m}$ in $\mathrm{CDCl}_{3}$

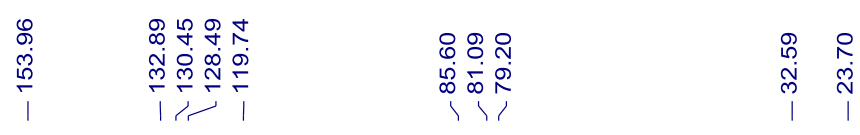
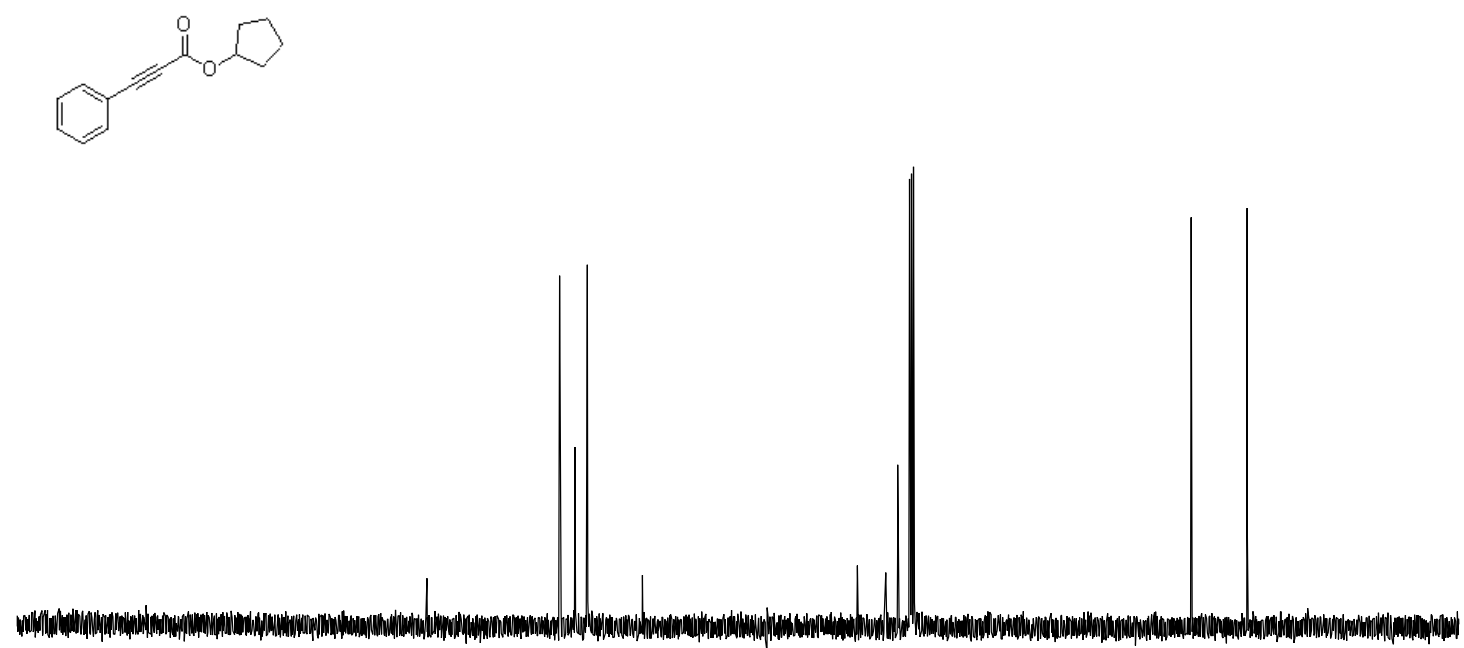
${ }^{1} \mathrm{H}-\mathrm{NMR}$ (400 MHz) spectrum of compound $\mathbf{3 b n}$ in $\mathrm{CDCl}_{3}$

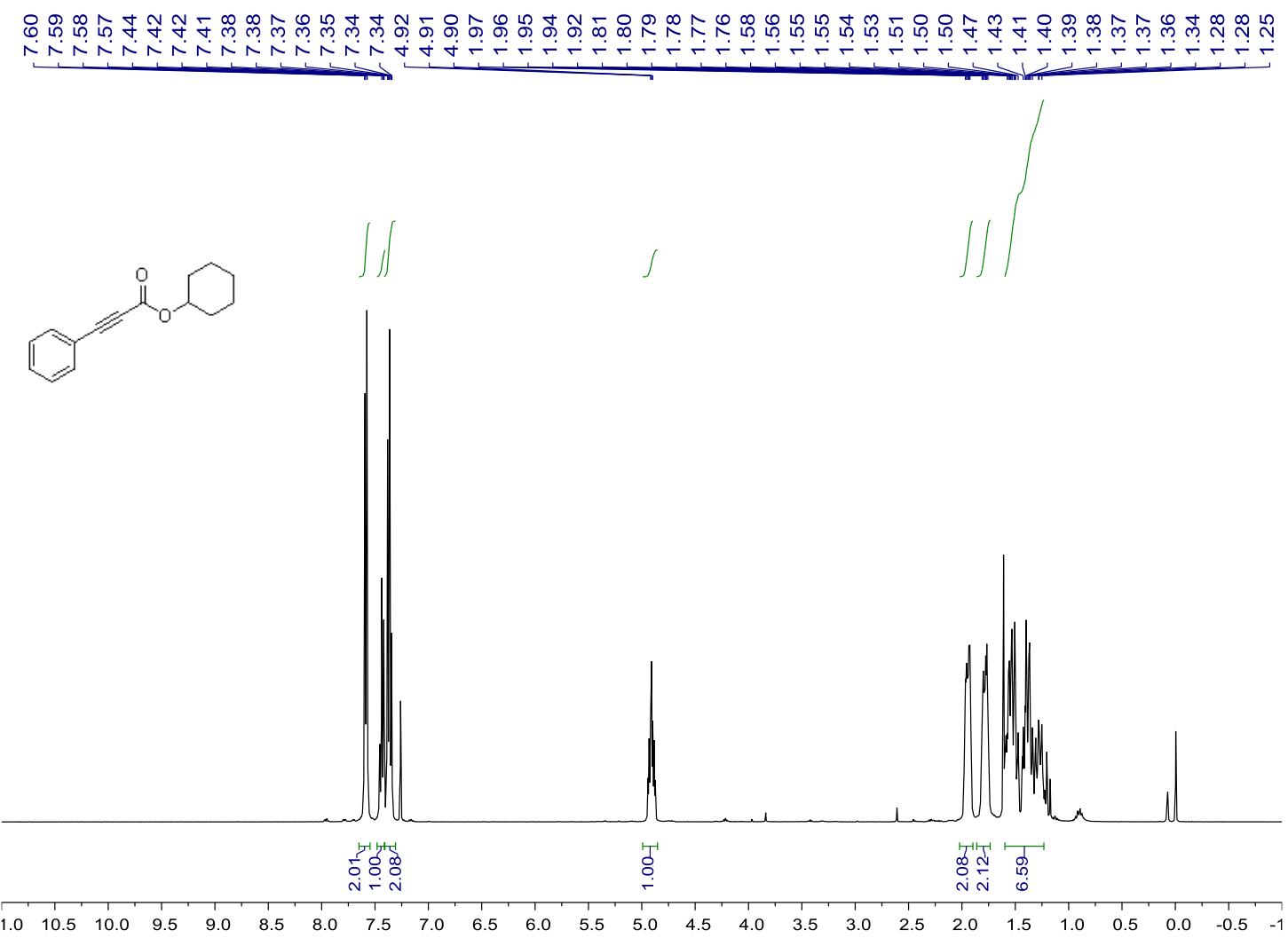

${ }^{13} \mathrm{C}\left\{{ }^{1} \mathrm{H}\right\}-\mathrm{NMR}(100 \mathrm{MHz})$ spectrum of compound $3 \mathbf{b n}$ in $\mathrm{CDCl}_{3}$

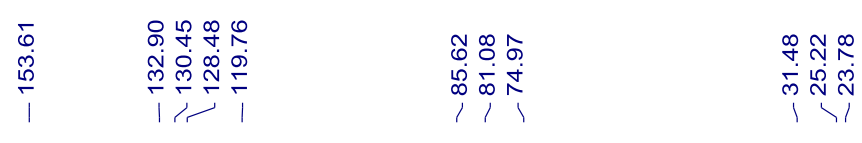

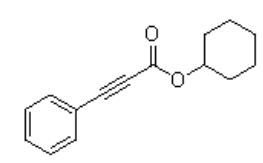

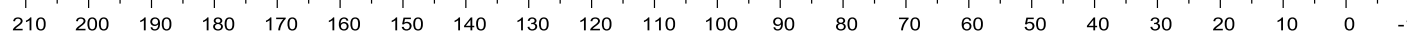


${ }^{1} \mathrm{H}-\mathrm{NMR}(400 \mathrm{MHz})$ spectrum of compound $\mathbf{6 a}$ in $\mathrm{CDCl}_{3}$

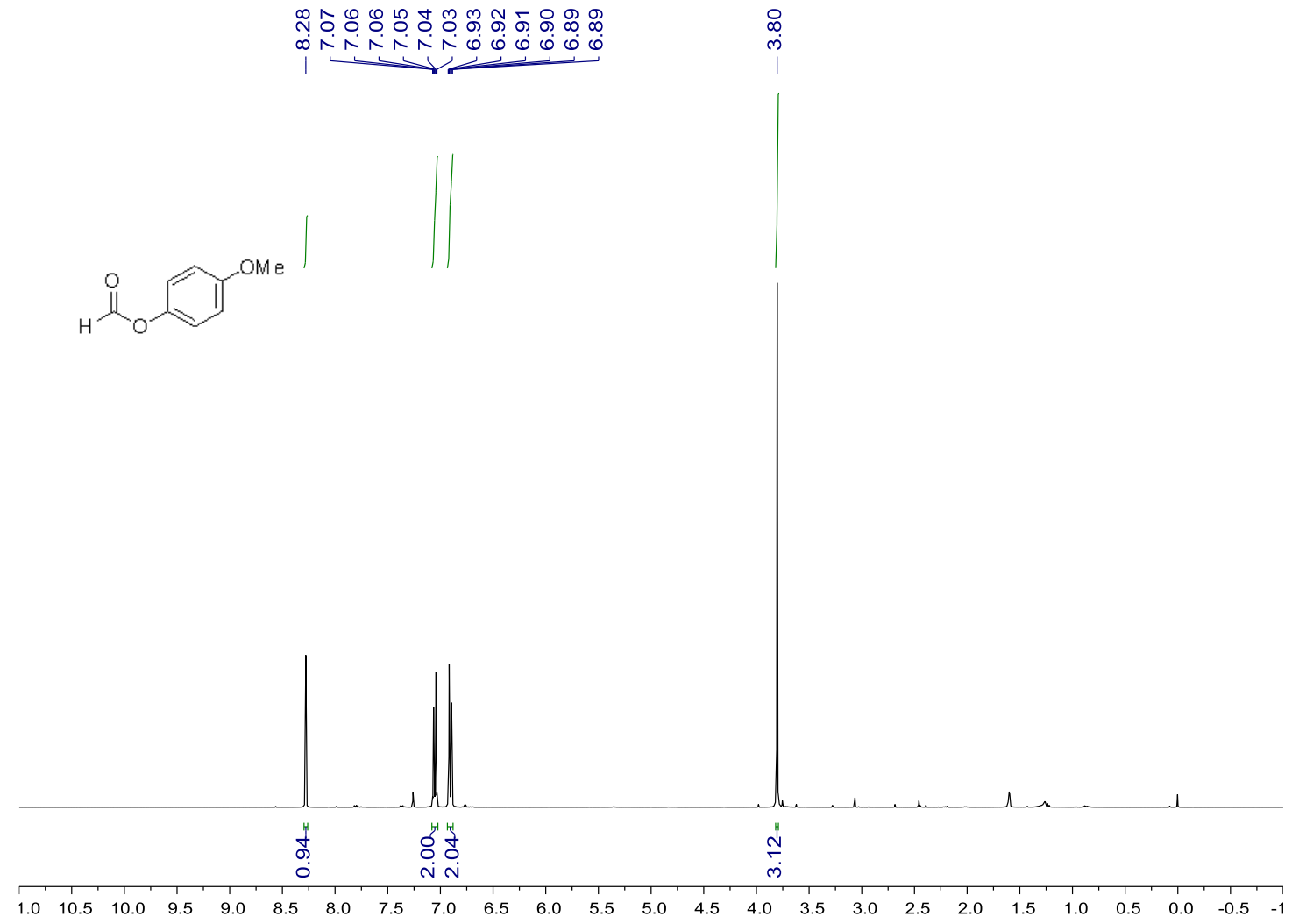

${ }^{13} \mathrm{C}\left\{{ }^{1} \mathrm{H}\right\}$-NMR (100 MHz) spectrum of compound $\mathbf{6 a}$ in $\mathrm{CDCl}_{3}$

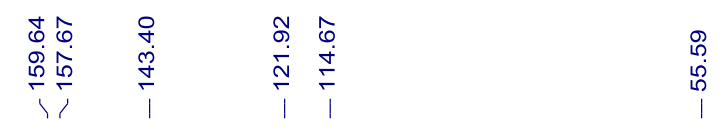
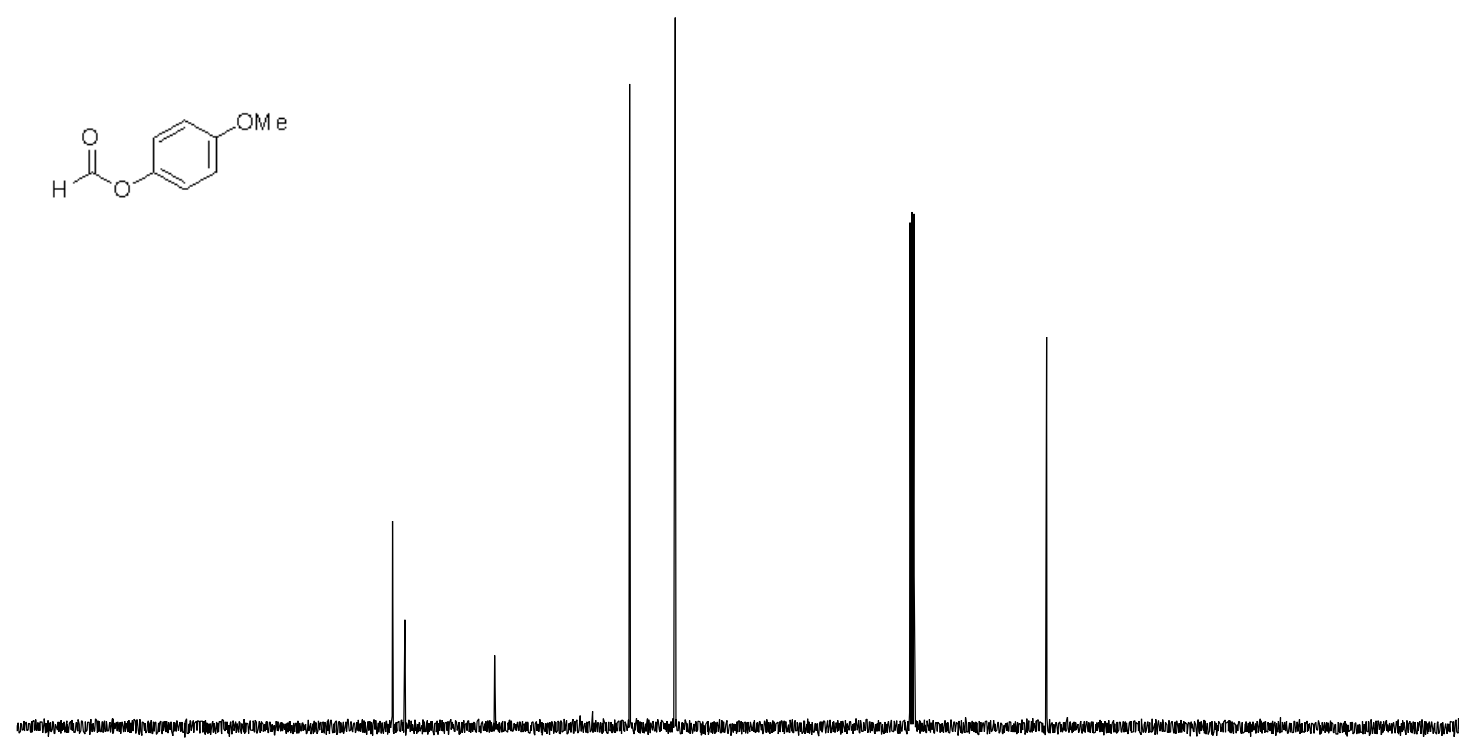

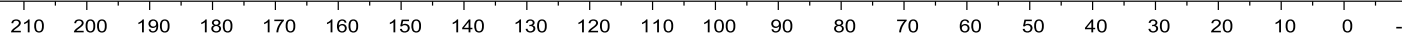


${ }^{1} \mathrm{H}-\mathrm{NMR}(400 \mathrm{MHz})$ spectrum of compound $\mathbf{6 b}$ in $\mathrm{CDCl}_{3}$
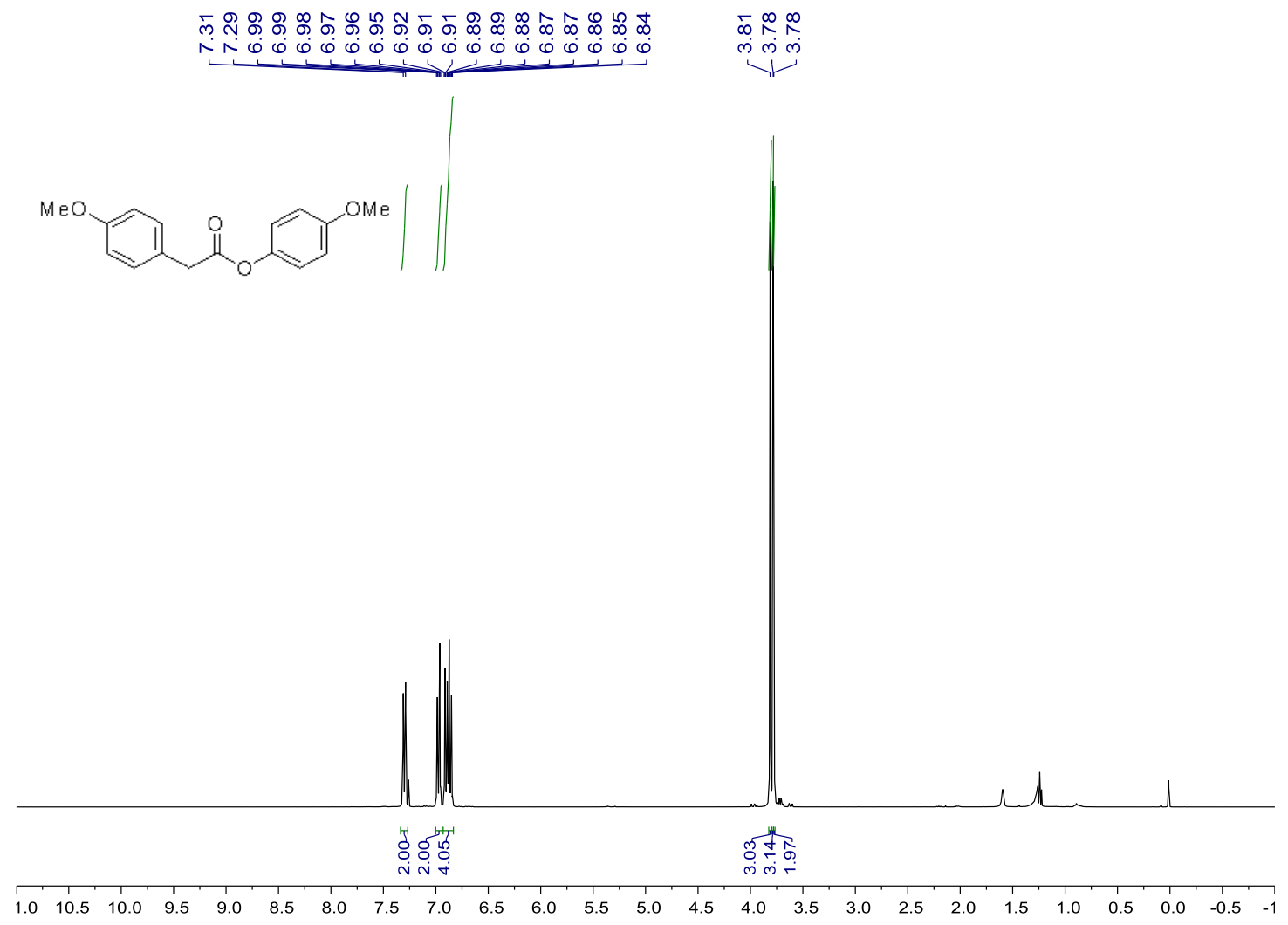

${ }^{13} \mathrm{C}\left\{{ }^{1} \mathrm{H}\right\}$-NMR $(100 \mathrm{MHz})$ spectrum of compound $\mathbf{6 b}$ in $\mathrm{CDCl}_{3}$

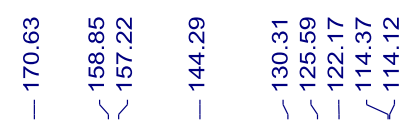

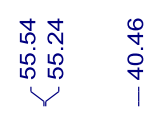
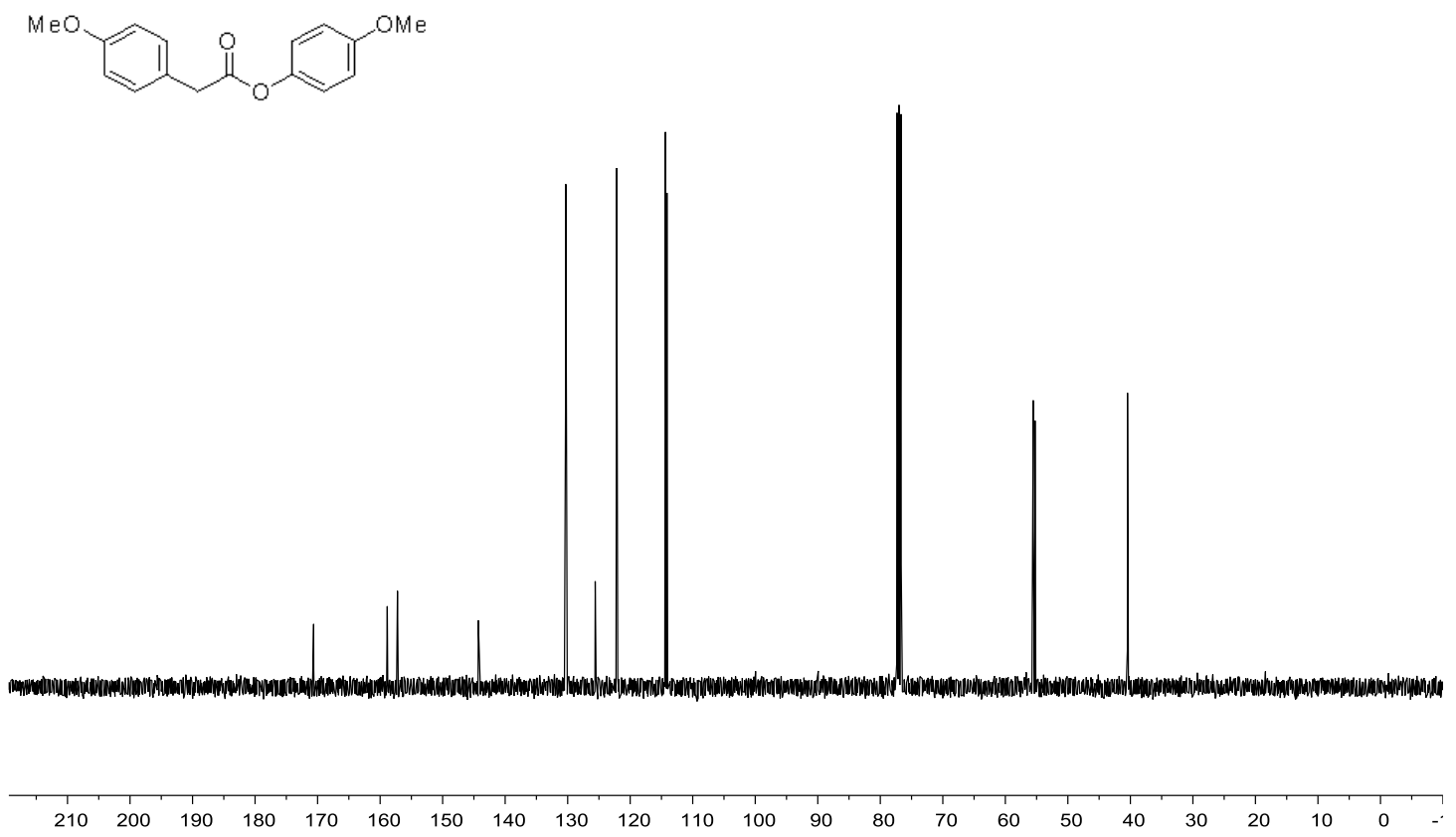

SI-35 
${ }^{1} \mathrm{H}-\mathrm{NMR}\left(400 \mathrm{MHz}\right.$ ) spectrum of compound $\mathbf{6} \mathbf{c}$ in $\mathrm{CDCl}_{3}$

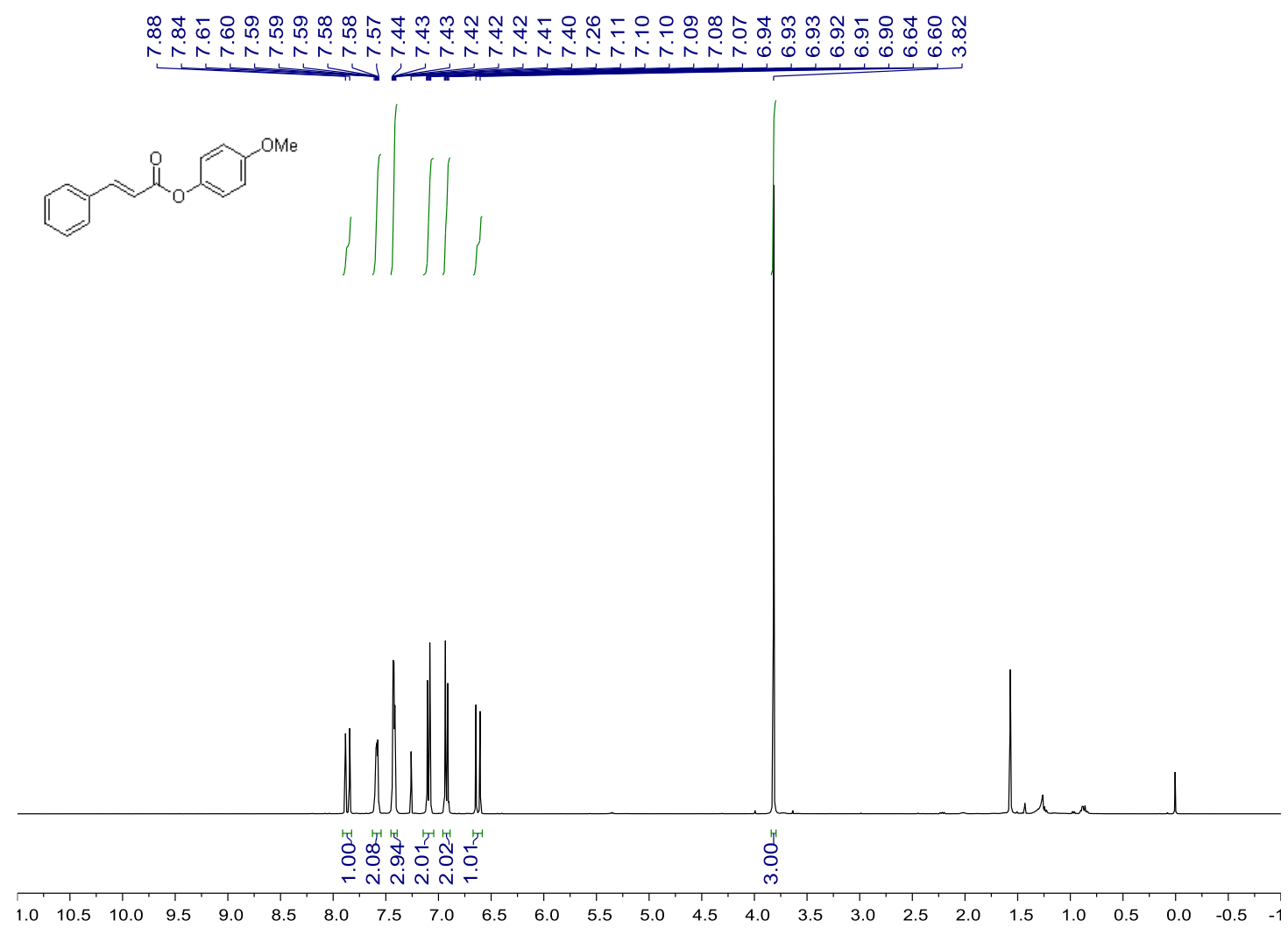

${ }^{13} \mathrm{C}\left\{{ }^{1} \mathrm{H}\right\}$-NMR (100 MHz) spectrum of compound $\mathbf{6 c}$ in $\mathrm{CDCl}_{3}$

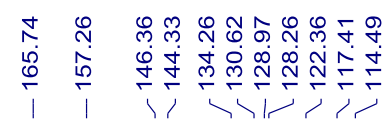

$\stackrel{8}{8}$

i⿱宀㠯
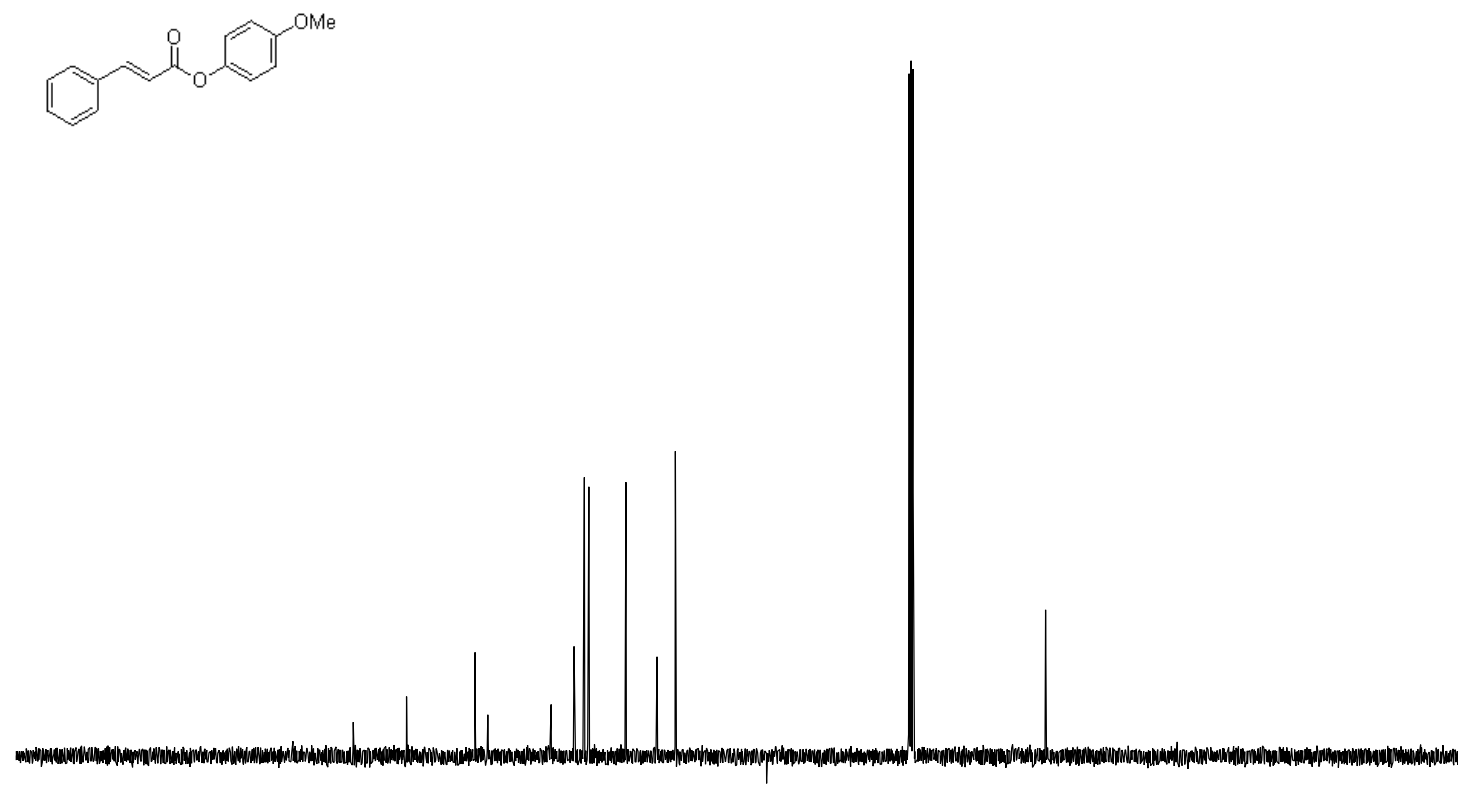

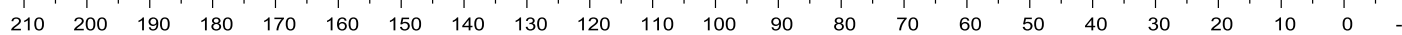


${ }^{1} \mathrm{H}-\mathrm{NMR}(400 \mathrm{MHz})$ spectrum of compound $\mathbf{6 d}$ in $\mathrm{CDCl}_{3}$

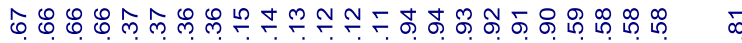

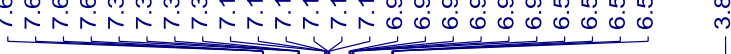
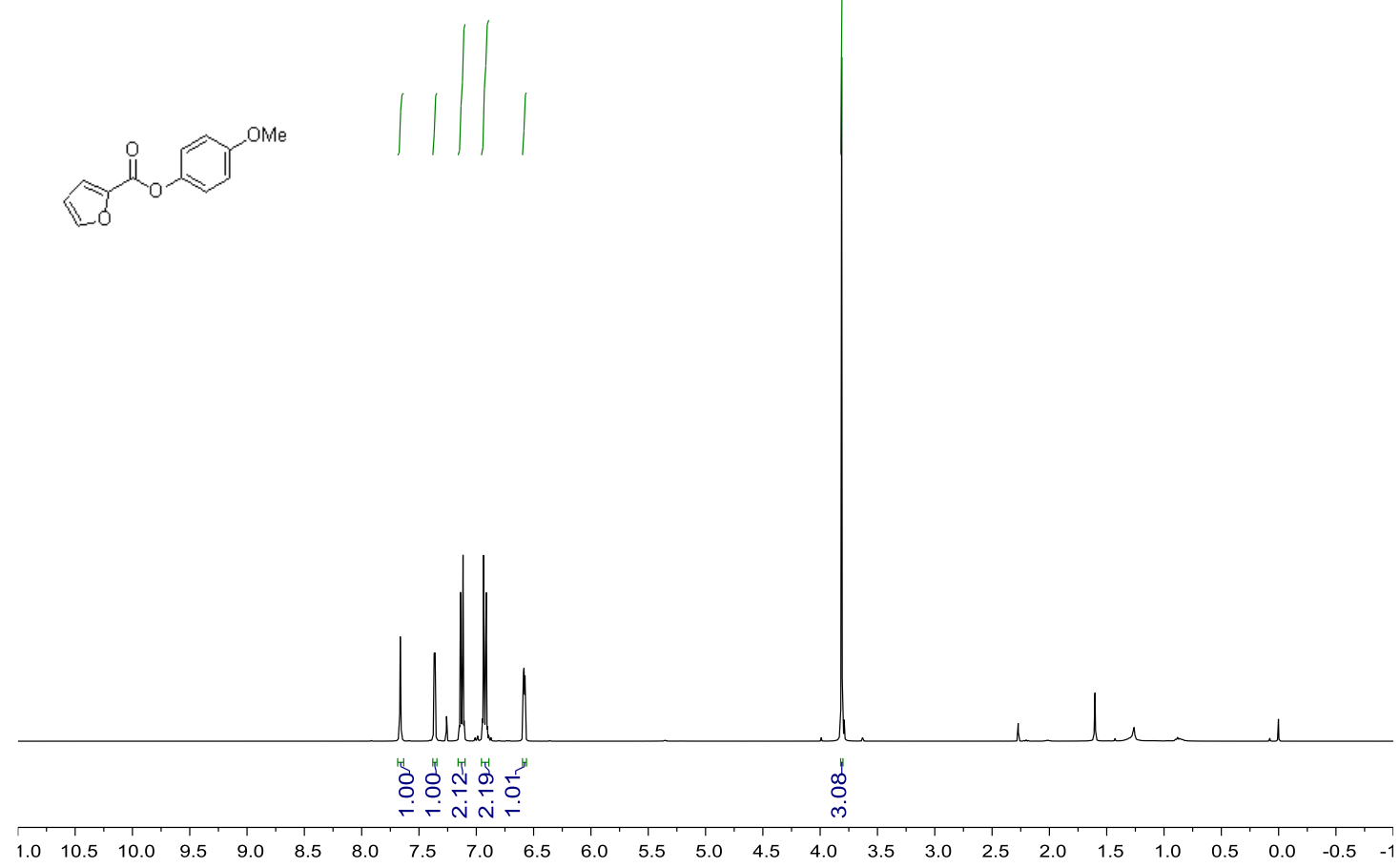

${ }^{13} \mathrm{C}\left\{{ }^{1} \mathrm{H}\right\}$-NMR (100 MHz) spectrum of compound $\mathbf{6 d}$ in $\mathrm{CDCl}_{3}$

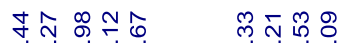

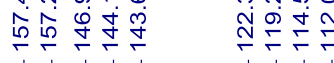

苜

$\checkmark>Y \quad \mid 11$
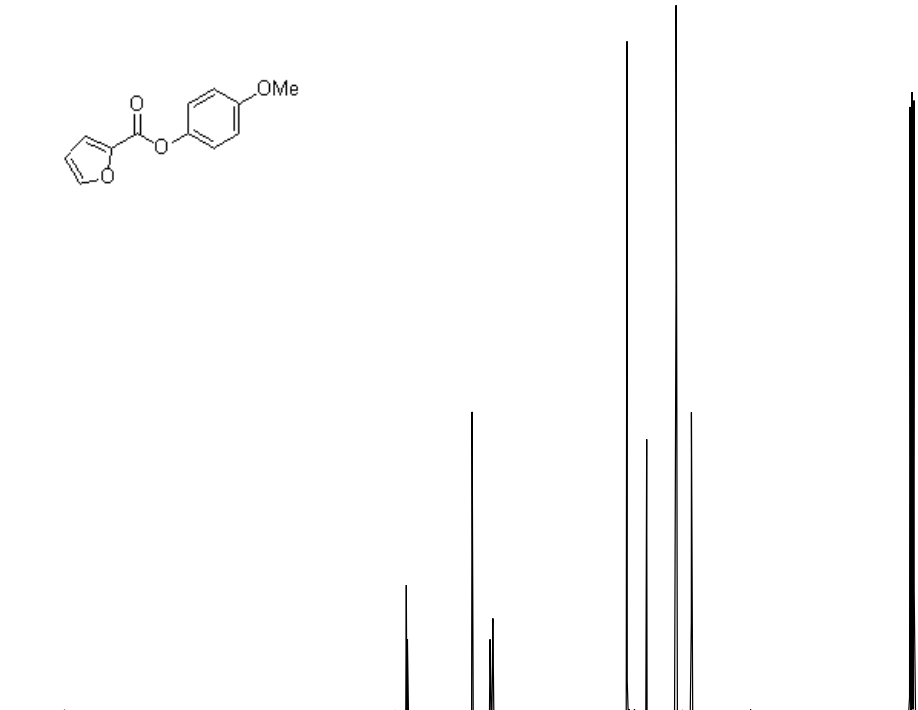

$\begin{array}{llllllllll}210 & 200 & 190 & 180 & 170 & 160 & 150 & 140 & 130 & 120\end{array}$

110100

$90 \quad 80$

60 
${ }^{1} \mathrm{H}-\mathrm{NMR}(400 \mathrm{MHz})$ spectrum of compound $\mathbf{6 e}$ in $\mathrm{CDCl}_{3}$

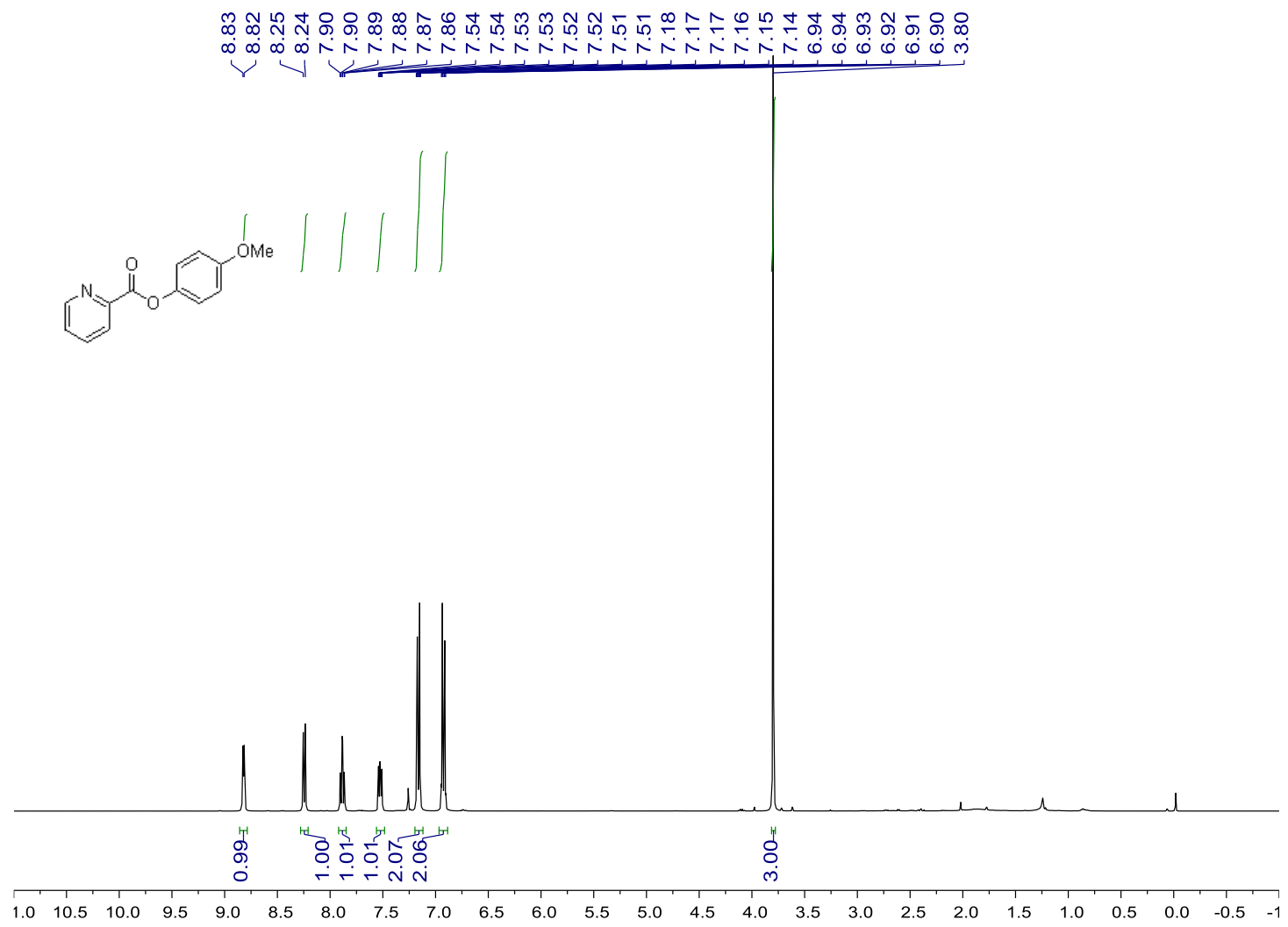

${ }^{13} \mathrm{C}\left\{{ }^{1} \mathrm{H}\right\}$-NMR (100 MHz) spectrum of compound $\mathbf{6 e}$ in $\mathrm{CDCl}_{3}$

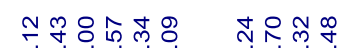

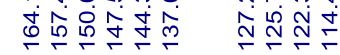

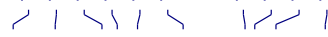

in
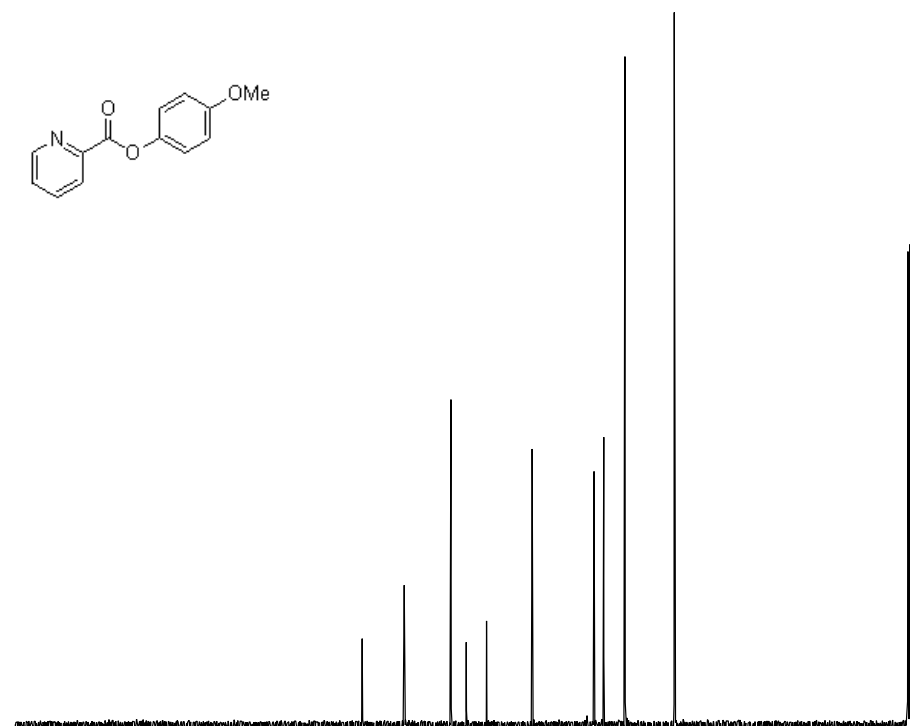

$210 \quad 200$

$190 \quad 180$

$\begin{array}{lll}70 & 160 & 150\end{array}$

$130 \quad 120$

$110 \quad 100$

$90 \quad 80$

60

$50 \quad 40 \quad 30$

20 
${ }^{1} \mathrm{H}-\mathrm{NMR}\left(400 \mathrm{MHz}\right.$ ) spectrum of compound $\mathbf{6} \mathbf{f}$ in $\mathrm{CDCl}_{3}$

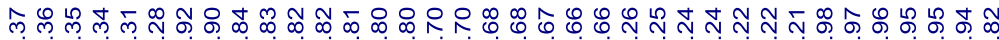

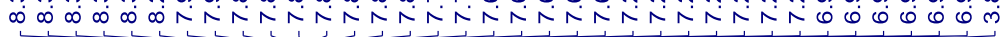
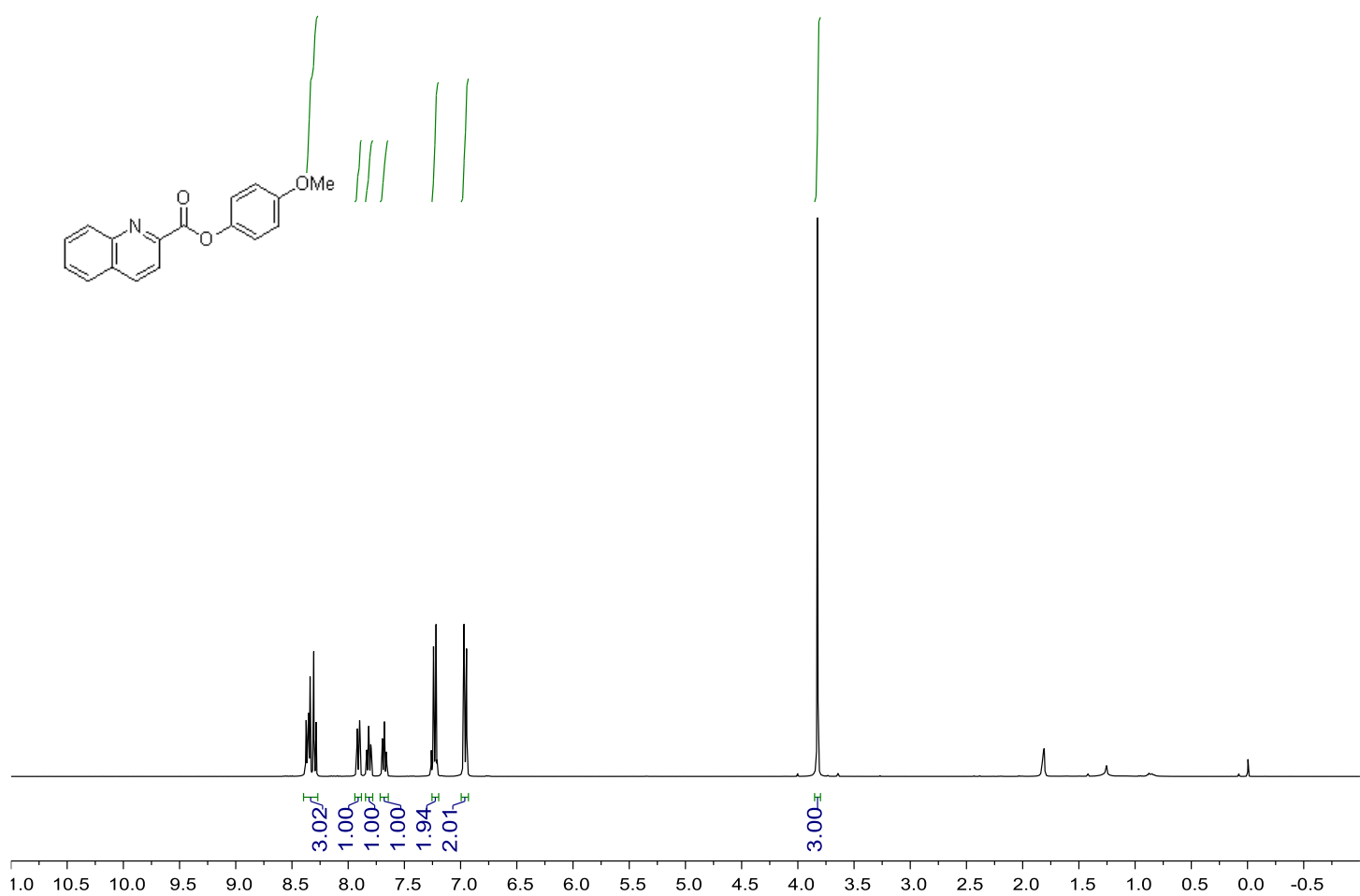

${ }^{13} \mathrm{C}\left\{{ }^{1} \mathrm{H}\right\}$-NMR (100 MHz) spectrum of compound $\mathbf{6} \mathbf{f}$ in $\mathrm{CDCl}_{3}$

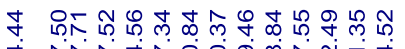

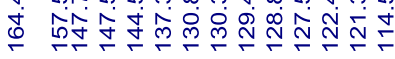

เి

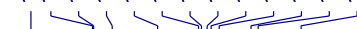

เू
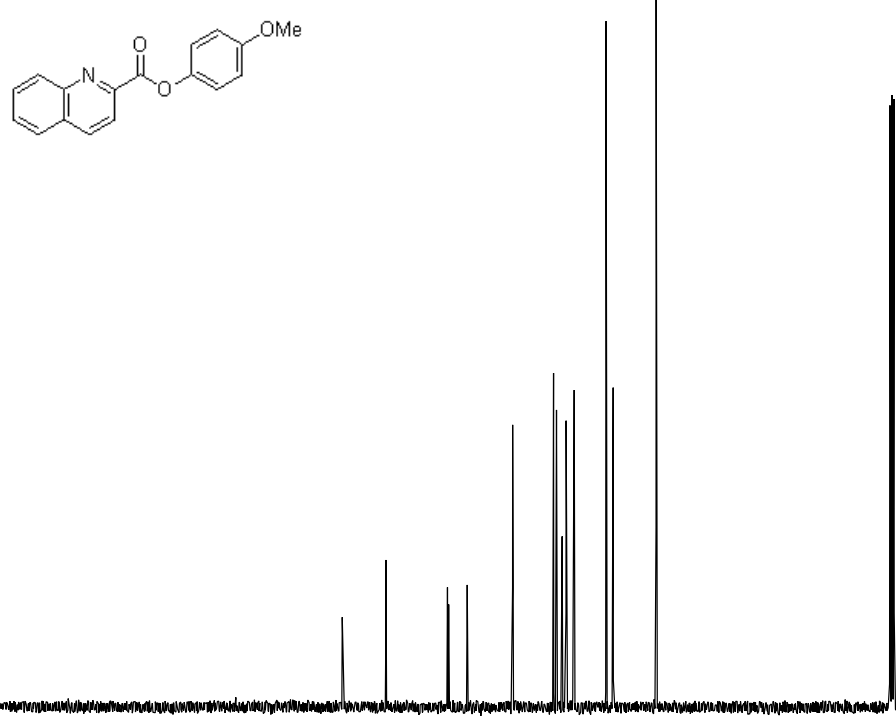

$\begin{array}{llllllllllll}210 & 200 & 190 & 180 & 170 & 160 & 150 & 140 & 130 & 120 & 110 & 100\end{array}$

$90 \quad 80$

$70 \quad 60$

$50 \quad 40$

(1) 
${ }^{1} \mathrm{H}-\mathrm{NMR}(400 \mathrm{MHz})$ spectrum of compound $\mathbf{6} \mathbf{g}$ in $\mathrm{CDCl}_{3}$
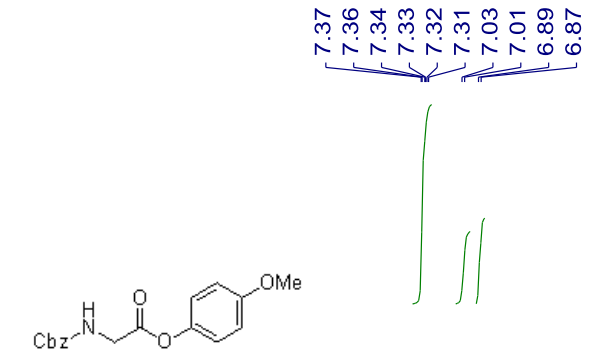

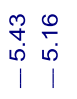
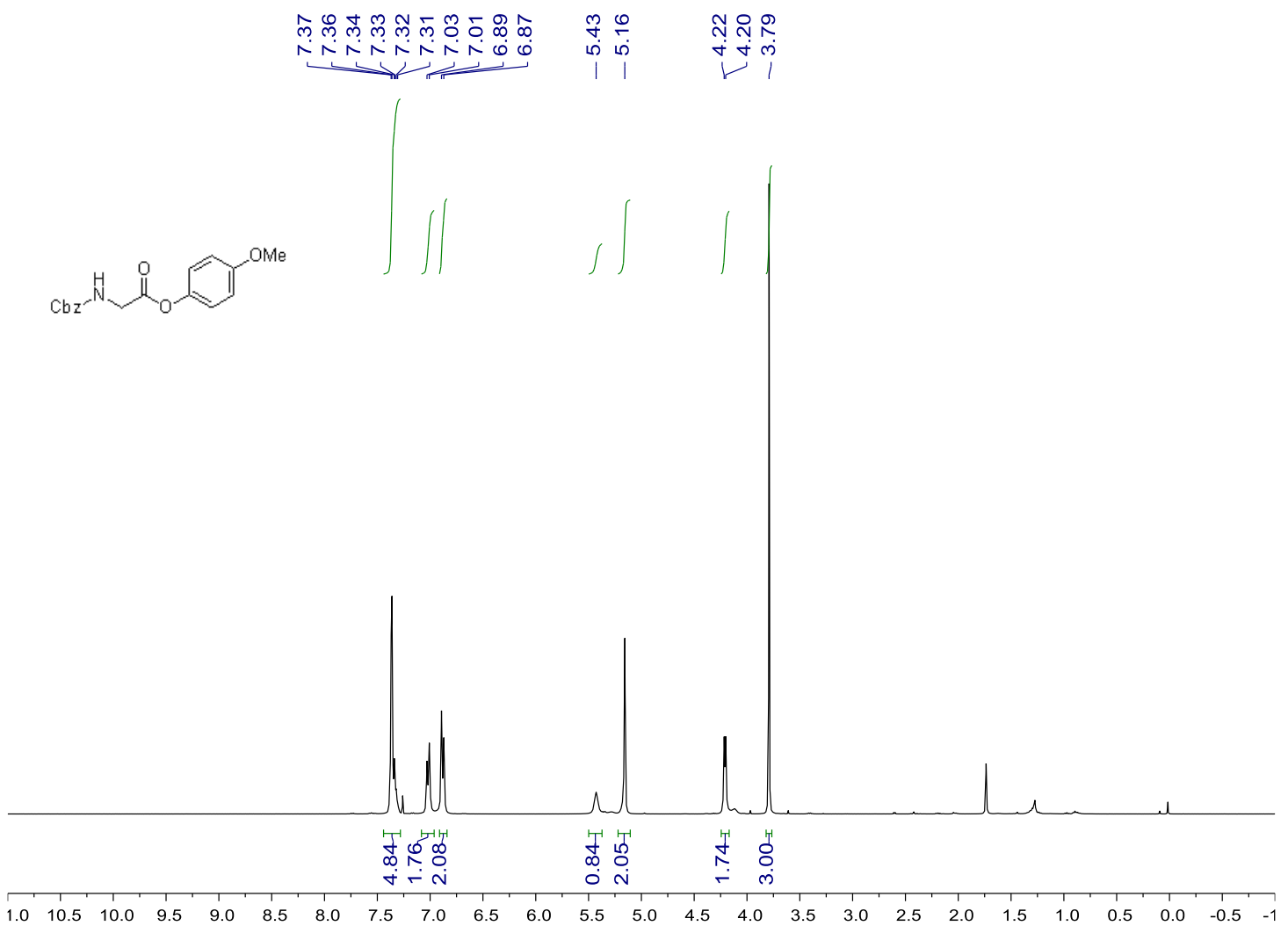

${ }^{13} \mathrm{C}\left\{{ }^{1} \mathrm{H}\right\}$-NMR $(100 \mathrm{MHz})$ spectrum of compound $\mathbf{6 g}$ in $\mathrm{CDCl}_{3}$
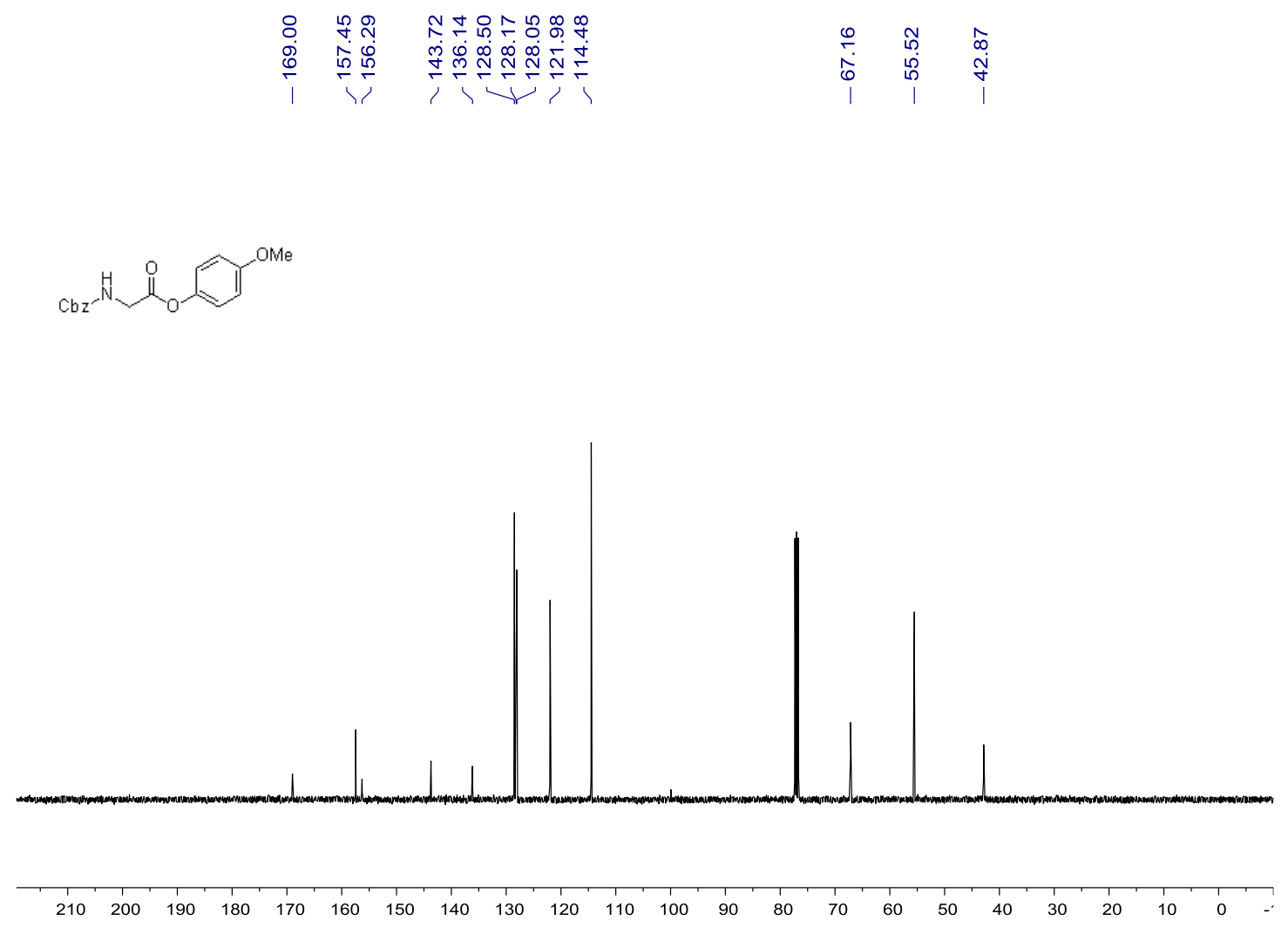

SI -40 
${ }^{1} \mathrm{H}-\mathrm{NMR}(400 \mathrm{MHz})$ spectrum of compound $\mathbf{6 h}$ in $\mathrm{CDCl}_{3}$

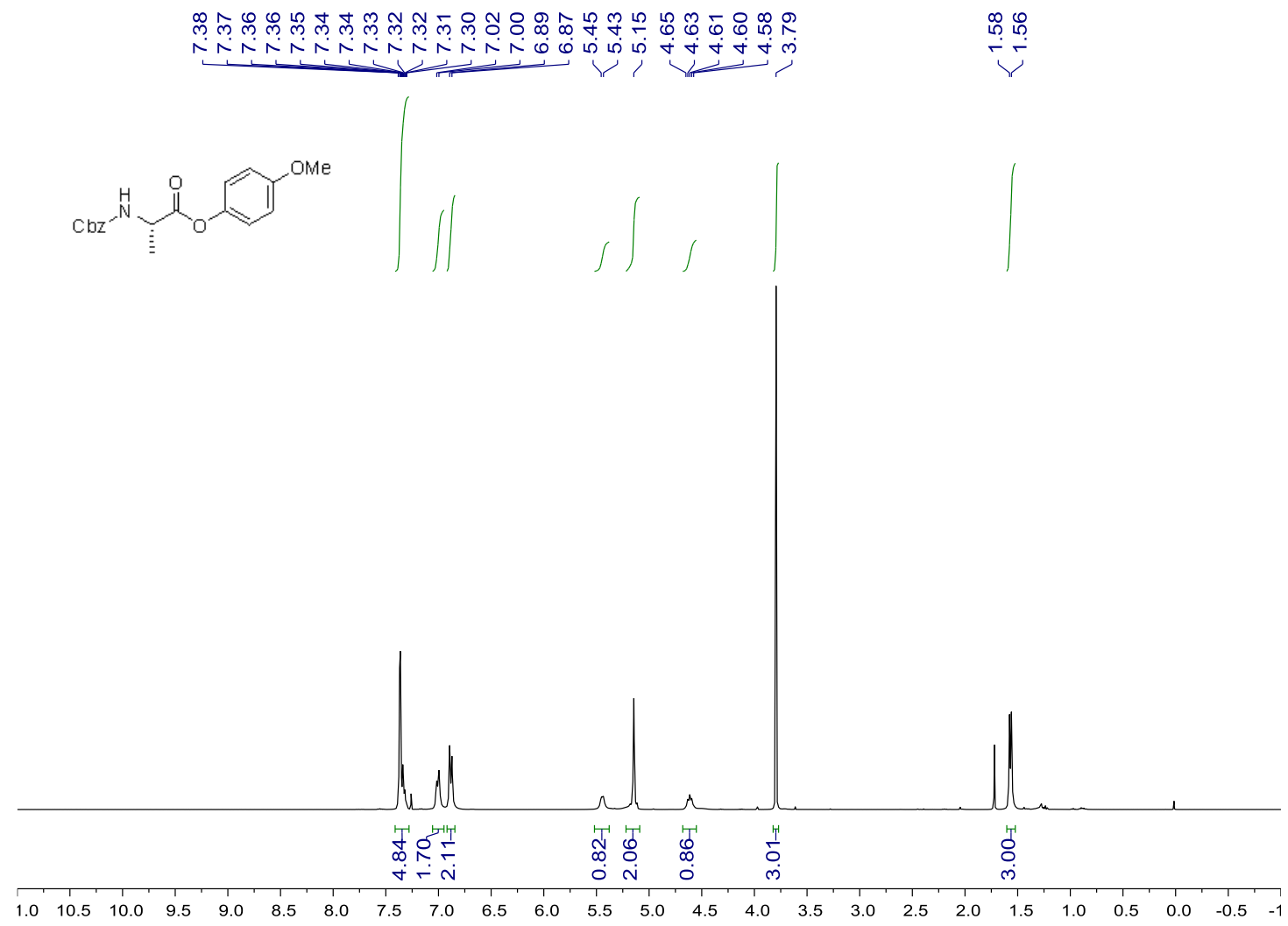

${ }^{13} \mathrm{C}\left\{{ }^{1} \mathrm{H}\right\}$-NMR (100 MHz) spectrum of compound $\mathbf{6 h}$ in $\mathrm{CDCl}_{3}$

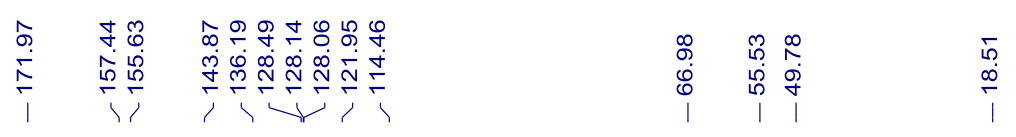<smiles>CCNC(C)C(=O)Oc1ccc(OC)cc1</smiles> 
${ }^{1} \mathrm{H}-\mathrm{NMR}\left(400 \mathrm{MHz}\right.$ ) spectrum of compound $\mathbf{6} \mathbf{i}$ in $\mathrm{CDCl}_{3}$

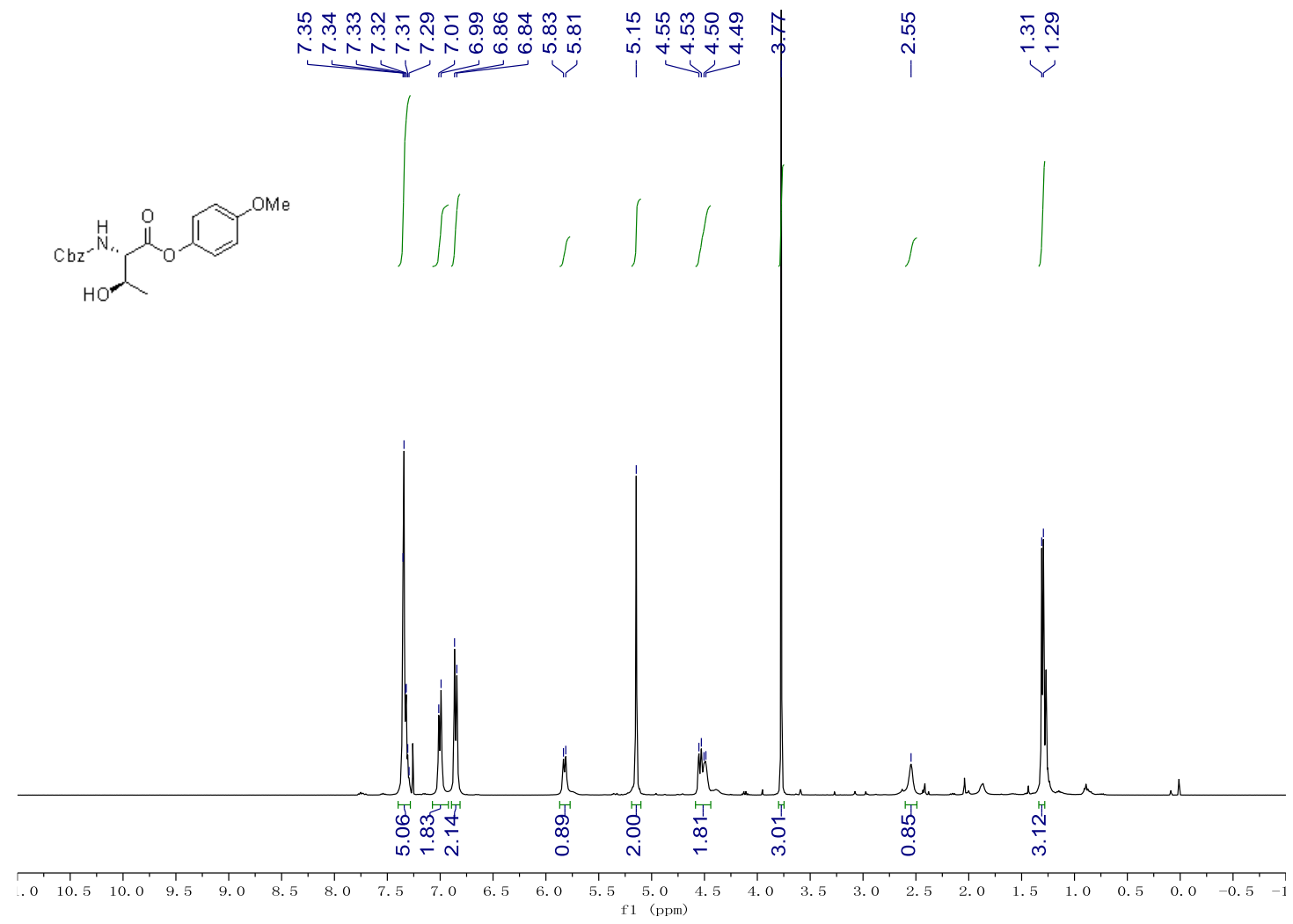

${ }^{13} \mathrm{C}\left\{{ }^{1} \mathrm{H}\right\}$-NMR (100 MHz) spectrum of compound $\mathbf{6 i}$ in $\mathrm{CDCl}_{3}$

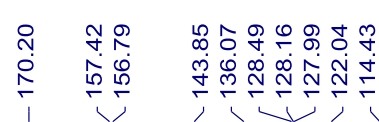

ț

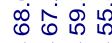

iे<smiles>CNC(C(=O)Oc1ccc(C)cc1)C(C)O</smiles>

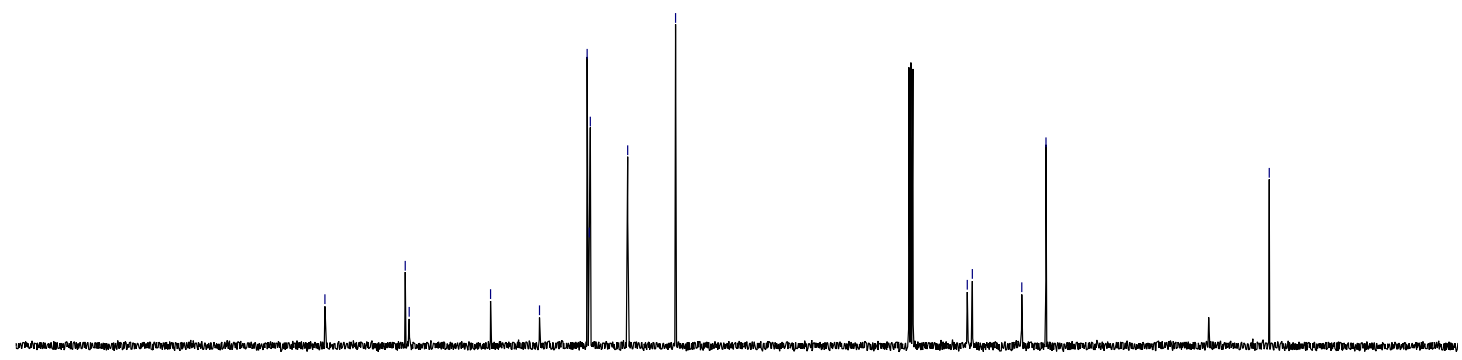

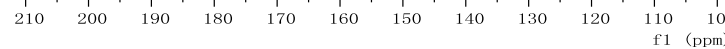


${ }^{1} \mathrm{H}-\mathrm{NMR}\left(400 \mathrm{MHz}\right.$ ) spectrum of compound $\mathbf{6 j}$ in $\mathrm{CDCl}_{3}$

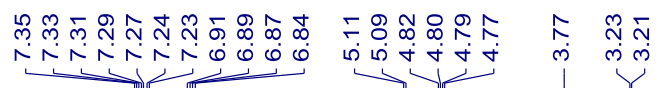

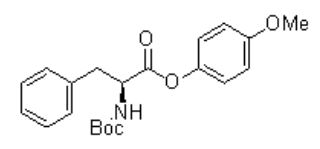

HI
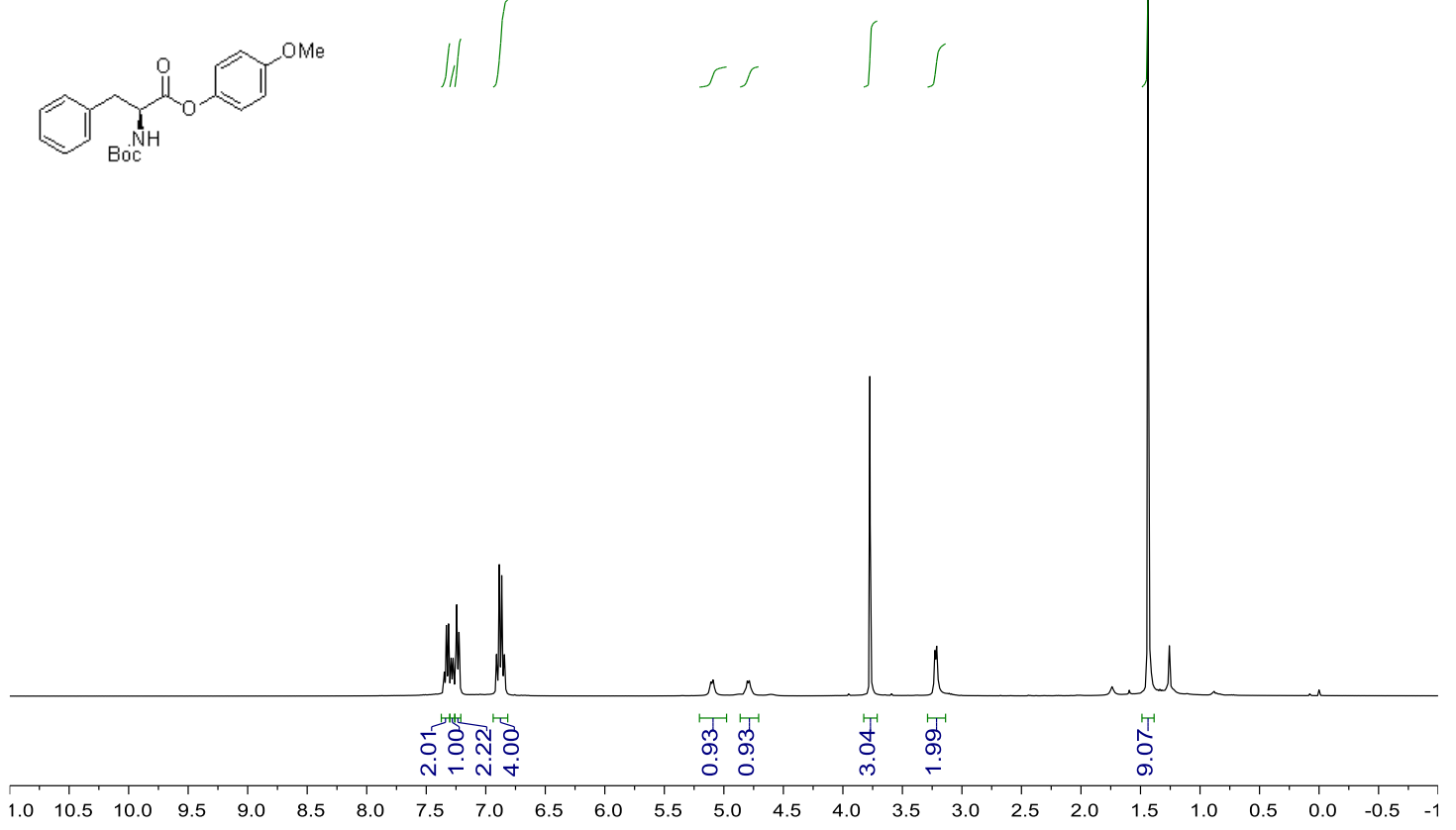

${ }^{13} \mathrm{C}\left\{{ }^{1} \mathrm{H}\right\}$-NMR (100 MHz) spectrum of compound $\mathbf{6 j}$ in $\mathrm{CDCl}_{3}$

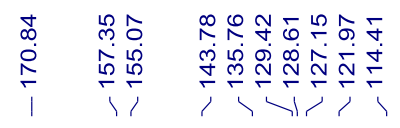

8
$\dot{0}$
0
1

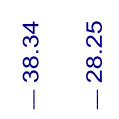
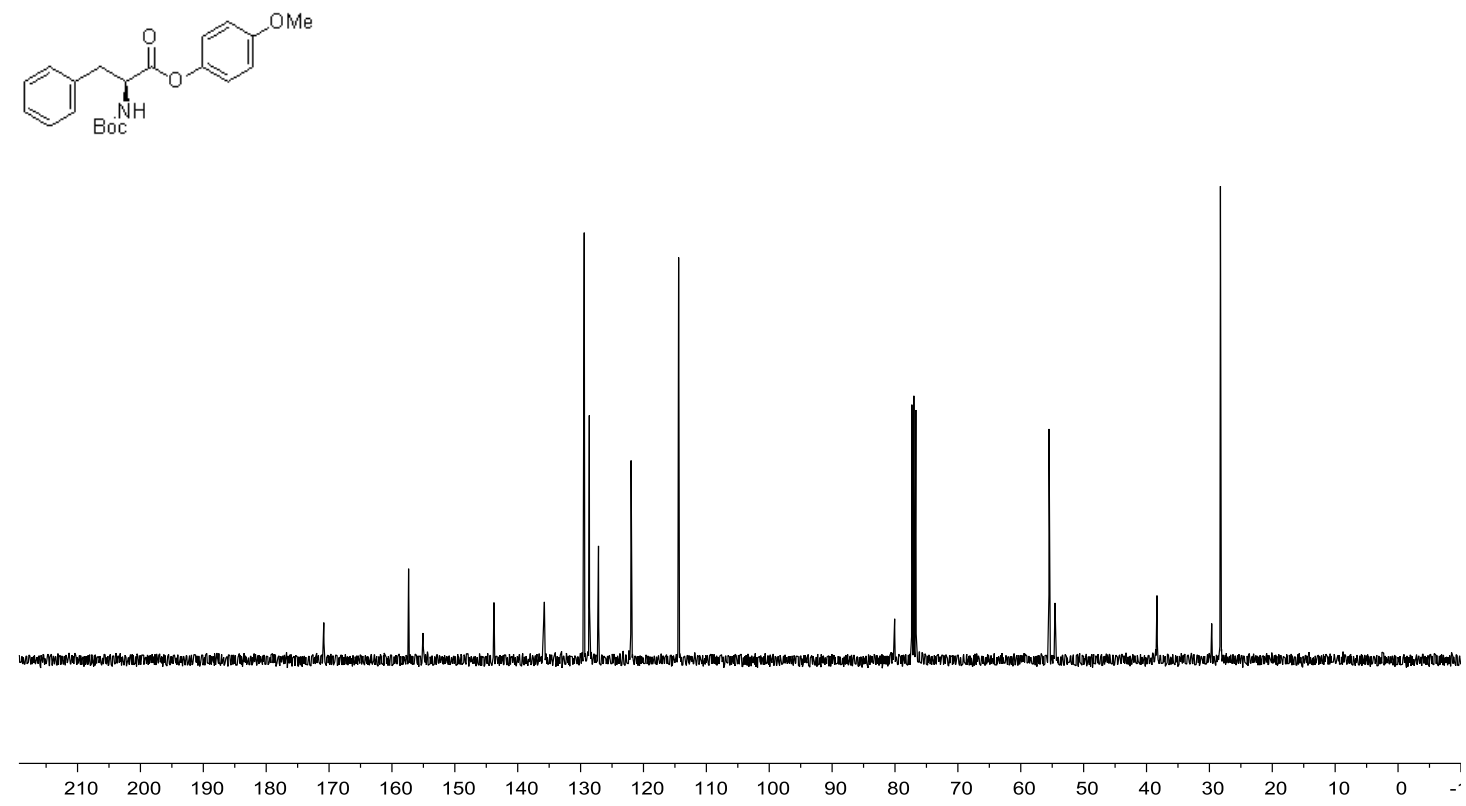

SI -43 
${ }^{1} \mathrm{H}-\mathrm{NMR}(400 \mathrm{MHz})$ spectrum of compound $\mathbf{6 k}$ in $\mathrm{CDCl}_{3}$

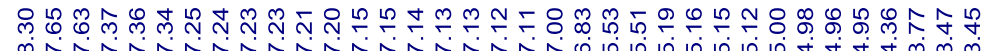

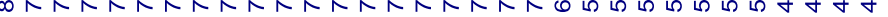
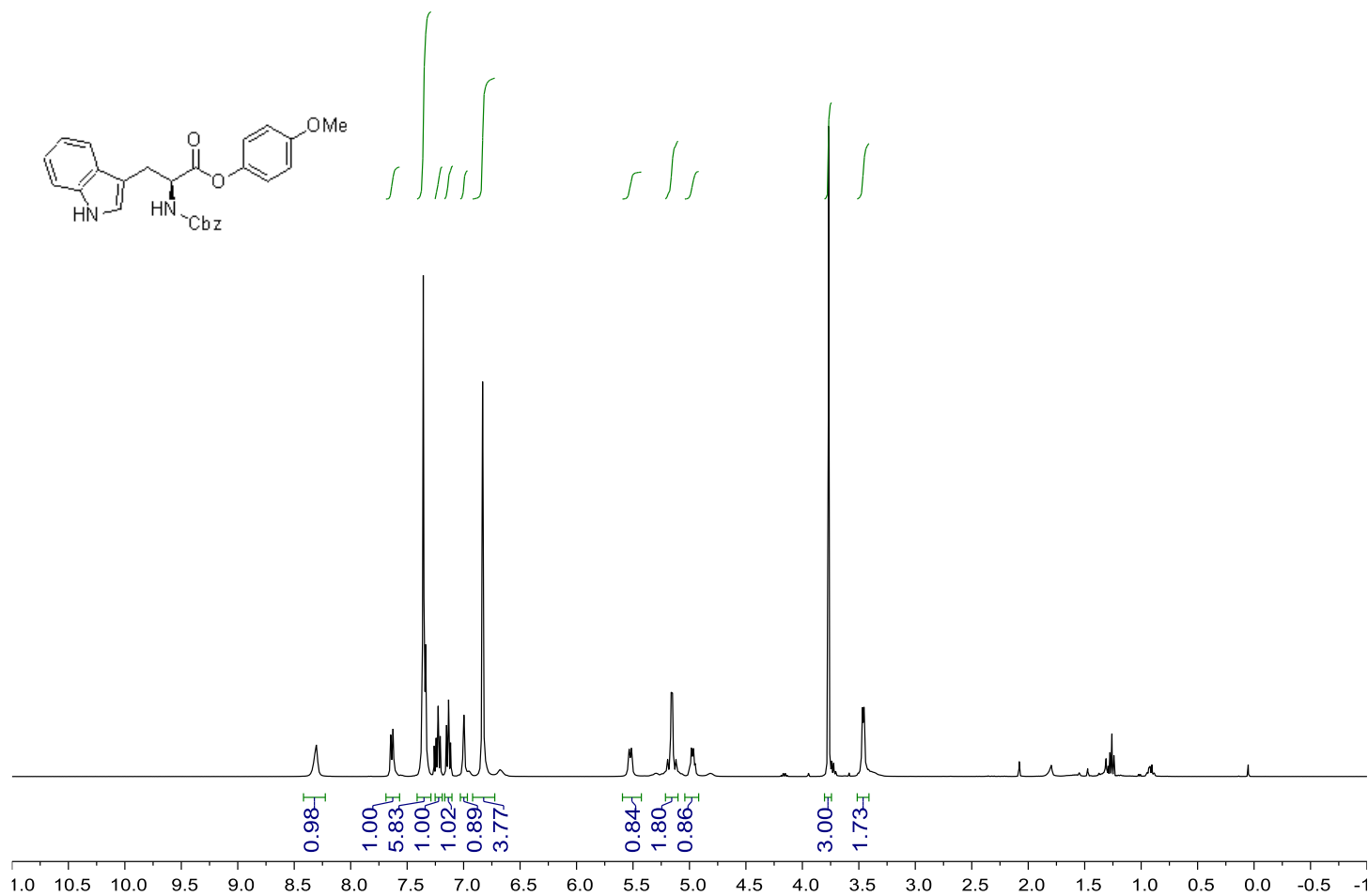

${ }^{13} \mathrm{C}\left\{{ }^{1} \mathrm{H}\right\}-\mathrm{NMR}(100 \mathrm{MHz})$ spectrum of compound $\mathbf{6} \mathbf{k}$ in $\mathrm{CDCl}_{3}$

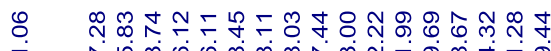

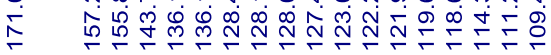

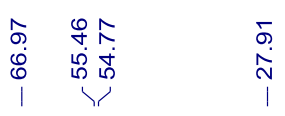
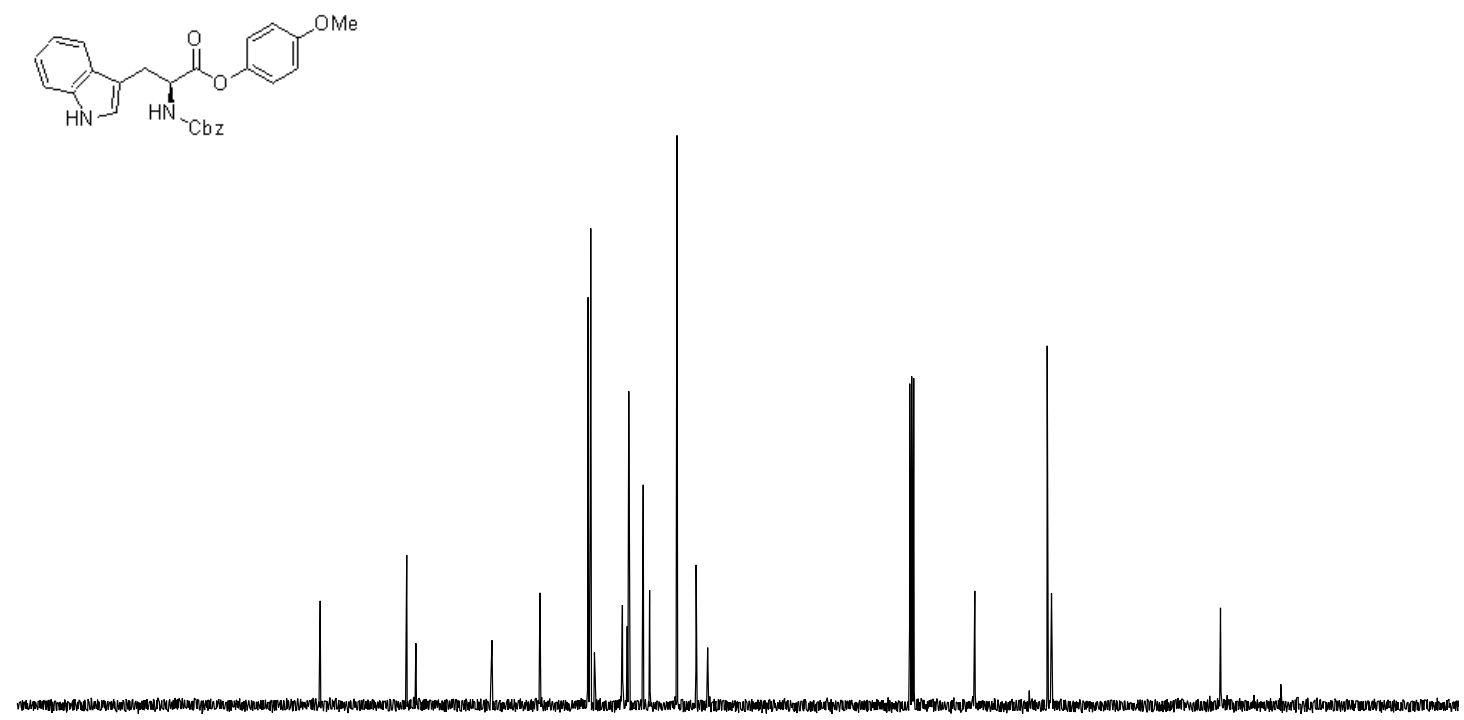

$\begin{array}{llllllllllllllllllllll}210 & 200 & 190 & 180 & 170 & 160 & 150 & 140 & 130 & 120 & 110 & 100 & 90 & 80 & 70 & 60 & 50 & 40 & 30 & 20 & 10 & 0\end{array}$ 
${ }^{1} \mathrm{H}-\mathrm{NMR}\left(400 \mathrm{MHz}\right.$ ) spectrum of compound $\mathbf{6 l}$ in $\mathrm{CDCl}_{3}$

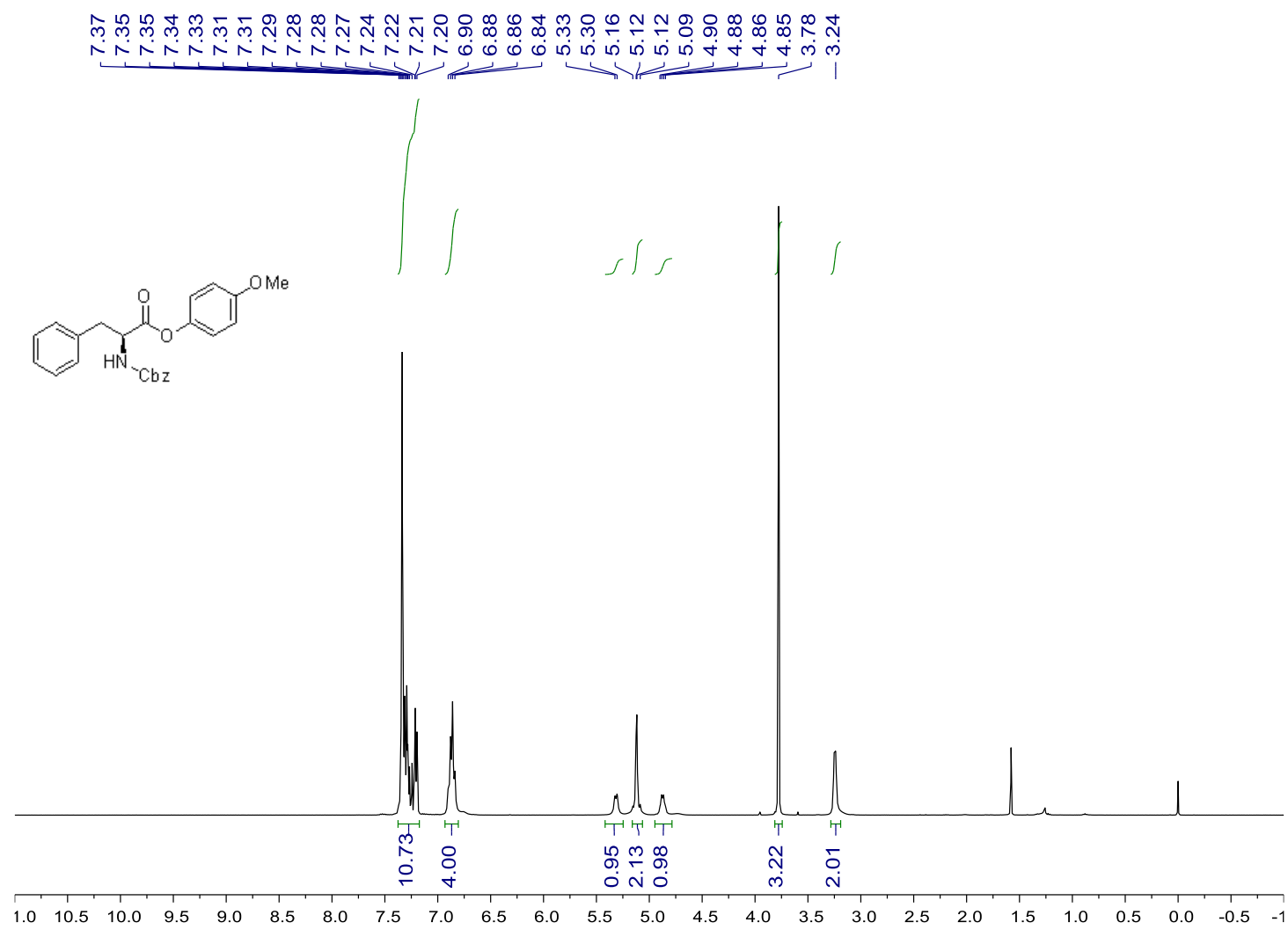

${ }^{13} \mathrm{C}\left\{{ }^{1} \mathrm{H}\right\}$-NMR (100 MHz) spectrum of compound $\mathbf{6} \mathbf{~ i n ~} \mathrm{CDCl}_{3}$
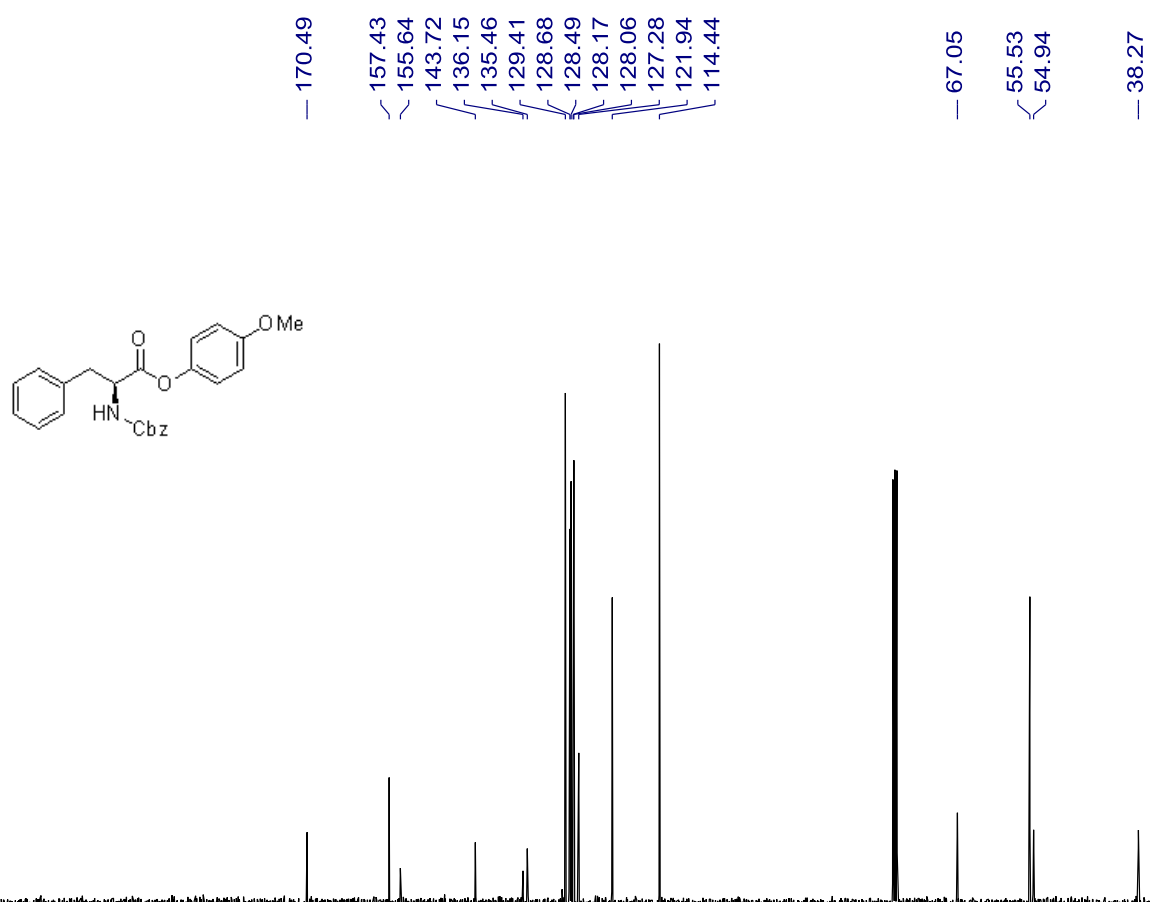


\section{HPLC data}

HPLC profile of racemate of $\mathbf{6 h}$

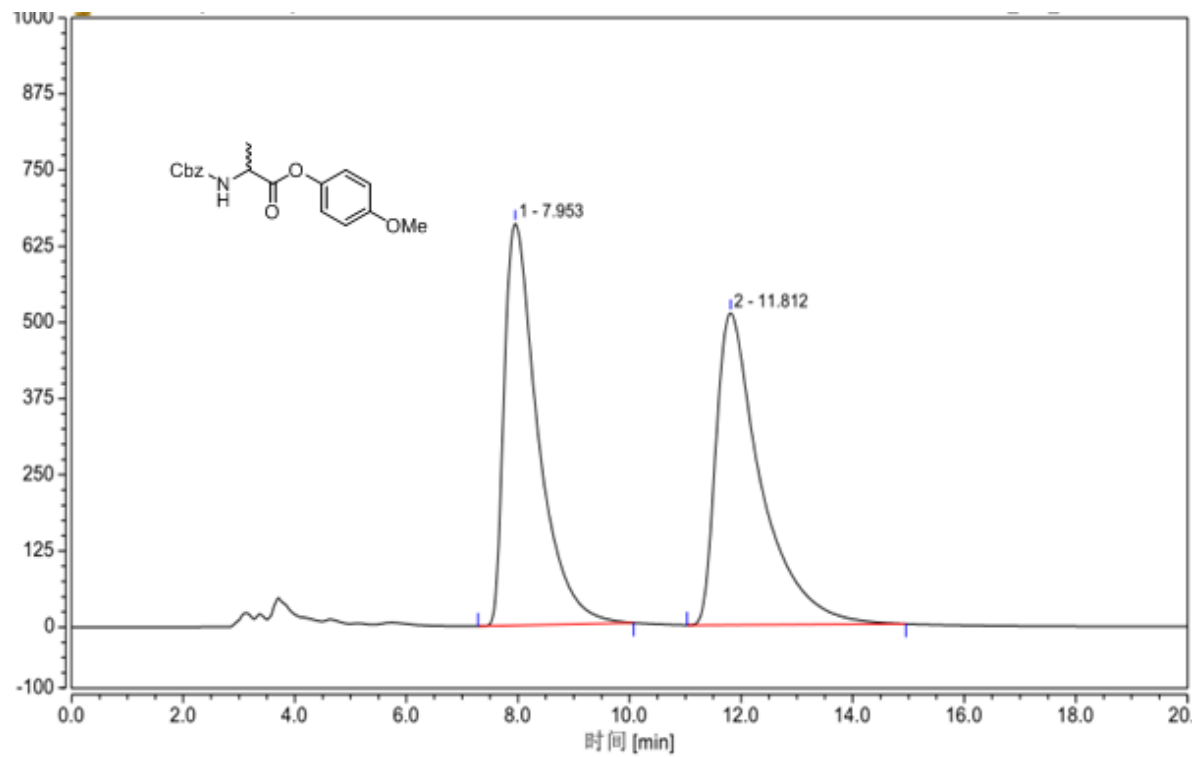

Peak Info

\begin{tabular}{|c|c|c|c|c|}
\hline Peak No & \%Area & Area & RT ( min ) & Height(mAv) \\
\hline 1 & 49.18 & 460.944 & 7.953 & 659.527 \\
\hline 2 & 50.82 & 476.402 & 11.812 & 511.958 \\
\hline Total: & 100 & 937.346 & & 1171.485 \\
\hline
\end{tabular}

HPLC profile of compound $\mathbf{6 h}$ by using $\mathrm{NEt}_{3}$ as the catalyst

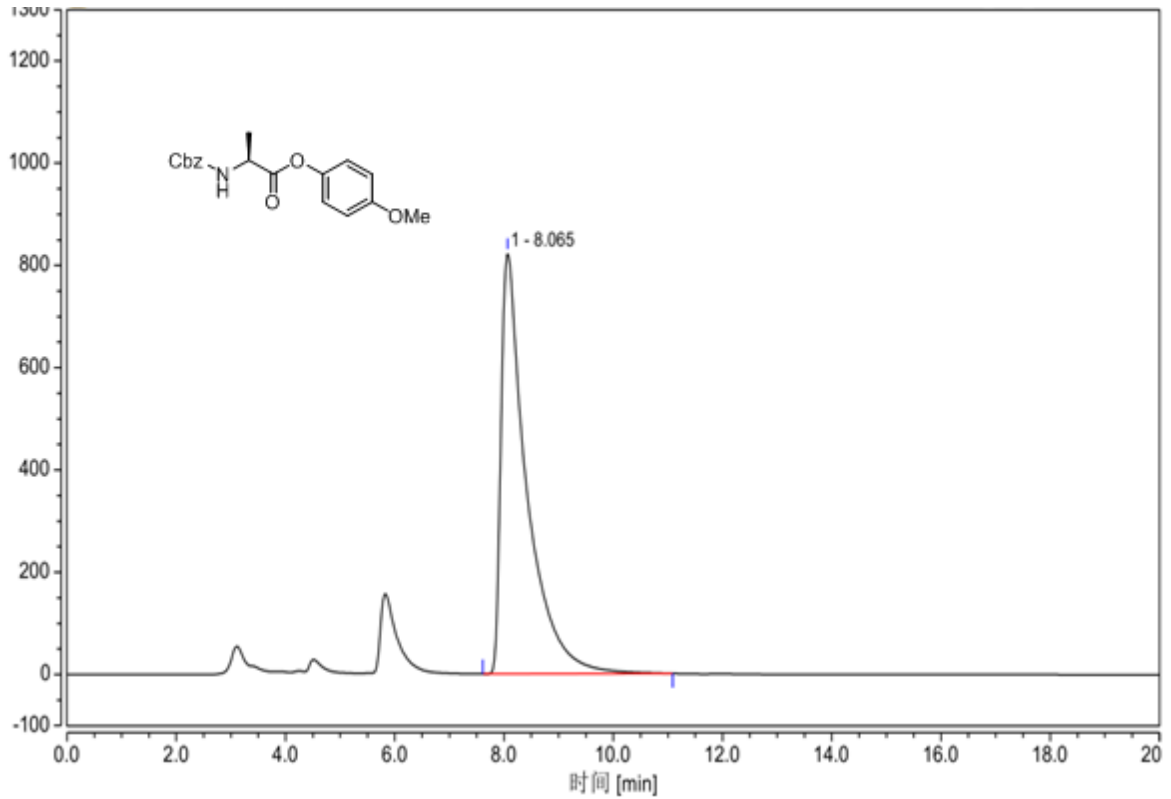

Peak Info

\begin{tabular}{|c|c|c|c|c|}
\hline Peak No & \%Area & Area & RT $(\min )$ & Height $(\mathrm{mAv})$ \\
\hline 1 & 100 & 448.000 & 8.065 & 823.544 \\
\hline Total: & 100 & 448.000 & & 823.544 \\
\hline
\end{tabular}


HPLC profile of the racemate of $\mathbf{6} \mathbf{i}$

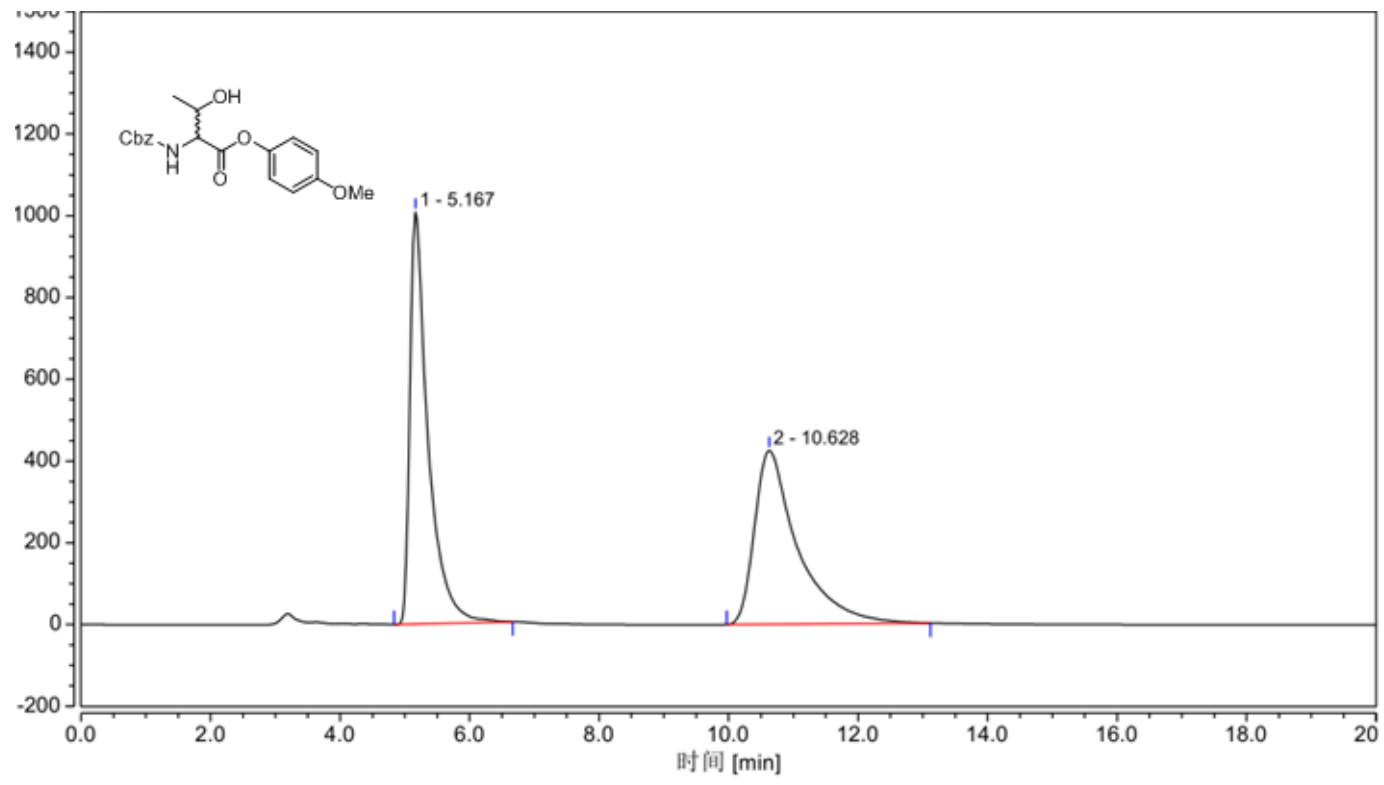

Peak Info

\begin{tabular}{|c|c|c|c|c|}
\hline Peak No & \%Area & Area & RT $(\min )$ & Height(mAv) \\
\hline 1 & 50.08 & 327.420 & 5.167 & 1007.364 \\
\hline 2 & 49.92 & 326.378 & 10.628 & 425.031 \\
\hline Total: & 100 & 653.798 & & 1432.395 \\
\hline
\end{tabular}

HPLC profile of compound $\mathbf{6} \mathbf{i}$ by using $\mathrm{NEt}_{3}$ as the catalyst

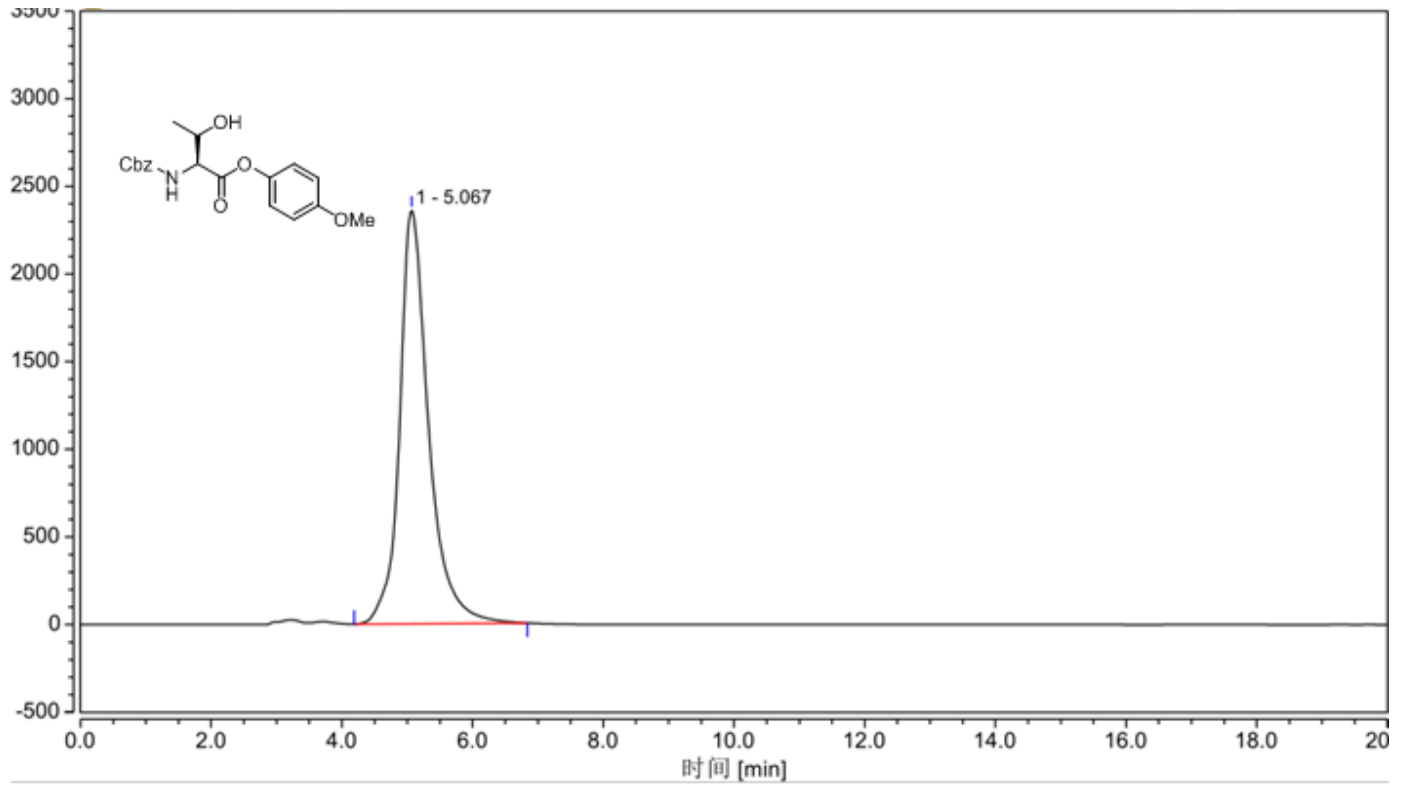

Peak Info

\begin{tabular}{|c|c|c|c|c|}
\hline Peak No & \%Area & Area & RT $(\min )$ & Height $(\mathrm{mAv})$ \\
\hline 1 & 100 & 1213.385 & 5.067 & 2359.758 \\
\hline Total: & 100 & 1213.385 & & 2359.758 \\
\hline
\end{tabular}


HPLC profile of the racemate of $\mathbf{6 j}$

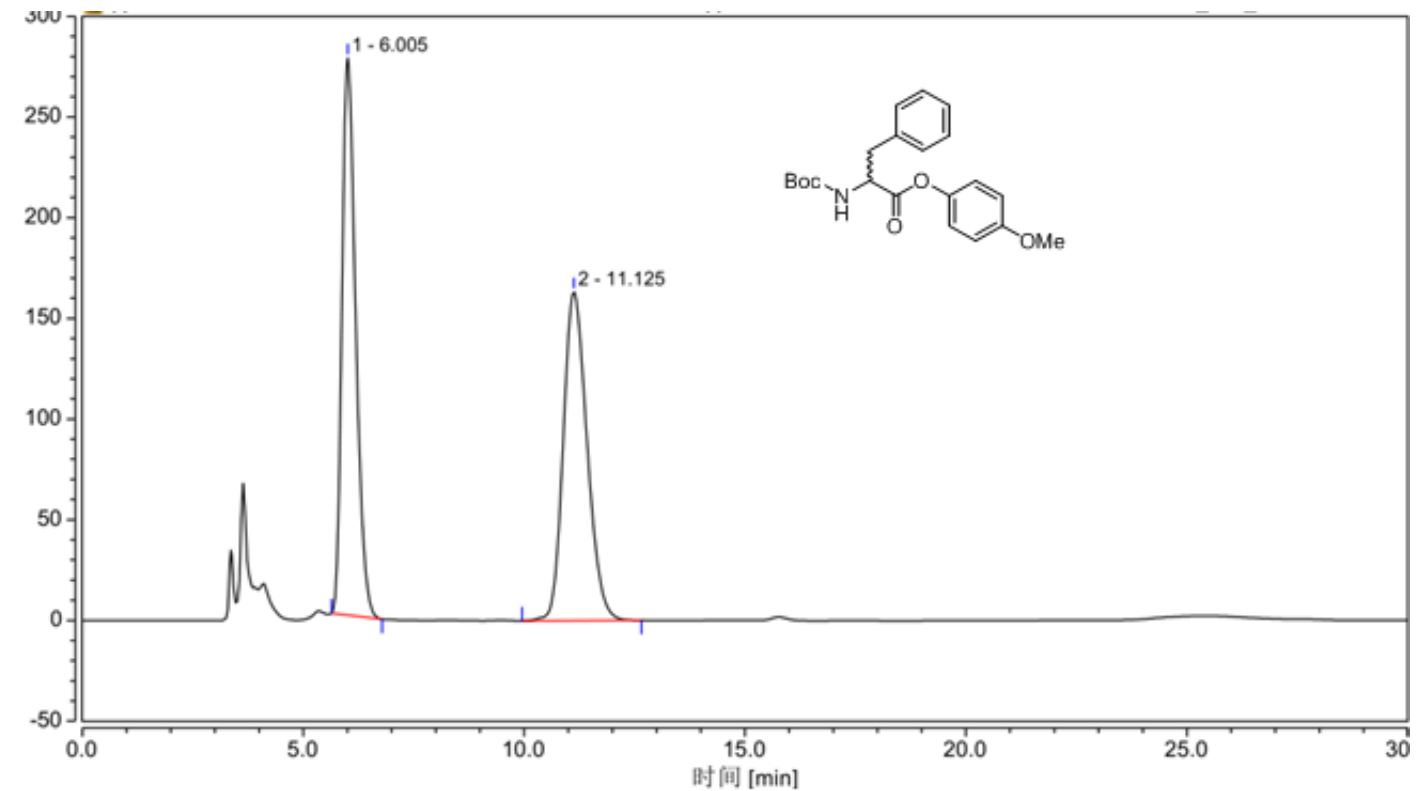

Peak Info

\begin{tabular}{|c|c|c|c|c|}
\hline Peak No & \%Area & Area & RT $(\min )$ & Height $(\mathrm{mAv})$ \\
\hline 1 & 49.37 & 99.720 & 6.005 & 276.759 \\
\hline 2 & 50.63 & 102.261 & 11.125 & 163.386 \\
\hline Total: & 100 & 201.981 & & 440.145 \\
\hline
\end{tabular}

HPLC profile of compound $\mathbf{6 j}$ by using $\mathrm{NEt}_{3}$ as the catalyst

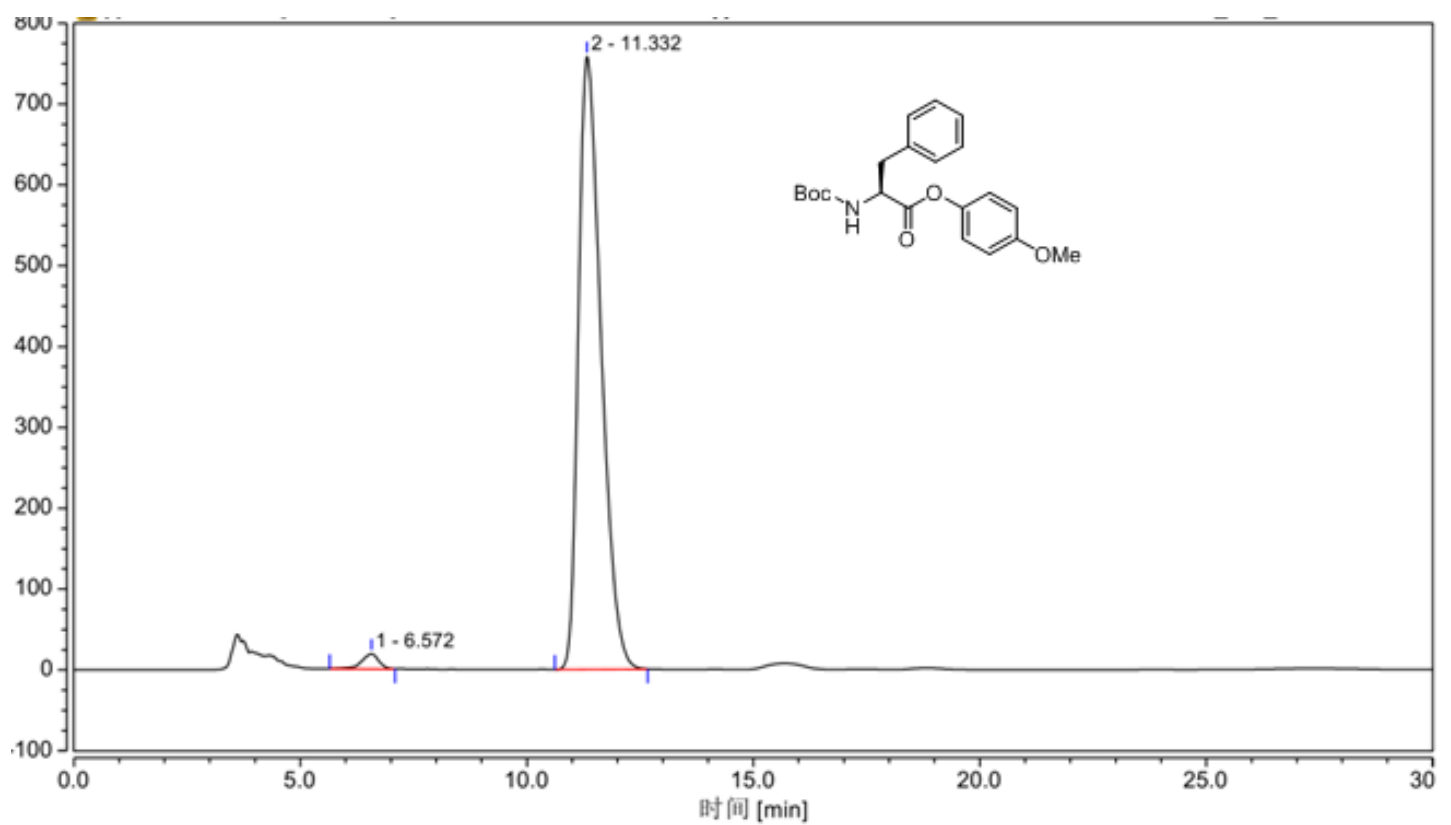

Peak Info

\begin{tabular}{|c|c|c|c|c|}
\hline Peak No & \%Area & Area & RT $($ min $)$ & Height(mAv) \\
\hline 1 & 1.86 & 8.367 & 6.572 & 18.953 \\
\hline 2 & 98.14 & 440.883 & 11.332 & 758.77 \\
\hline Total: & 100 & 449.251 & & 777.130 \\
\hline
\end{tabular}


HPLC profile of compound $\mathbf{6 j}$ by using N,N-Diisopropylethylamine (DIEA) as the catalyst

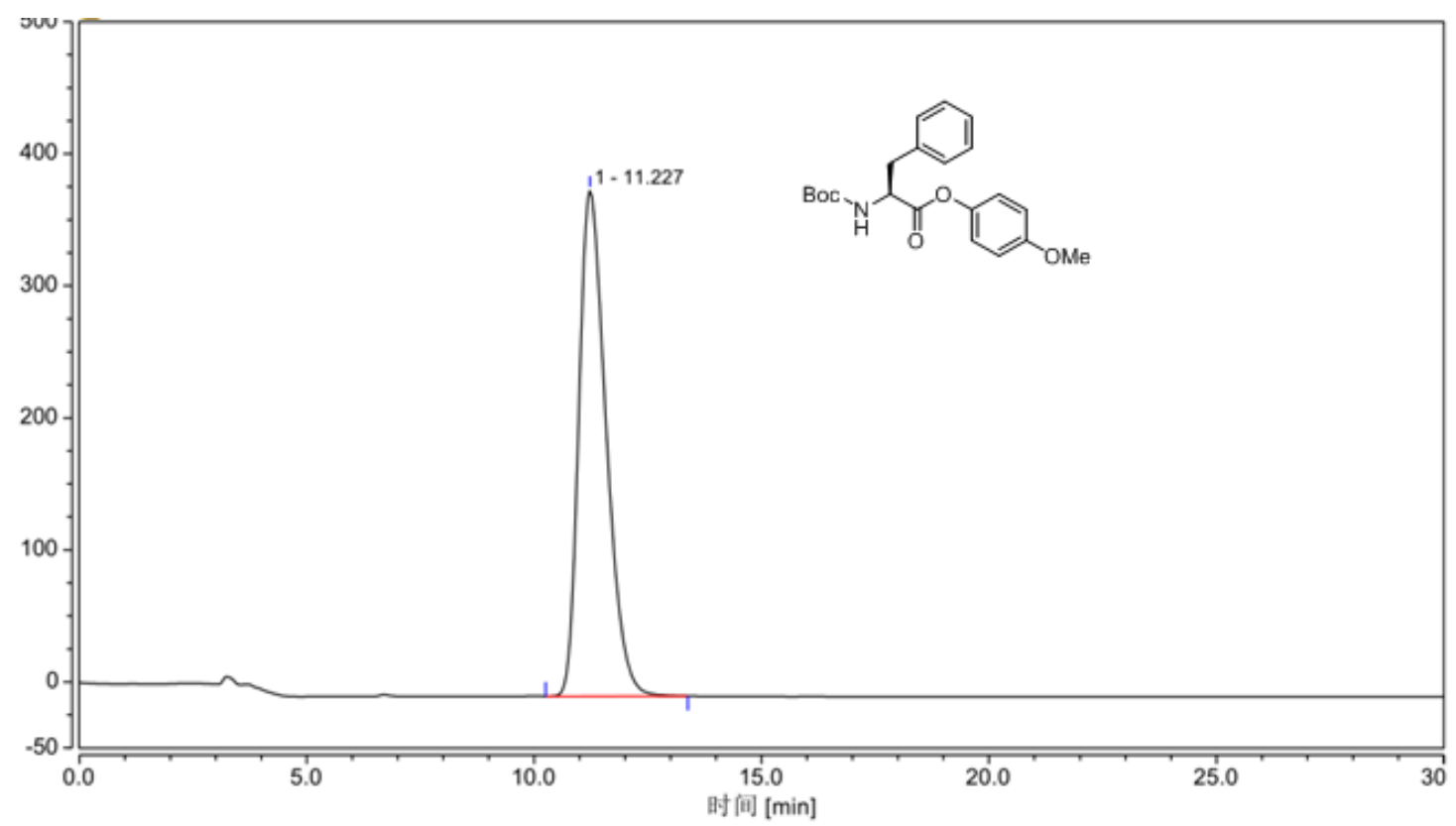

Peak Info

\begin{tabular}{|c|c|c|c|c|}
\hline Peak No & \%Area & Area & RT $(\min )$ & Height $(\mathrm{mAv})$ \\
\hline 1 & 100 & 265.868 & 11.227 & 382.923 \\
\hline Total: & 100 & 265.868 & - & 382.923 \\
\hline
\end{tabular}

HPLC profile of racemate of $\mathbf{6 k}$

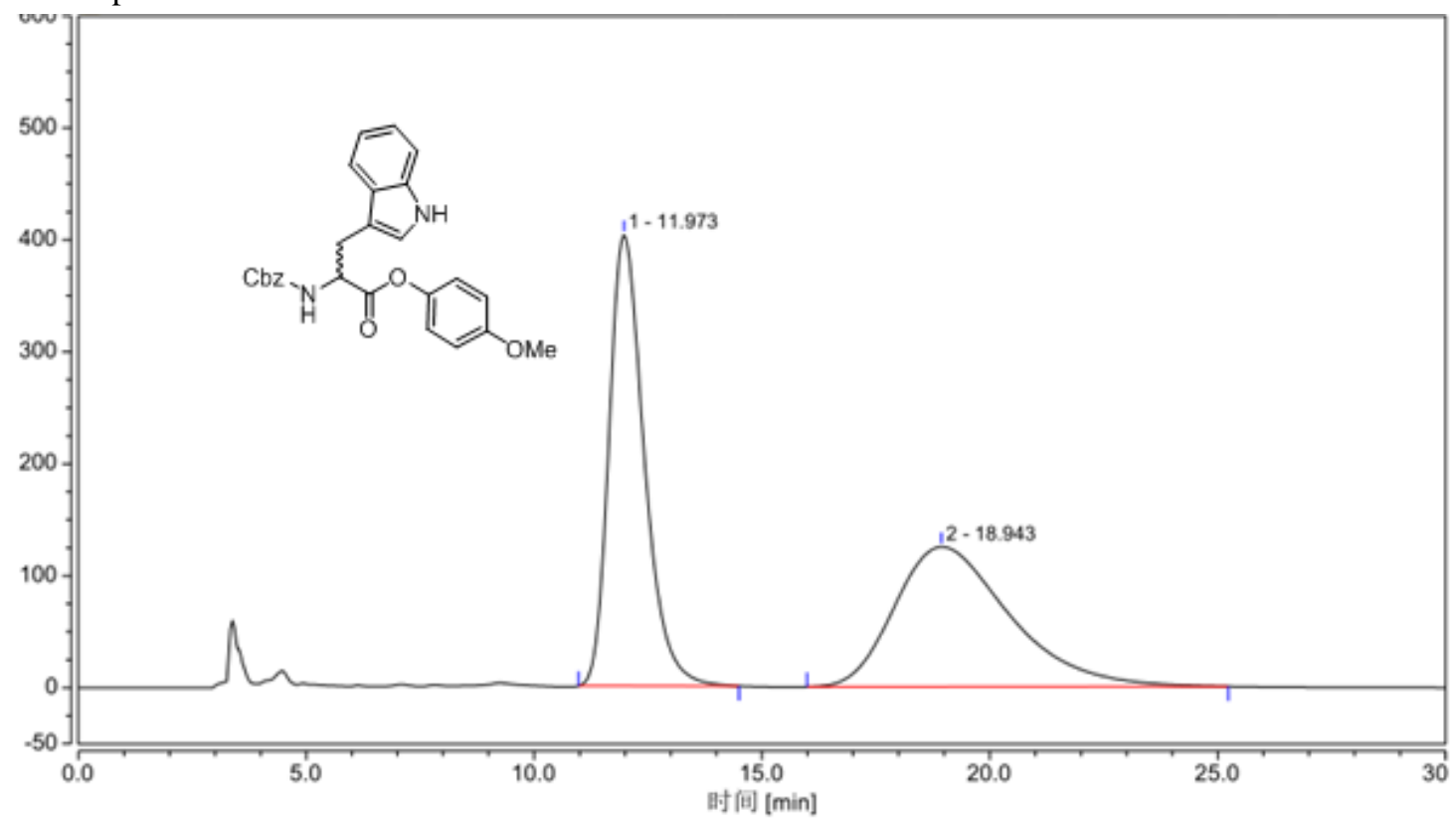

Peak Info

\begin{tabular}{|c|c|c|c|c|}
\hline Peak No & \%Area & Area & RT $(\mathrm{min})$ & Height(mAv) \\
\hline 1 & 49.61 & 360.452 & 11.973 & 402.818 \\
\hline 2 & 50.39 & 366.062 & 18.943 & 124.929 \\
\hline Total: & 100 & 726.514 & & 527.747 \\
\hline
\end{tabular}


HPLC profile of compound $\mathbf{6 k}$ by using $\mathrm{NEt}_{3}$ as the catalyst

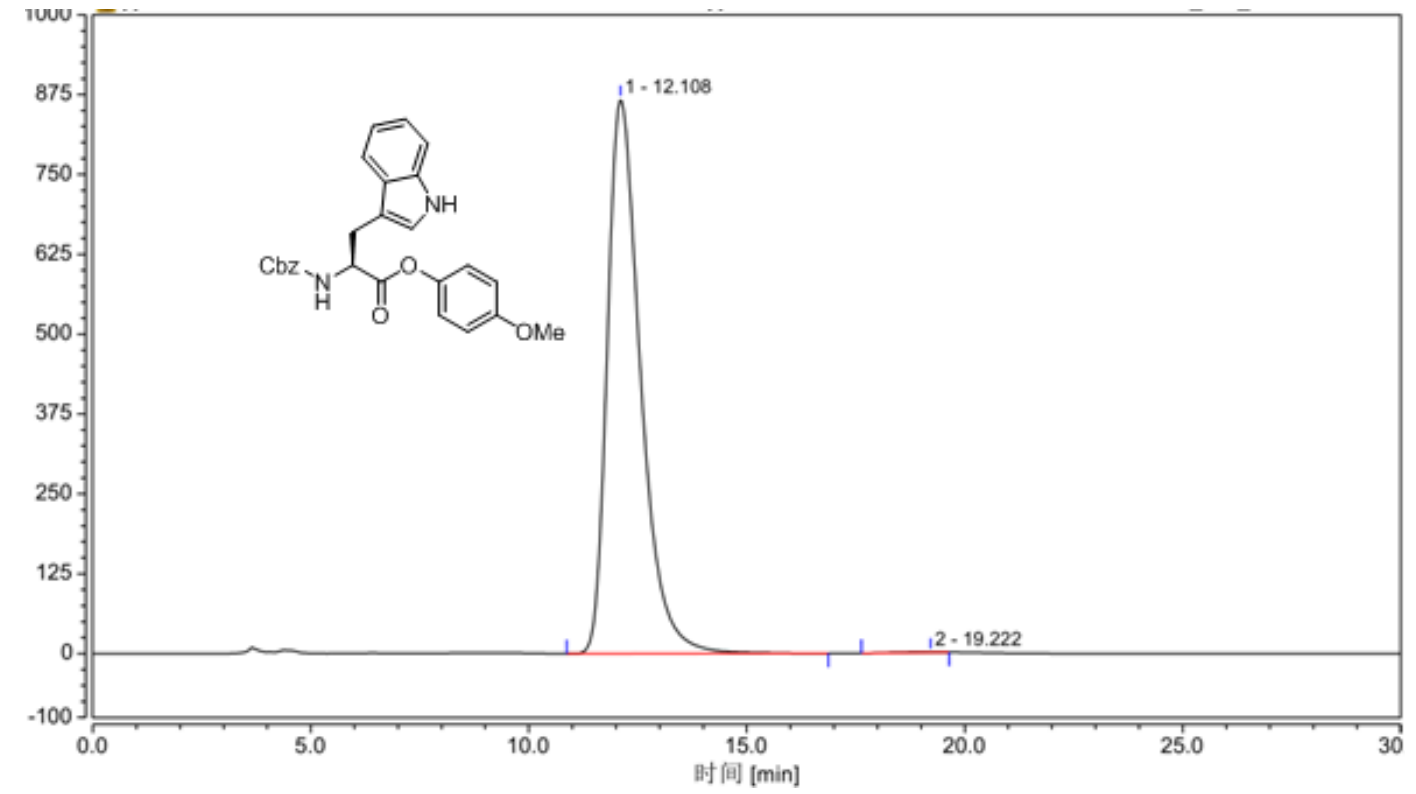

Peak Info

\begin{tabular}{|c|c|c|c|c|}
\hline Peak No & \%Area & Area & RT $(\mathrm{min})$ & Height(mAv) \\
\hline 1 & 99.91 & 767.395 & 12.108 & 867.347 \\
\hline 2 & 0.09 & 0.676 & 19.222 & 0.477 \\
\hline Total: & 100 & 768.071 & & 867.824 \\
\hline
\end{tabular}

HPLC profile of compound $\mathbf{6 k}$ by using N,N-Diisopropylethylamine (DIEA) as the catalyst

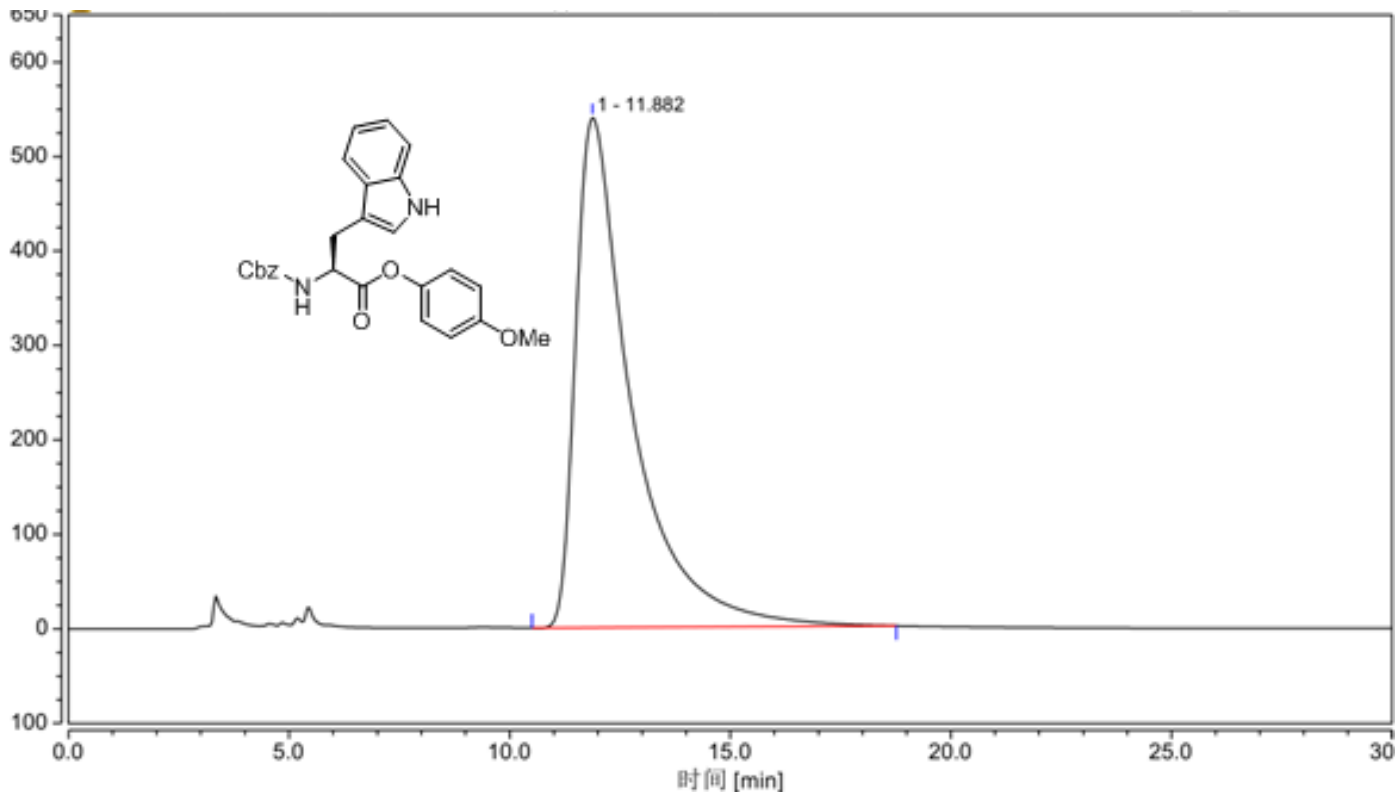

Peak Info

\begin{tabular}{|c|c|c|c|c|}
\hline Peak No & \%Area & Area & RT $(\min )$ & Height(mAv) \\
\hline 1 & 100 & 792.898 & 11.882 & 540.030 \\
\hline Total: & 100 & 792.898 & - & 540.030 \\
\hline
\end{tabular}




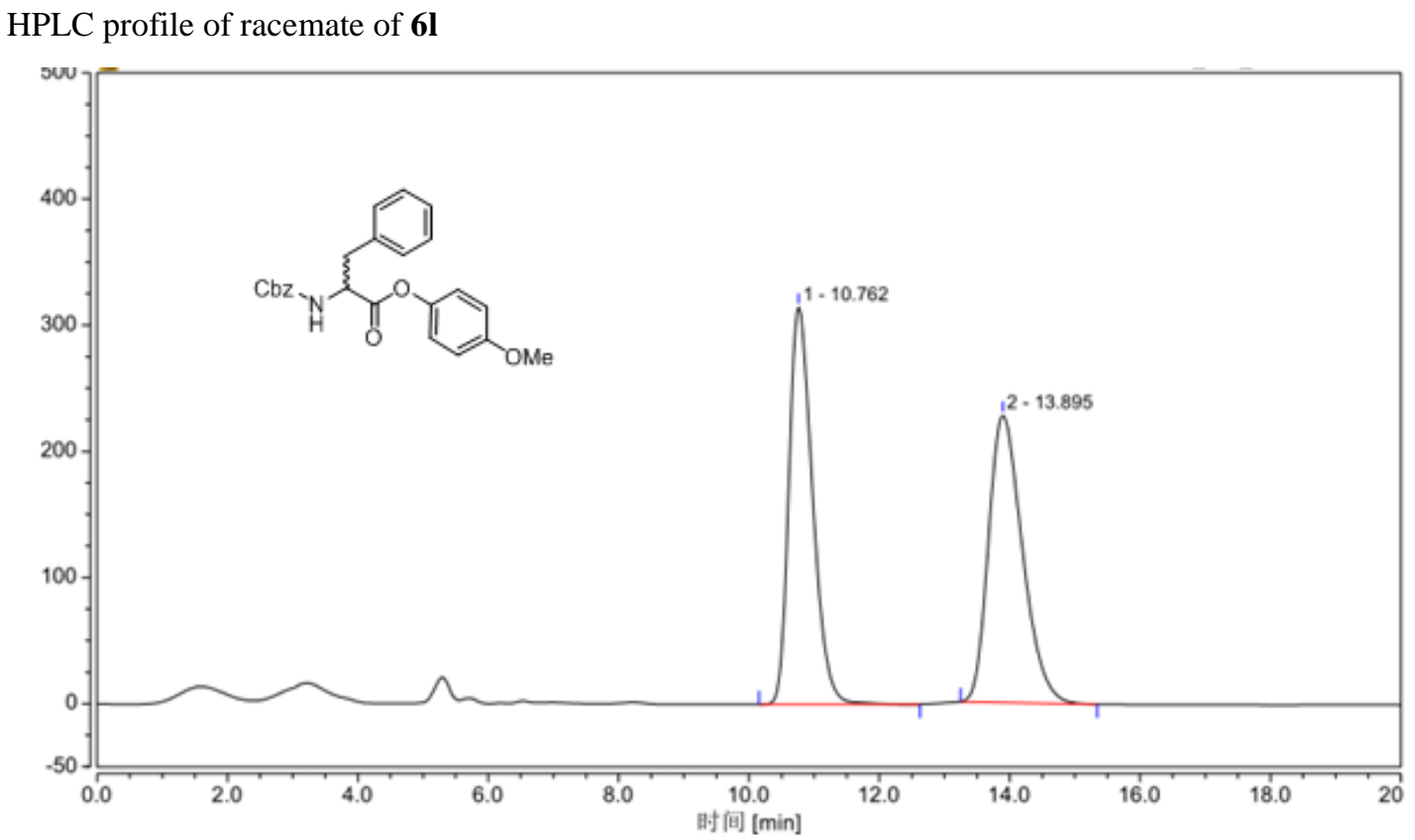

Peak Info

\begin{tabular}{|c|c|c|c|c|}
\hline Peak No & \%Area & Area & RT $(\min )$ & Height(mAv) \\
\hline 1 & 49.09 & 132.348 & 10.762 & 314.969 \\
\hline 2 & 50.91 & 137.242 & 13.895 & 227.671 \\
\hline Total: & 100 & 269.590 & & 542.641 \\
\hline
\end{tabular}

HPLC profile of compound $\mathbf{6}$ by using $\mathrm{NEt}_{3}$ as the catalyst

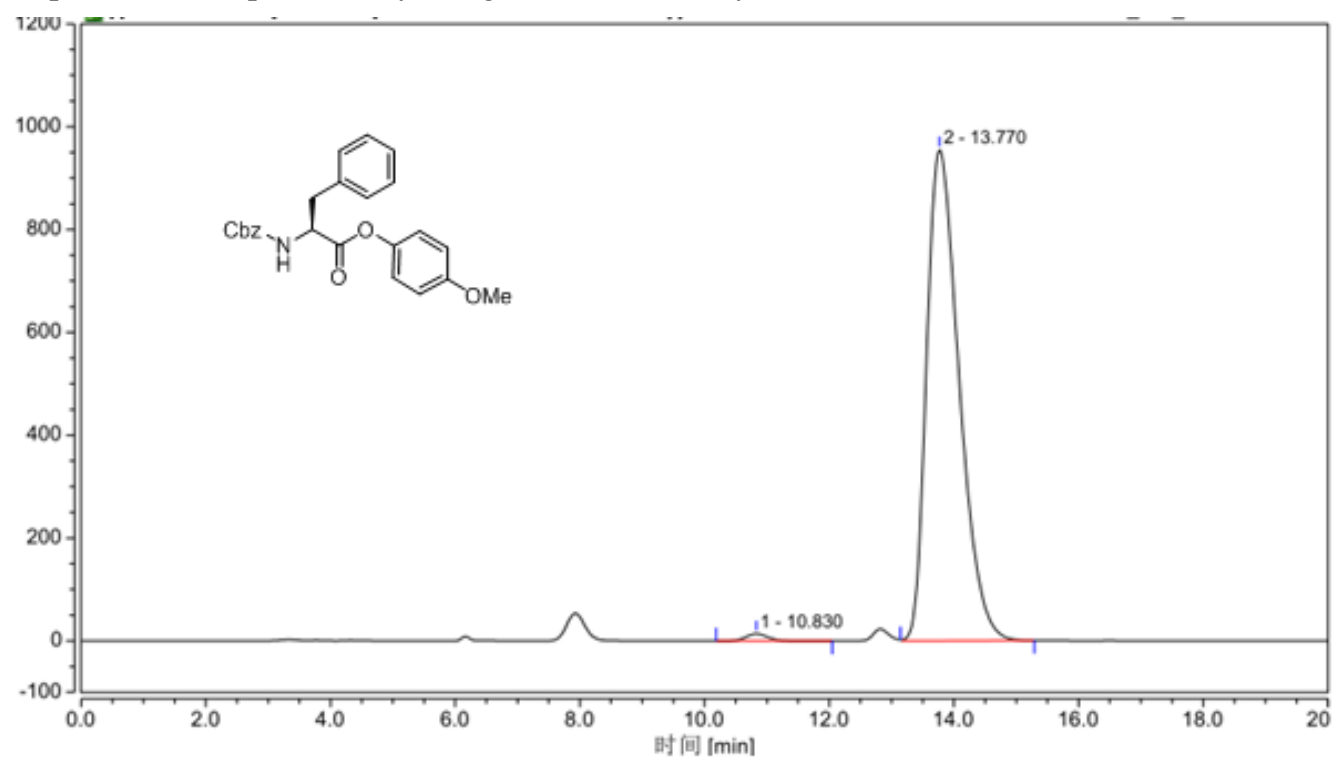

Peak Info

\begin{tabular}{|c|c|c|c|c|}
\hline Peak No & \%Area & Area & RT $(\min )$ & Height $(\mathrm{mAv})$ \\
\hline 1 & 0.98 & 5.709 & 10.830 & 13.612 \\
\hline 2 & 99.02 & 576.568 & 13.770 & 955.237 \\
\hline Total: & 100 & 582.277 & & 968.849 \\
\hline
\end{tabular}

SI-51 
HPLC profile of compound $\mathbf{6} \mathbf{l}$ by using N,N-Diisopropylethylamine (DIEA) as the catalyst

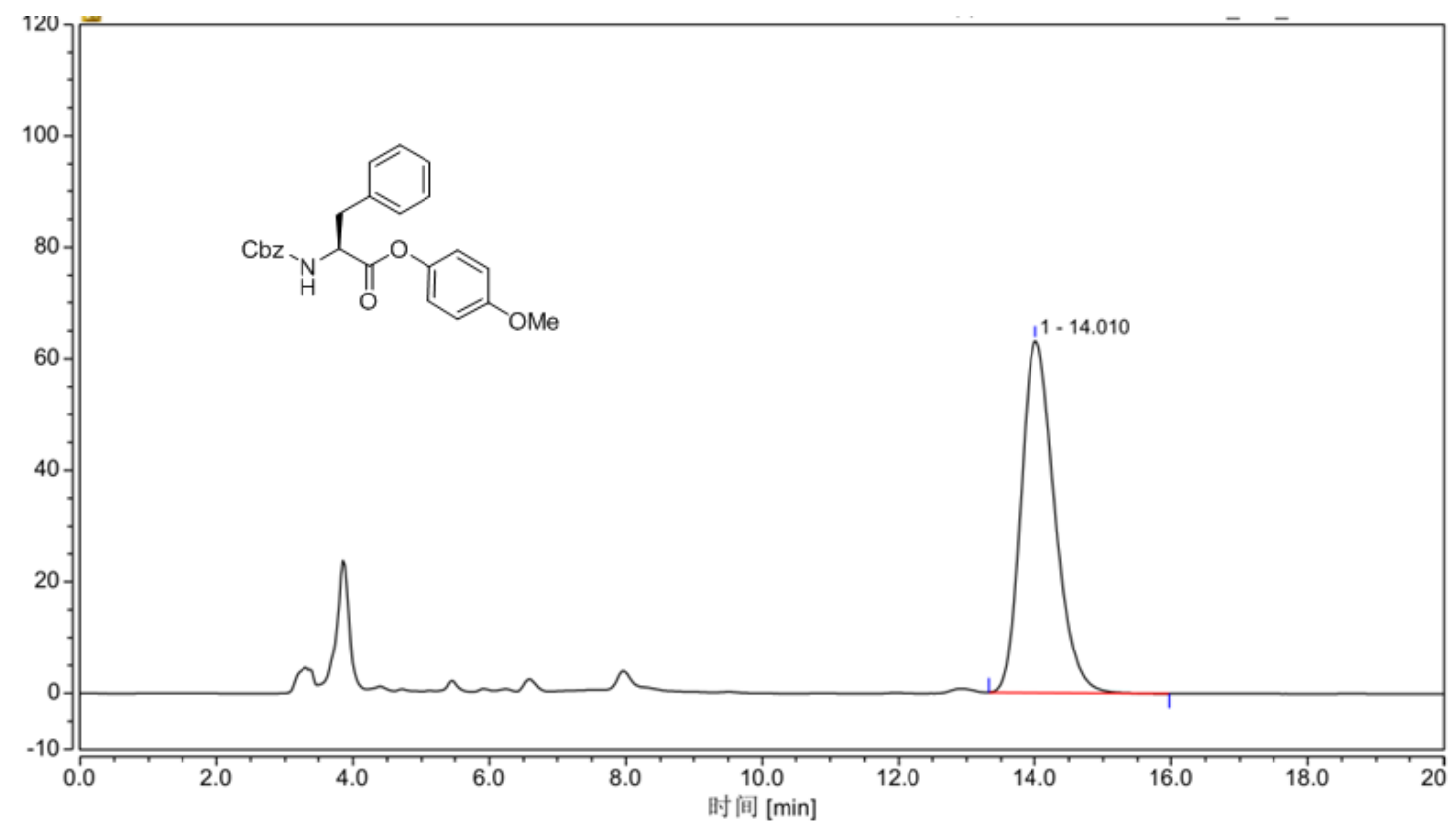

Peak Info

\begin{tabular}{|c|c|c|c|c|}
\hline Peak No & \%Area & Area & RT $(\min )$ & Height $(\mathrm{mAv})$ \\
\hline 1 & 100 & 36.425 & 14.010 & 63.185 \\
\hline Total: & 100 & 36.425 & & 63.185 \\
\hline
\end{tabular}

